\title{
Investigation of the Relation between the Spectral Energy Distributions and the Emission Lines in Low\#Redshift Quasars
}

\section{Citation}

Wilkes, Belinda J., Joanna Kuraszkiewicz, Paul J. Green, Smita Mathur, and Jonathan C. McDowell. 1999. "Investigation of the Relation Between the Spectral Energy Distributions and the Emission Lines in Low\#Redshift Quasars." The Astrophysical Journal 513 (1) (March): 76107. doi:10.1086/306828.

\section{Published Version}

doi:10.1086/306828

\section{Permanent link}

http://nrs.harvard.edu/urn-3:HUL.InstRepos:30212166

\section{Terms of Use}

This article was downloaded from Harvard University's DASH repository, and is made available under the terms and conditions applicable to Other Posted Material, as set forth at http:// nrs.harvard.edu/urn-3:HUL.InstRepos:dash.current.terms-of-use\#LAA

\section{Share Your Story}

The Harvard community has made this article openly available.

Please share how this access benefits you. Submit a story.

Accessibility 
THE ASTROPHYSICAL JouRNAL, 513:76-107, 1999 March 1

(C) 1999. The American Astronomical Society. All rights reserved. Printed in U.S.A.

\title{
INVESTIGATION OF THE RELATION BETWEEN THE SPECTRAL ENERGY DISTRIBUTIONS AND EMISSION LINES IN LOW-REDSHIFT QUASARS ${ }^{1}$
}

\author{
Belinda J. Wilkes, Joanna Kuraszkiewicz, ${ }^{2}$ Paul J. Green, Smita Mathur, and Jonathan C. McDowell \\ Harvard-Smithsonian Center for Astrophysics, Cambridge, MA 02138 \\ Received 1997 December 23; accepted 1998 October 6
}

\begin{abstract}
We investigate the relations between the observed emission-line strengths, widths, and continuum properties of a sample of 41 low-redshift $(z<1)$ quasars for which contemporaneous IR/soft X-ray spectral energy distributions are available. This includes investigating correlations between optical and UV lines with both the luminosity and the shape of the quasars' continuum, as well as correlations between the various lines. The sample is heterogeneous, primarily selected on the existence of good-quality Einstein X-ray data, and includes 18 radio-loud and 23 radio-quiet quasars. We find anticorrelations between the equivalent width and various UV luminosities (the Baldwin effect) for the Ly $\alpha$ and $\mathrm{H} \beta$ lines and a marginal anticorrelation for $\mathrm{C}$ III]. Exclusion of narrow-line, low-luminosity active galactic nuclei reveals a significant Baldwin effect for the $\mathrm{C}$ IV and $\mathrm{C}$ III] lines. A significant anticorrelation of $\mathrm{EW}(\mathrm{C}$ IV) with $\alpha_{o x}$ is also present. We find no correlations between any lines and the X-ray luminosity or X-ray slope. The $\mathrm{Fe}$ II optical multiplet shows no simple relationship with luminosity or continuum slope; however, there is a tendency for objects with flat X-ray spectra and/or strong X-ray luminosities to have weak Fe II.

Our data do not favor a model in which changes in continuum shape (due to, e.g., a decreasing ionization parameter) cause the Baldwin effect. The data can instead be explained by an accretion disk (AD) model in which limb darkening and the projected surface area of an optically thick, geometrically thin disk combine to cause a viewing-angle-dependent UV luminosity and a more isotropic X-ray luminosity. The scatter in our correlations is larger than that expected from this AD model, suggesting the presence of dust, which reddens both the continuum and the broad emission lines. The C IV and C III] lines show flatter slopes and larger scatter in the line-continuum relations than predicted by the $\mathrm{AD}+$ dusty torus model. This may be due to a significant contribution from collisional excitation that is not directly related to the ionizing continuum.
\end{abstract}

Subject headings: galaxies: active - quasars: emission lines - quasars: general

\section{INTRODUCTION}

The strong, broad emission lines that characterize quasar spectra are generally believed to be generated in a large number of small gas clouds photoionized by the central continuum source of a quasar. This region is known as the broad emission line region (BELR). To date, photoionization models have been reasonably successful in predicting the average emission-line properties of a quasar using an average continuum shape. However, it has become clear that, while the emission-line properties are largely similar from quasar to quasar, the observed spectral energy distributions (SEDs) are not (Elvis et al. 1994, hereafter E94). If photoionization models are generally applicable, we would expect systematic relations between the observed lines and continuum in different objects (Krolik \& Kallman 1988) and, at first glance at least, the dichotomy between continuum and the line behavior looks surprising.

The most enduring relation between the lines and continua in quasars is the inverse correlation between line equivalent width (EW) and continuum luminosity, known as the Baldwin effect (Baldwin 1977). This relation is not predicted in a simple photoionization scenario, and explanations range from luminosity-dependent continuum shape, which

\footnotetext{
${ }^{1}$ Observations reported here were obtained at the Multiple Mirror Telescope Observatory, a facility operated jointly by the University of Arizona and the Smithsonian Institution.

${ }^{2}$ Also N. Copernicus Astronomical Center, ul. Bartycka 18, 00-716 Warsaw, Poland.
}

affects the BELR ionization, to geometric effects. Another intriguing set of correlations, which have caused a flurry of activity in recent years, centers around an inverse relation between the strengths of the broad $\mathrm{Fe}$ II $\lambda 4570$ and narrow [O III] $\lambda 5007$ lines and includes soft X-ray slope and luminosity and broad line width (Boroson \& Green 1992; Boller, Brandt, \& Fink 1996; Lawrence et al. 1997). Again, a simple photoionization scenario fails. The current popular explanation involves the mass flow into the central black hole; this is attractive, as it would imply an observational link into the central power house (Pounds, Done, \& Osbourne 1996). Despite the general success of photoionization models, these results suggest that other factors also play a role in determining the emission-line properties of quasars.

Most studies of line and continuum properties of quasars to date have centered around large samples, often including nonuniform data sets from a variety of sources and generally utilizing global continuum parameters such as $L_{\mathrm{opt}}, L_{\mathrm{x}}$ and $\alpha_{o x}$. Since the photoionization of any individual ion and its resulting relation with the ionizing continuum is unique to that ion, the use of global parameters has obvious limitations for investigating photoionization models. The current study takes a different, complementary approach in concentrating on uniform, high-quality continuum and emission-line data for a small sample of quasars. This data set facilitates a study of the relation of each emission line to various parts of the continuum and, potentially, allows differentiation between ionization and other effects. 
We have obtained far-IR through soft X-ray $(100 \mu \mathrm{m}-3.5$ $\mathrm{keV}$ ) continuum data for a sample of 41 quasars. SEDs for 30 of these objects were presented in E94; the remaining 11 are compiled here from a combination of our own and published data. We also present low-resolution $(5-20 \AA)$ optical spectrophotometry that was obtained within a few months of the optical/IR continuum measurements. UV spectra from IUE and/or the Hubble Space Telescope (HST) have been obtained either by ourselves or from their respective archives. The range in shapes of the IR-UV continuum is large even in this small, low-redshift sample (E94), allowing investigation of whether the range in continuum shapes produces any corresponding range in the emissionline properties.

\section{OBSERVATIONS AND DATA REDUCTION}

\subsection{The Sample}

Our sample is a heterogeneous sample of 41 low-redshift quasars $(z<1)$, where 23 are radio quiet (RQQ) and 18 are radio loud ( $R L Q)$. The radio-quiet objects dominate at lower redshifts, while the radio-loud dominate at higher redshifts (see Fig. 1). All but three quasars (PG 1012+008, PG $1121+422$, and PG 2304+042) have Einstein X-ray observations with sufficient counts to define the X-ray spectral index. This selection introduces a bias toward objects with strong X-ray emission relative to the optical (small $\alpha_{o x}$ ). The SEDs for 30 objects were presented in E94. For the remaining 11 objects the SEDs were assembled from existing data as described below. The list of all quasars with their redshifts is given in Table 1 .

\subsection{Optical Spectroscopy}

The quasars were observed during the period 1985-1991 mostly with the Multiple Mirror Telescope (MMT) on Mount Hopkins in Arizona. Both blue and red spectrographs were used as far as possible to provide full coverage (3200-8400 ̊) of the optical continuum. Southern objects

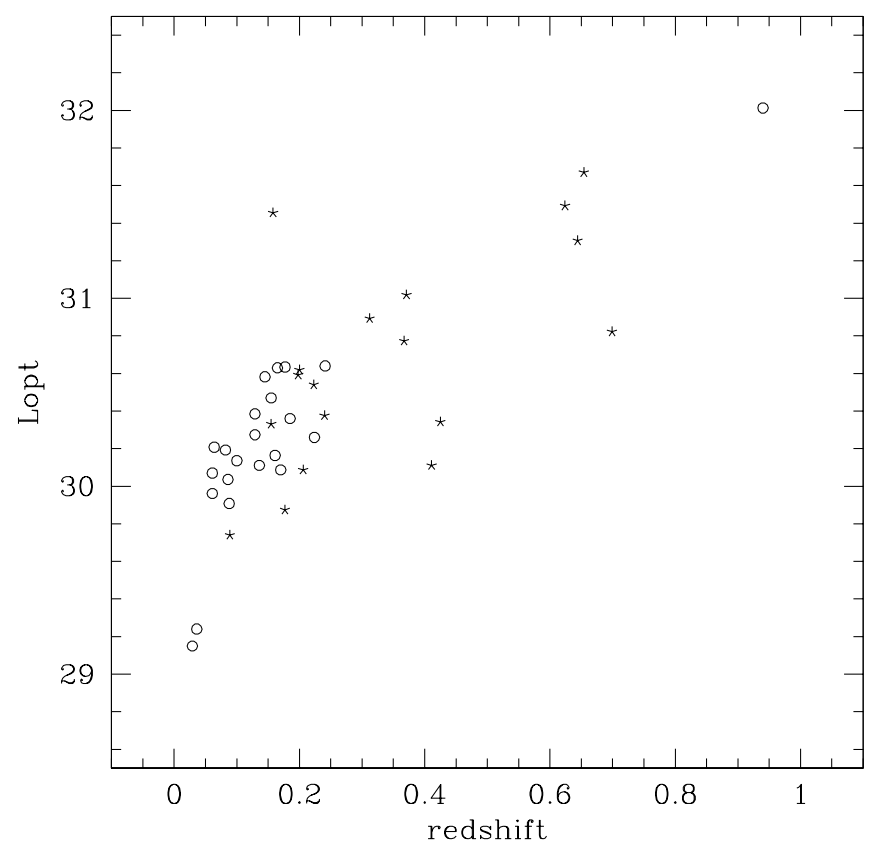

FIG. 1. $-L_{\text {opt }}(2500 \AA$ luminosity) vs. redshift for our sample. In this and all subsequent figures, stars indicate RLQs and circles RQQs. were observed with the Cerro Tololo Inter-American Observatory (CTIO) $1.5 \mathrm{~m}$ telescope and CCD spectrograph. The observational details are given in Table 1, including the instrument and wavelength range covered in each case.

In photometric conditions, spectrophotometry was carried out by observing each quasar twice, first through a large aperture, $\gtrsim 5^{\prime \prime}$, and second through a small aperture, $\sim 1 " 5$, for a longer time in order to obtain high spectral resolution and signal-to-noise ratio $(\mathrm{S} / \mathrm{N})$. A standard star was observed through the wide aperture, at similar air mass, immediately before or after the quasar observation to provide flux calibration. The data were reduced in the standard manner using IRAF. The continuum of the small aperture data was then normalized to match the shape and absolute flux level of the large aperture observation, yielding a final spectrum with resolution $\sim 5-20 \AA$ (depending on the instrument) and a photometric accuracy of $\sim 5 \%$. Except where noted in Table 1, all spectra have been flux calibrated using a large-aperture spectrum and observed in photometric conditions as described above. The date of the spectrophotometry, sometimes different from that of the higher $\mathrm{S} / \mathrm{N}$, small-aperture spectrum, is also noted in Table 1.

In some cases several spectra covering largely overlapping wavelength ranges were obtained. If no significant change in continuum shape was apparent, these spectra were combined to improve the final S/N. Spectra with continuum shapes that did not agree within the errors were not combined, instead the discrepant or lower quality spectrum was discarded. Blue and red spectra of the same object were not combined since this adds uncertainty in the small overlap region of the spectra and is not required for our analysis. Table 1 lists observational details for all the individual spectra (i.e., before being combined). The final, combined spectra are presented in Figure 2 in order of increasing right ascension with blue and red spectra displayed separately.

The fluxes, EW, and FWHM were measured for all the prominent optical and UV emission lines: $\mathrm{O}$ VI $\lambda 1034+\operatorname{Ly} \beta \lambda 1025, \quad \operatorname{Ly} \alpha \lambda 1216+\mathrm{N} \quad \mathrm{v} \lambda 1240, \quad \mathrm{Si}$ IV $\lambda 1397+$ O IV] $\lambda 1402, \mathrm{C}$ IV $\lambda 1549, \mathrm{C}$ III] $\lambda 1909+\mathrm{Si}$ III] $\lambda 1892+\mathrm{Al}$ III $\lambda 1857, \mathrm{Mg}$ II $\lambda 2798,[\mathrm{Ne}$ v] $\lambda 3426,[\mathrm{O}$ II] $\lambda 3727,\left[\mathrm{Ne}\right.$ III] $\lambda 3869+\mathrm{He}_{\mathrm{I}} \lambda 3889,[\mathrm{Ne}$ III] $\lambda 3967, \mathrm{H} \delta$ $\lambda 4102, \mathrm{H} \gamma \lambda 4340, \mathrm{Fe}$ II $\lambda 4570, \mathrm{H} \beta \lambda 4861$, and [O III] $\lambda 5007$. The EW and fluxes were measured by the summation procedure detailed in Robertson (1986), which provides a better estimate of the line wings than Gaussian fitting. All fluxes and EW were remeasured using the SPLOT task in IRAF. Rather than fitting a Gaussian function to these lines, which frequently does not work well, we measured the actual data by fitting a linear continuum and integrating across the observed emission line. If the EW and fluxes derived by both methods agreed within $10 \%$, the values obtained by the summation procedure were used for the subsequent analysis. If they disagreed, the values from the SPLOT task were used. For the Fe II optical multiplet, which is especially difficult to measure if the line is weak, the summation procedure was used to determine the EW. The FWHMs were measured using only the IRAF SPLOT task by fitting a linear continuum, measuring the line peak flux above the continuum, determining the half-maximum intensity, and measuring the width at that level. By measuring all the data ourselves, we minimize the scatter generally introduced by 
TABLE 1

OBSERVATIONAL DETAILS

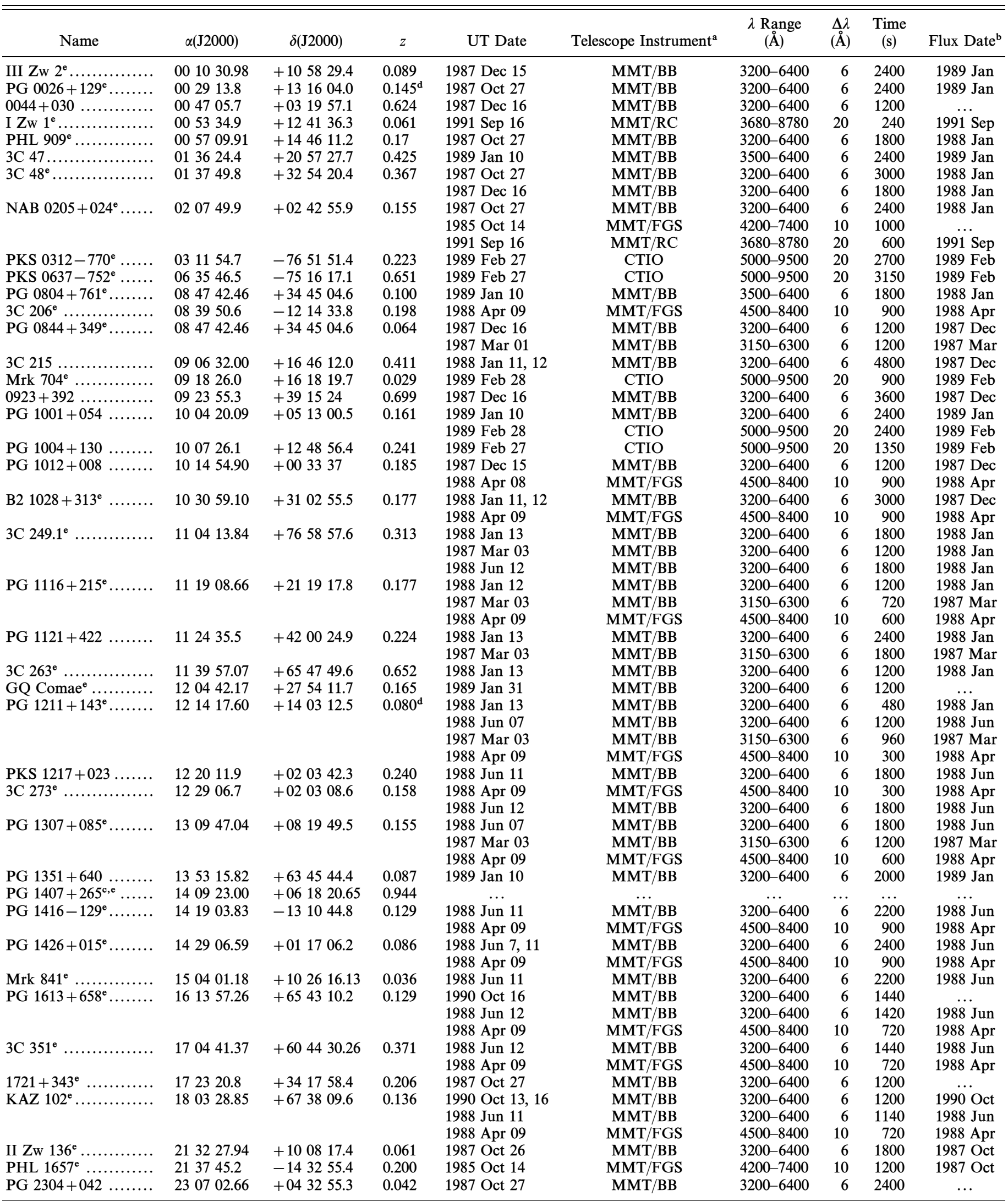

NoTE- - Units of right ascension are hours, minutes, and seconds, and units of declination are degrees, arcminutes, and arcseconds.

a MMT: Multiple Mirror Telescope; FGS: Faint Object Grism CCD Spectrograph; BB: Big Blue Reticon; RC: Red channel, CCD spectrograph; CTIO: $1.5 \mathrm{~m}$ plus CCD spectrograph.

${ }^{b}$ Date (month) of spectrophotometry used to flux calibrate this spectrum.

c Data already published, McDowell et al. (1995).

${ }^{d}$ Redshift revised based on our spectra.

e Radio-X-ray SED in Elvis et al. (1994). 
combining data sets from different measuring techniques and different authors. The EWs of optical and UV lines are listed in Tables 2 and 3, the UV and optical line fluxes in Tables 4 and 5, and the UV and optical FWHM in Tables 6 and 7 . All values are in the rest frame.

\subsection{Spectral Energy Distributions}

The SEDs for the 11 quasars not presented in E94 were compiled here from a combination of our own groundbased optical and near-IR photometry (from the $0.6 \mathrm{~m}$ Fred Lawrence Whipple Observatory telescope, the MMT, the Mount Lemmon 61" telescope, and the IRTF; for details see Table 8 and E94), as well as from the Einstein X-ray data, archival IUE or HST UV data, and IRAS far-IR data. The various data sets were combined following the method described in E94. When no optical photometry was available the MMT spectrophotometry was used. For inclusion in the SEDs, prominent emission lines were subtracted from the optical and UV spectrum and the spectrum binned into broader wavelength bands. A correction for Galactic extinction was applied to the SEDs based on the Galactic neutral hydrogen column and assuming a fixed conversion of $N(\mathrm{H} \mathrm{I}) / E(B-V)=5.0 \times 10^{21} \mathrm{~cm}^{2} \mathrm{mag}^{-1}$ (Burstein \& Heiles 1978). The Galactic H I column has been accurately measured by Elvis et al. (1986) for seven of the 11 objects. For the remaining four objects it was determined using the dereddening values from Neugebauer et al. (1987). After correcting for Galactic extinction, the data were blueshifted to the rest frame using a cosmological model with $\Omega_{0}=1$ and $H_{0}=50 \mathrm{~km} \mathrm{~s}^{-1} \mathrm{Mpc}^{-1}$. No $k$-corrections and no assumptions about the intrinsic spectrum were required, since we were working with the complete SEDs. The contribution from the host galaxy was then subtracted using the method and template of E94 (based on the Sbc galaxy model of Coleman, Wu, \& Weedman 1980) and normalized by the host galaxy monochromatic luminosity in the $H$ band, which was taken from the literature or, when not available, set to $\frac{1}{3}$ of the luminosity of the quasar in that band (McLeod \& Rieke 1994a, 1994b). The final SEDs for the 11 objects are displayed in Figure 3 in order of right ascension.

To allow comparison with earlier studies, in Table 9 we list several standard continuum parameters taken from the literature and determined in the usual way (i.e., not from the SEDs). These include: $L_{\text {opt }}$ (luminosity at $2500 \AA$ ),$L_{\mathrm{x}}$ (X-ray luminosity at $2 \mathrm{keV}$ ), $\alpha_{\mathrm{ox}}$ (effective optical-to-X-ray slope) from Wilkes et al. (1994), $\alpha_{\mathrm{x}}$ (X-ray energy index, where $f_{v} \propto v^{-\alpha_{\mathrm{x}}}$ ) from Wilkes \& Elvis (1987), $\alpha_{\text {ouv }}$ (optical slope, $f_{v} \propto v^{-\alpha_{\text {ouv }}}$ taken between 1285 and $5100 \AA$ ) from Kuhn (1996), and radio class (quasar considered radio-loud [L] if $R_{L}>1$, after E94). We also list the blue-bump strength measured from the SEDs, $C_{\mathrm{UV} / \mathrm{R}}$ (E94). In order to better characterize the SEDs, we directly measured the decade and octave luminosities, as defined in E94 (given in Tables 10
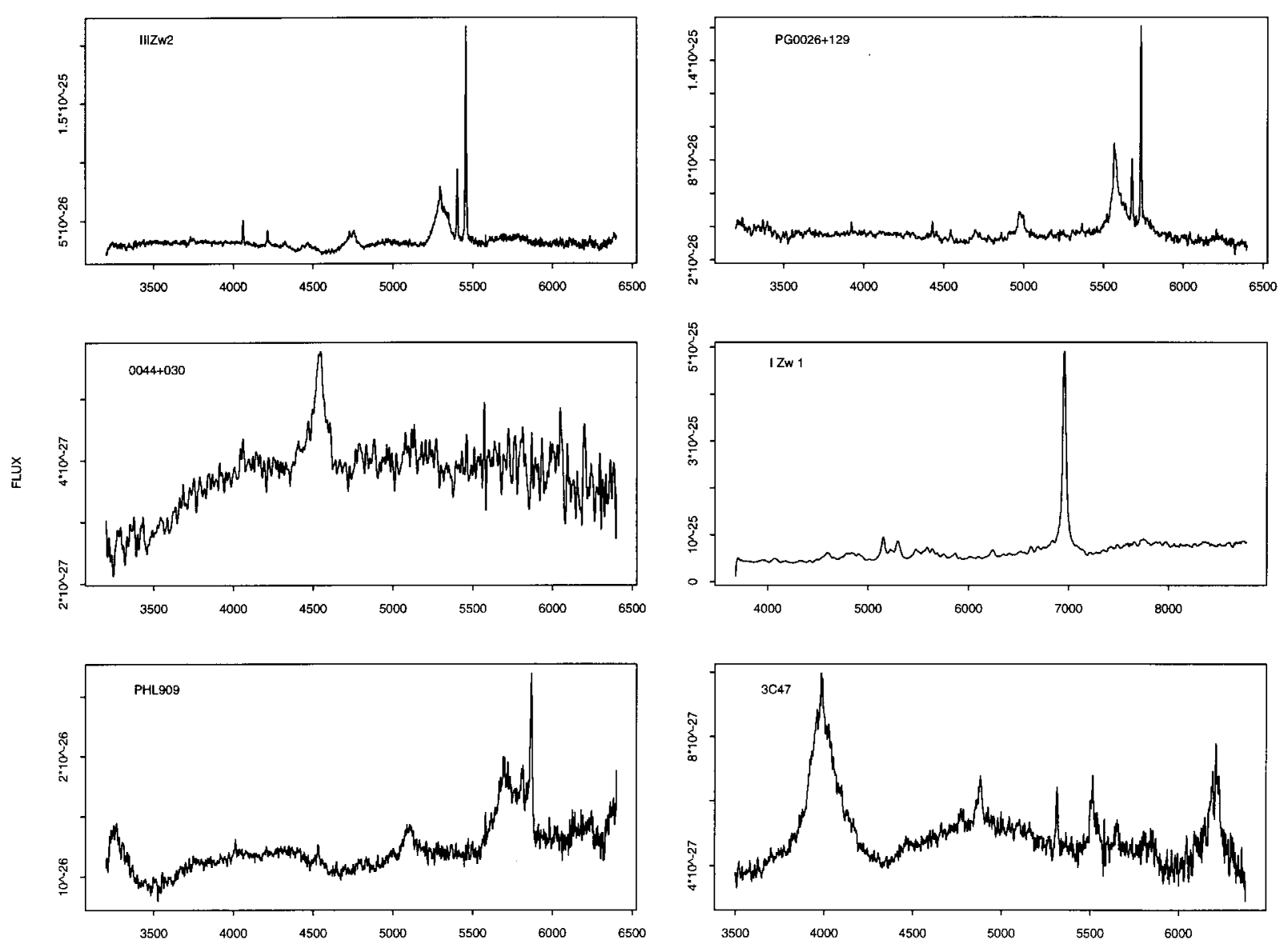

WAVELENGTH

FIG. 2. - Optical spectrophotometry of the quasars in our sample in order of increasing right ascension and on a scale of $F_{v}\left(\mathrm{ergs} \mathrm{cm}^{-2} \mathrm{~s}^{-1} \mathrm{~Hz}^{-1}\right)$ as a function of $\lambda(\AA)$. Observational details are given in Table 1. 

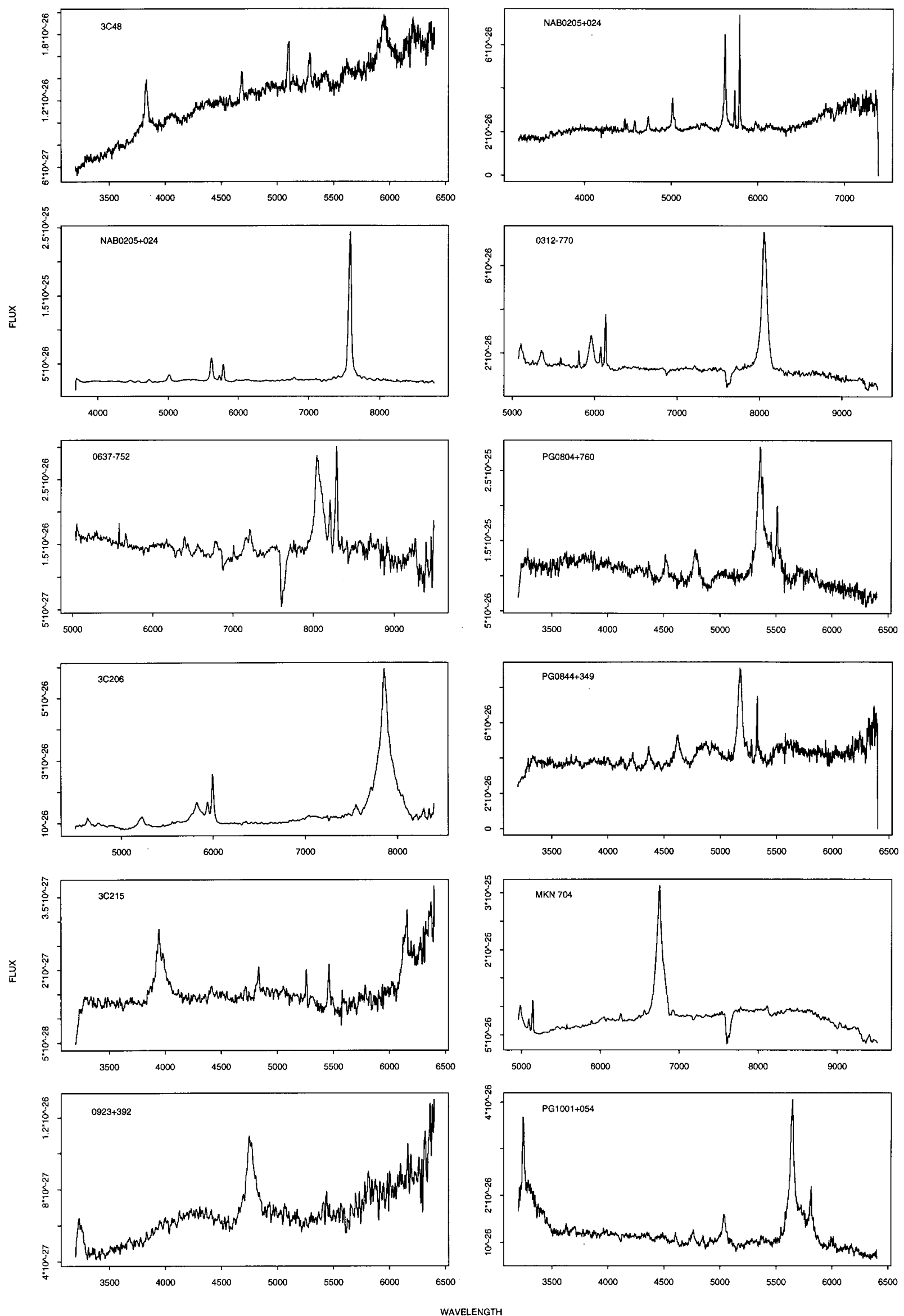

Fig. 2.-Continued 

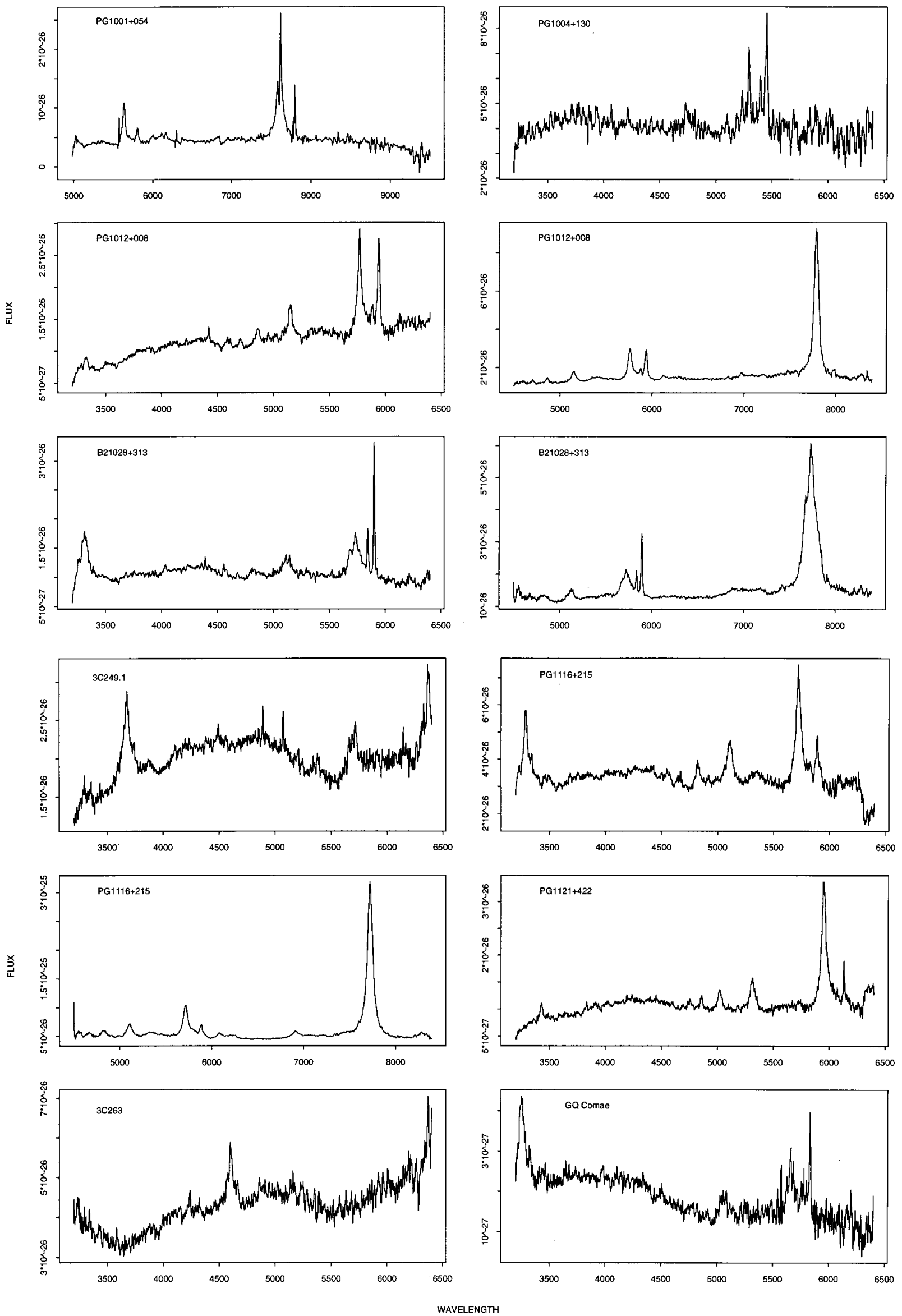

FIG. 2.-Continued 

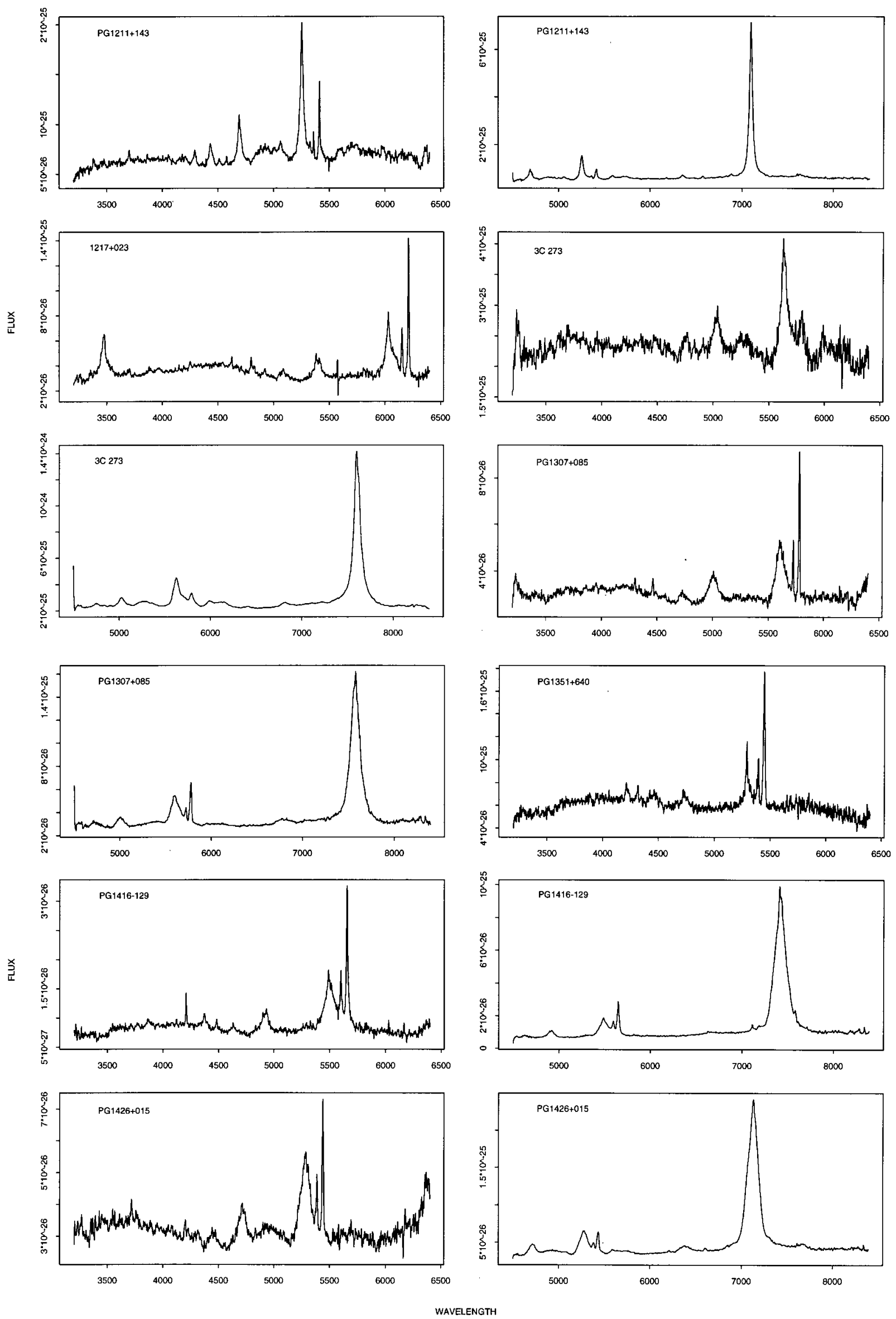

FIG. 2.-Continued 

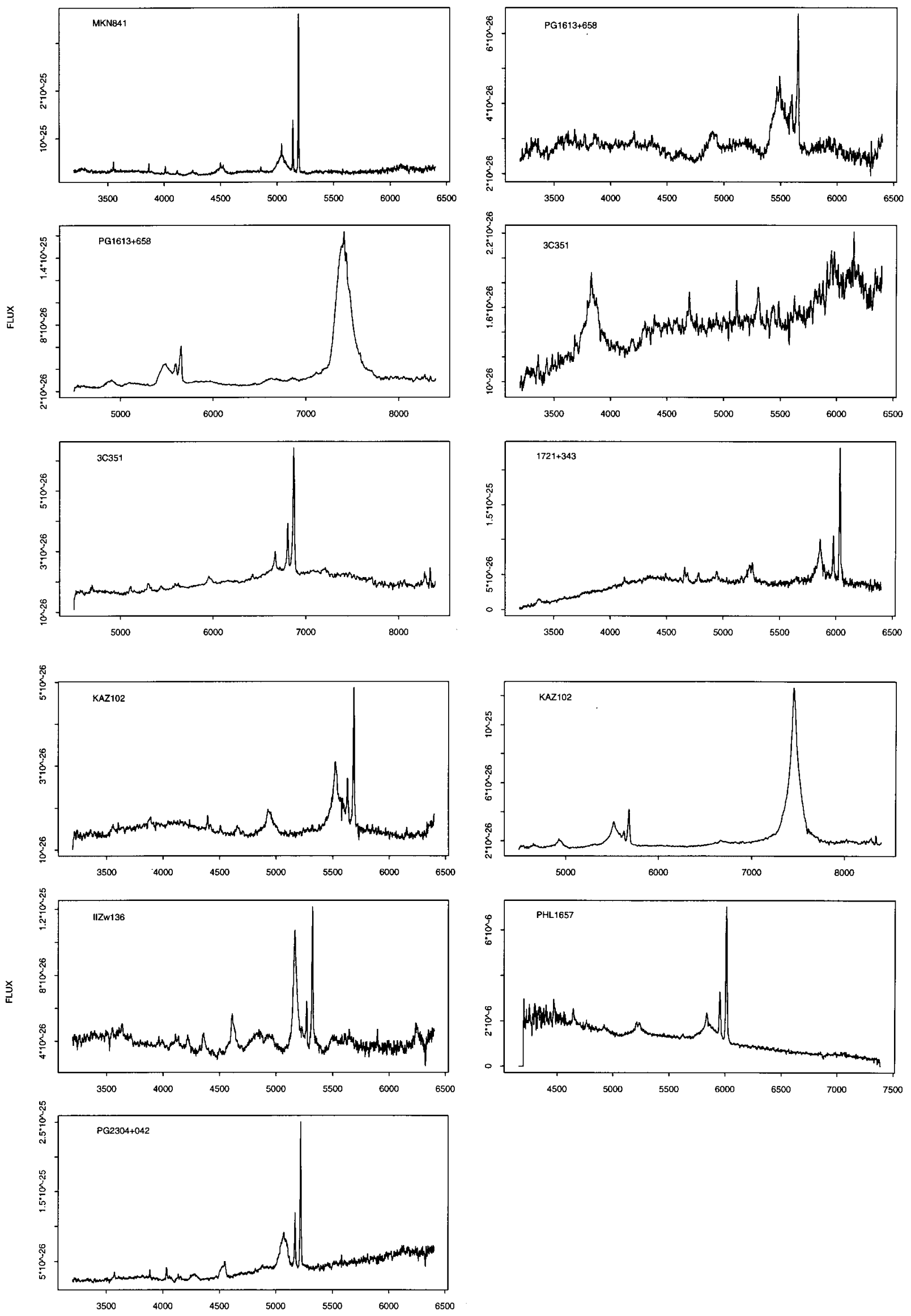

WAVELENGTH

Fig. 2.-Continued 
TABLE 2

UV-Line Rest Frame Equivalent Widths in A

\begin{tabular}{|c|c|c|c|c|c|}
\hline Name & Ly $\alpha$ N v $\lambda 1216$ & $\operatorname{Ly} \beta$ O vi $\lambda 1034$ & C IV $\lambda 1549$ & Si IV O IV] $\lambda 1400$ & $\mathrm{Al}$ III C III] $\lambda 1909$ \\
\hline $0007+106 \ldots \ldots$ & 84.300 & $\ldots$ & 116.100 & 23.900 & 31.900 \\
\hline $0026+129 \ldots \ldots$ & 64.300 & $\ldots$ & 34.700 & $<8.400$ & $<29.000$ \\
\hline $0044+030 \ldots \ldots$ & 63.400 & $<15.000$ & 50.800 & 50.300 & 27.900 \\
\hline $0050+124 \ldots \ldots$ & 171.400 & $\ldots$ & 39.900 & 39.700 & 36.000 \\
\hline $0054+145 \ldots \ldots$ & 87.400 & $\ldots$ & 112.600 & $<30.700$ & $<79.000$ \\
\hline $0133+207 \ldots \ldots$ & $\ldots$ & $\ldots$ & $\ldots$ & $\ldots$ & $\ldots$ \\
\hline $0134+329 \ldots \ldots$ & $<24.700$ & $<14.700$ & $<111.000$ & $<16.800$ & $<20.500$ \\
\hline $0205+024 \ldots \ldots$ & 60.900 & $\ldots$ & 38.500 & $<9.000$ & $<38.700$ \\
\hline $0312-770 \ldots \ldots$ & 72.600 & 24.700 & $<83.100$ & $<13.800$ & $<285.200$ \\
\hline $0637-752 \ldots \ldots$ & 123.800 & 7.100 & 61.500 & $<121.500$ & $<13.900$ \\
\hline $0804+761 \ldots \ldots$ & 101.800 & $\ldots$ & 81.700 & 28.000 & 63.700 \\
\hline $0837-120 \ldots \ldots$ & 87.600 & $\ldots$ & 91.400 & $<16.500$ & $<41.400$ \\
\hline $0844+349 \ldots \ldots$ & 113.600 & $\ldots$ & 18.500 & 27.900 & $<15.300$ \\
\hline $0903+169 \ldots \ldots$ & $\ldots$ & $\ldots$ & $\ldots$ & $\ldots$ & $\ldots$ \\
\hline $0915+165 \ldots \ldots$ & $\ldots$ & $\ldots$ & $\ldots$ & $\ldots$ & $\ldots$ \\
\hline $0923+392 \ldots \ldots$ & $\ldots$ & $\ldots$ & $\ldots$ & $\ldots$ & $\ldots$ \\
\hline $1001+054 \ldots \ldots$ & $\ldots$ & $\ldots$ & $\ldots$ & $\ldots$ & $\ldots$ \\
\hline $1004+130 \ldots \ldots$ & 9.100 & $<10.600$ & $<66.500$ & $<12.600$ & $<77.700$ \\
\hline $1012+008 \ldots \ldots$ & 72.900 & $\ldots$ & 13.300 & $<13.500$ & $\ldots$ \\
\hline $1028+313 \ldots \ldots$ & 79.200 & $\ldots$ & 70.800 & $<12.100$ & $<31.600$ \\
\hline $1100+772 \ldots \ldots$ & 57.800 & 7.700 & 90.800 & $<4.500$ & $<19.800$ \\
\hline $1116+215 \ldots \ldots$ & 62.900 & $\ldots$ & 36.500 & $<4.100$ & 31.200 \\
\hline $1121+422 \ldots \ldots$ & $\ldots$ & $\ldots$ & $\ldots$ & $\ldots$ & $\ldots$ \\
\hline $1137+661 \ldots \ldots$ & 75.300 & 13.100 & 44.800 & 46.700 & $<46.600$ \\
\hline $1202+281 \ldots \ldots$ & 74.200 & $\ldots$ & 129.100 & $<3.600$ & $<46.600$ \\
\hline $1211+143 \ldots \ldots$ & 100.800 & $\ldots$ & 38.800 & 14.600 & 17.000 \\
\hline $1217+023 \ldots \ldots$ & 138.200 & 28.300 & 173.000 & 38.900 & $<35.500$ \\
\hline $1226+023 \ldots \ldots$ & 46.100 & $\ldots$ & 36.600 & 4.800 & 10.000 \\
\hline $1307+085 \ldots \ldots$ & 76.100 & $\ldots$ & 93.700 & $<15.100$ & $<40.400$ \\
\hline $1351+640 \ldots \ldots$ & 70.900 & $\ldots$ & 30.300 & 25.500 & 25.400 \\
\hline $1407+265 \ldots \ldots$ & 8.000 & $\ldots$ & 4.100 & $\ldots$ & 9.900 \\
\hline $1416-129 \ldots \ldots$ & 134.100 & $\ldots$ & 181.800 & $<18.100$ & $<292.700$ \\
\hline $1426+015 \ldots \ldots$ & 44.100 & $\ldots$ & 37.100 & 12.000 & 16.200 \\
\hline $1501+106 \ldots \ldots$ & $\ldots$ & $\ldots$ & $\ldots$ & $\ldots$ & $\ldots$ \\
\hline $1613+658 \ldots \ldots$ & 89.300 & $\ldots$ & 104.900 & $<6.600$ & 33.300 \\
\hline $1704+608 \ldots \ldots$ & 33.600 & $<3.600$ & 49.800 & $<2.300$ & 13.800 \\
\hline $1721+343 \ldots \ldots$ & 126.300 & 30.200 & 94.000 & $<5.000$ & $<23.900$ \\
\hline $1803+676 \ldots \ldots$ & 95.800 & $\ldots$ & 133.300 & 9.600 & 34.400 \\
\hline $2130+099 \ldots \ldots$ & 110.400 & $\ldots$ & 56.600 & 23.300 & 18.200 \\
\hline $2135-147 \ldots \ldots$ & 88.300 & $\ldots$ & 49.500 & $<6.800$ & $<50.300$ \\
\hline $2304+042 \ldots \ldots$ & $\ldots$ & $\ldots$ & $\ldots$ & $\ldots$ & $\ldots$ \\
\hline
\end{tabular}

and 11). In Table 12 we give several broadband luminosities: $L_{\mathrm{UVOIR}}, L_{\mathrm{bol}}, L_{\mathrm{Lycon}}$, and $L_{\mathrm{Ion}}$ (ionizing continuum), all defined as in E94. All SED parameters were measured using the TIGER software package written by J. McDowell (Wilkes \& McDowell 1995). We have also measured the driving continuum for each line following Krolik \& Kallman (1988; Table 2).

\section{CORRELATION ANALYSIS}

We studied correlations between the various line and continuum properties in Tables 13 and 14 using the ASURV statistical package (Isobe, Feigelson, \& Nelson 1986), which includes allowance for the presence of upper limits in the sample. Specifically, we applied the following tests to each pair of parameters: the generalized Kendall rank test and the Spearman rank test, which is insensitive to outlying points. We considered a correlation real only if the probability of it occurring by chance was less than $2 \%$ in both of these tests. We present the percentage probability of a chance correlation in the generalized Kendall rank test followed by that for the Spearman rank test in Tables 13 and 14 for probabilities less than 5\%. Significant correlations $(P<2 \%)$ are in boldface type. When multiple correlations were found, we tested for the primary relation using ASURV bivariate Spearman ranks as input to partial Spearman rank analysis (Kendal \& Stuart 1976). The primary correlations, i.e., the strongest pairwise correlations when the other variables are held constant, are italicized in Tables 13 and 14.

Since we are considering a large number of parameters (three parameters for each of 15 emission lines: EW, FWHM, flux, and 33 continuum parameters; see previous section), we divide our results up by emission-line parameters, first discussing the $\mathrm{EW}$ versus continuum luminosity correlations (§3.1), followed by the flux (§ 3.2) and the FWHM (§ 3.3). We then discuss the correlations between the various line parameters $(\S 3.4)$ and between the various continuum parameters $(\S 3.5)$. The number of correlations studied and found and the number of spurious correlations expected with $P<2 \%$ are shown in Table 15 . 


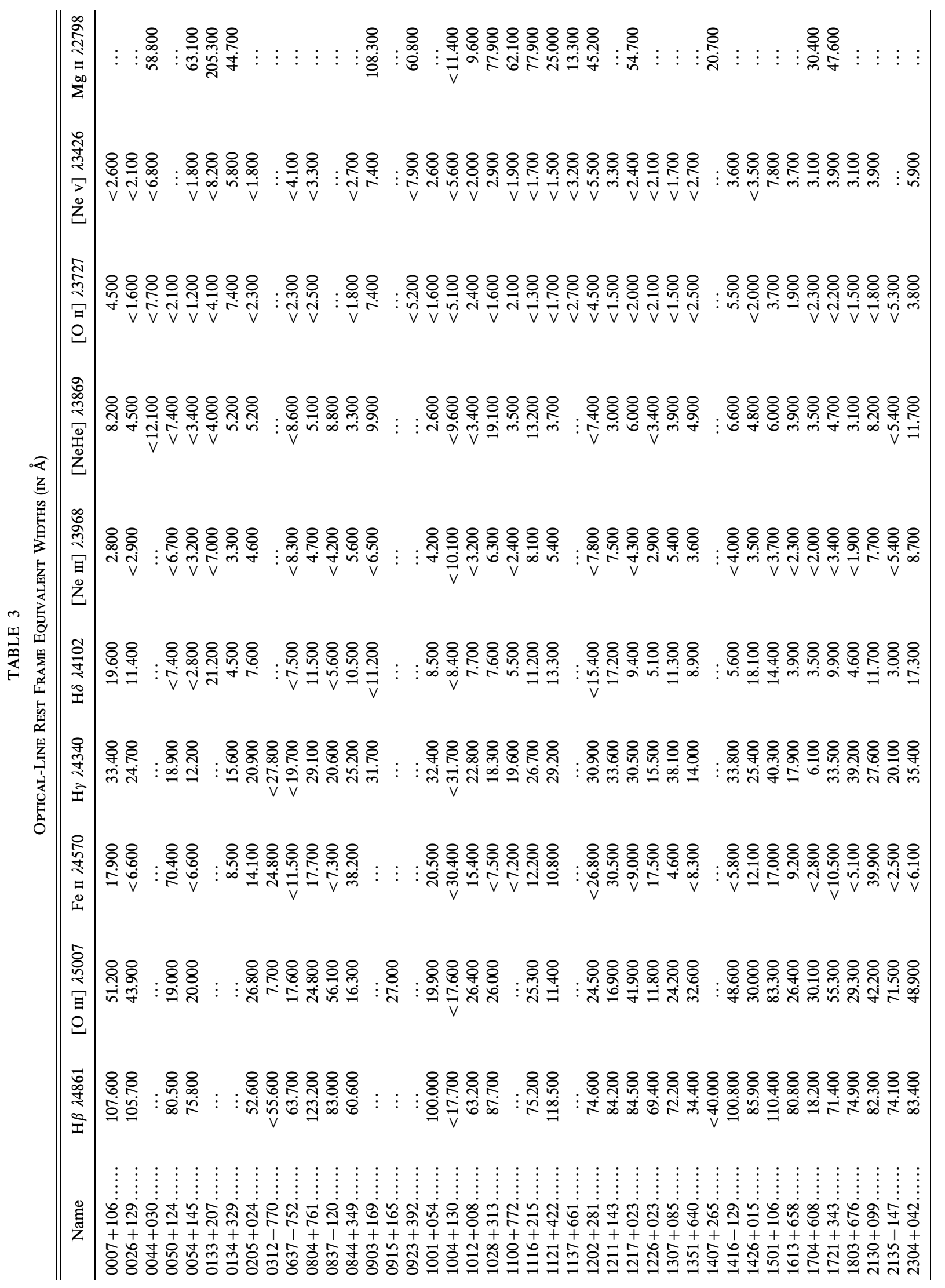


TABLE 4

UV LINE FLUXES ${ }^{\mathrm{a}}$

\begin{tabular}{|c|c|c|c|c|c|}
\hline Name & $\log F(\operatorname{Ly} \alpha \mathrm{N} v)$ & $\log F(\operatorname{Ly} \beta \mathrm{O} \mathrm{vI})$ & $\log F(\mathrm{C}$ IV $)$ & $\log F($ Si IV O IV] $)$ & $\log F(\mathrm{Al}$ III $\mathrm{C}$ III] $])$ \\
\hline $0007+106 \ldots \ldots$ & -11.583 & $\ldots$ & -11.652 & -12.296 & -12.362 \\
\hline $0026+129 \ldots \ldots$ & -11.619 & $\ldots$ & -12.069 & $<-12.613$ & $<-12.166$ \\
\hline $0044+030 \ldots \ldots$ & -11.499 & $<-12.224$ & -11.695 & -11.749 & -12.074 \\
\hline $0050+124 \ldots \ldots$ & -11.369 & $\ldots$ & -12.077 & -12.123 & -12.144 \\
\hline $0054+145 \ldots \ldots$ & -11.989 & $\ldots$ & -12.124 & $<-12.636$ & $<-12.332$ \\
\hline $0133+207 \ldots \ldots$ & $\ldots$ & $\ldots$ & $\ldots$ & $\ldots$ & $\ldots$ \\
\hline $0134+329 \ldots \ldots$ & $<-12.434$ & $<-12.787$ & $<-12.132$ & $<-12.842$ & $<-12.889$ \\
\hline $0205+024 \ldots \ldots$ & -11.780 & $\ldots$ & -12.168 & $<-12.732$ & $<-12.099$ \\
\hline $0312-770 \ldots \ldots$ & -11.650 & -12.088 & $<-11.825$ & $<-12.469$ & $<-11.209$ \\
\hline $0637-752 \ldots \ldots$ & -11.167 & -12.318 & -11.564 & $<-11.455$ & $<-12.158$ \\
\hline $0804+761 \ldots \ldots$ & -11.055 & $\ldots$ & -11.378 & -11.793 & -11.477 \\
\hline $0837-120 \ldots \ldots$ & -11.748 & $\ldots$ & -11.867 & $<-12.603$ & $<-12.231$ \\
\hline $0844+349 \ldots \ldots$ & -11.521 & $\cdots$ & -12.364 & -12.192 & $<-12.565$ \\
\hline $0903+169 \ldots \ldots$ & $\ldots$ & $\ldots$ & $\ldots$ & $\ldots$ & $\ldots$ \\
\hline $0915+165 \ldots \ldots$ & $\ldots$ & $\ldots$ & $\ldots$ & $\ldots$ & $\ldots$ \\
\hline $0923+392 \ldots \ldots$ & $\ldots$ & $\ldots$ & $\ldots$ & $\ldots$ & $\ldots$ \\
\hline $1001+054 \ldots \ldots$ & $\ldots$ & $\ldots$ & $\ldots$ & $\ldots$ & $\ldots$ \\
\hline $1004+130 \ldots \ldots$ & -12.690 & $<-12.808$ & $<-12.102$ & $<-12.705$ & $<-11.814$ \\
\hline $1012+008 \ldots \ldots$ & -12.082 & $\ldots$ & -12.916 & $<-12.896$ & - \\
\hline $1028+313 \ldots \ldots$ & -11.807 & $\ldots$ & -12.014 & $<-12.727$ & $<-12.248$ \\
\hline $1100+772 \ldots \ldots$ & -11.647 & -12.520 & -11.691 & $<-12.919$ & $<-12.418$ \\
\hline $1116+215 \ldots \ldots$ & -11.201 & $\ldots$ & -11.593 & $<-12.501$ & -11.734 \\
\hline $1121+422 \ldots \ldots$ & $\ldots$ & $\ldots$ & $\ldots$ & $\ldots$ & $\ldots$ \\
\hline $1137+661 \ldots \ldots$ & -11.533 & -12.279 & -11.910 & -11.867 & $<-11.871$ \\
\hline $1202+281 \ldots \ldots$ & -11.949 & $\ldots$ & -11.934 & $<-13.385$ & $<-12.313$ \\
\hline $1211+143 \ldots \ldots$ & -11.256 & $\ldots$ & -11.778 & -12.170 & -12.265 \\
\hline $1217+023 \ldots \ldots$ & -11.741 & -12.470 & -11.910 & -12.506 & $<-12.663$ \\
\hline $1226+023 \ldots \ldots$ & -10.807 & $\ldots$ & -11.103 & -11.921 & -11.789 \\
\hline $1307+085 \ldots \ldots$ & -11.519 & $\ldots$ & -11.696 & $<-12.404$ & $<-12.100$ \\
\hline $1351+640 \ldots \ldots$ & -11.677 & $\ldots$ & -12.196 & -12.269 & -12.318 \\
\hline $1407+265 \ldots \ldots$ & $\ldots$ & $\ldots$ & $\ldots$ & $\ldots$ & \\
\hline $1416-129 \ldots \ldots$ & -11.648 & $\ldots$ & -11.683 & $<-12.619$ & $<-11.958$ \\
\hline $1426+015 \ldots \ldots$ & -11.249 & $\ldots$ & -11.573 & -12.013 & -12.108 \\
\hline $1501+106 \ldots \ldots$ & $\ldots$ & $\ldots$ & $\ldots$ & $\ldots$ & $\ldots$ \\
\hline $1613+658 \ldots \ldots$ & -11.653 & $\ldots$ & -11.789 & $<-12.896$ & -12.344 \\
\hline $1704+608 \ldots \ldots$ & -12.019 & $<-13.018$ & -12.085 & $<-13.360$ & -12.715 \\
\hline $1721+343 \ldots \ldots$ & -11.265 & -11.888 & -11.518 & $<-12.764$ & $<-12.314$ \\
\hline $1803+676 \ldots \ldots$ & -11.483 & $\ldots$ & -11.603 & -12.679 & -12.258 \\
\hline $2130+099 \ldots \ldots$ & -11.303 & $\ldots$ & -11.729 & -12.100 & -12.373 \\
\hline $2135-147 \ldots \ldots$ & -11.935 & $\ldots$ & -12.233 & $<-13.145$ & $<-12.295$ \\
\hline $2304+042 \ldots \ldots$ & $\ldots$ & $\ldots$ & $\ldots$ & $\ldots$ & $\ldots$ \\
\hline
\end{tabular}

a The fluxes are in ergs $\mathrm{cm}^{-2} \mathrm{~s}^{-1}$.

\subsection{Equivalent Width-Continuum Luminosity Correlations}

\subsubsection{UV Lines}

The best known line-continuum correlation for quasars is the inverse correlation between the emission-line EWs and the continuum, commonly known as the Baldwin effect. It was detected for the first time by Baldwin (1977) in a sample of high-redshift quasars in which the EW of the C IV 21549 line decreased with increasing UV continuum luminosity, $L(1450 \AA)$. The Baldwin effect has since been reported for a large number of lines (O vI, $\mathrm{N} \mathrm{v}, \mathrm{He}$ II, $\mathrm{C}$ III], $\mathrm{Mg}$ II, and Ly $\alpha$ ) in a number of different samples (e.g., Tytler \& Fan 1992; Zamorani et al. 1992; Green 1996). Baldwin et al. (1978) showed, however, that the correlation is much stronger in a sample of flat-spectrum RLQs. This was later confirmed by Wampler et al. (1984) and Baldwin, Wampler, \& Gaskell (1989). On the other hand, the analysis of optically selected quasar samples (Osmer 1980; Crampton, Cowley, \& Hartwick 1990; Boyle, Jones, \& Shanks 1991) shows that the Baldwin effect is less pronounced. It has been suggested that there exist two kinds of the Baldwin effect: a global, or object-to-object, effect and an intrinsic effect due to variability of the object (Pogge \& Peterson 1992; Murdoch 1983). The slope of the intrinsic effect is different from that of the global one, adding scatter to the global relationship. However, as the result of variability and selection effects, some regions of the EW- $L$ diagram may be left unpopulated and hence result in a more pronounced Baldwin effect for the RLQs.

The correlation results between EW and continuum luminosity are presented in Table 13, with coding explained in $\S 3$. The primary correlations are italicized and are displayed for the Ly $\alpha, \mathrm{H} \beta$, and $\mathrm{C}$ III] lines in Figures $4 a, 4 b$, and $4 c$.

The Baldwin effect is present in our sample for the Ly $\alpha$ line, for which the EW anticorrelates with all the various optical and UV (OUV) luminosities. The primary correlation is with $L(0.1-0.2 \mu \mathrm{m})$ luminosity.

The EW(C III]) shows a very strong (primary) correlation 
TABLE 5

OPTICAL LINE FLUXES ${ }^{\mathrm{a}}$

\begin{tabular}{|c|c|c|c|c|c|c|c|}
\hline Name & $\log F(\mathrm{H} \beta)$ & $\log F([\mathrm{O} \mathrm{III}])$ & $\log F(\mathrm{Fe}$ II $)$ & $\log F(\mathrm{H} \gamma)$ & $\log F(\mathrm{H} \delta)$ & $\log F([\mathrm{O} \mathrm{II}])$ & $\log F(\mathrm{Mg}$ II $)$ \\
\hline $0007+106 \ldots \ldots$ & -12.338 & -12.660 & -13.088 & -12.813 & -13.020 & -13.477 & $\ldots$ \\
\hline $0026+129 \ldots \ldots$ & -12.223 & -12.605 & $<-13.413$ & -12.821 & -13.131 & $<-13.852$ & $\ldots$ \\
\hline $0044+030 \ldots \ldots$ & $\ldots$ & $\ldots$ & $\ldots$ & $\ldots$ & $\ldots$ & $<-14.000$ & -12.843 \\
\hline $0050+124 \ldots \ldots$ & -12.267 & -12.894 & -12.327 & -12.812 & $<-13.219$ & $<-13.677$ & $\ldots$ \\
\hline $0054+145 \ldots \ldots$ & -12.849 & -13.428 & $<-13.860$ & -13.570 & $<-14.200$ & $<-14.435$ & -12.643 \\
\hline $0133+207 \ldots \ldots$ & $\ldots$ & $\ldots$ & $\ldots$ & $\ldots$ & 13.680 & $<-14.254$ & -12.256 \\
\hline $0134+329 \ldots \ldots$ & $\ldots$ & $\ldots$ & 13.553 & -13.277 & -13.810 & -13.560 & -12.685 \\
\hline $0205+024 \ldots \ldots$ & -12.793 & -13.085 & -13.307 & -13.097 & -13.496 & $<-13.918$ & $\ldots$ \\
\hline $0312-770 \ldots \ldots$ & $<-12.890$ & -13.749 & -13.237 & $<-13.066$ & $\ldots$ & $\ldots$ & $\ldots$ \\
\hline $0637-752 \ldots \ldots$ & -12.714 & -13.272 & $<-13.405$ & $<-13.145$ & $<-13.514$ & $<-13.901$ & $\ldots$ \\
\hline $0804+761 \ldots \ldots$ & -11.750 & -12.446 & -12.588 & -12.334 & -12.664 & $<-13.166$ & $\ldots$ \\
\hline $0837-120 \ldots \ldots$ & -12.907 & -13.077 & $<-13.903$ & -13.454 & $<-13.973$ & $\ldots$ & $\ldots$ \\
\hline $0844+349 \ldots \ldots$ & -12.513 & -13.083 & -12.647 & -12.809 & -13.156 & $<-13.798$ & $\ldots$ \\
\hline $0903+169 \ldots \ldots$ & $\ldots$ & $\ldots$ & $\ldots$ & -13.852 & $<-14.417$ & -14.845 & -13.098 \\
\hline $0915+165 \ldots \ldots$ & $\ldots$ & $\ldots$ & $\ldots$ & $\ldots$ & $\ldots$ & $\ldots$ & $\ldots$ \\
\hline $0923+392 \ldots \ldots$ & $\ldots$ & $\ldots$ & $\ldots$ & $\ldots$ & $\ldots$ & $<-13.682$ & -12.609 \\
\hline $1001+054 \ldots \ldots$ & -12.588 & -13.432 & -13.749 & -13.244 & -13.763 & $<-14.337$ & $\ldots$ \\
\hline $1004+130 \ldots \ldots$ & $<-13.000$ & $<-13.003$ & $<-12.650$ & -12.570 & $<-13.116$ & $<-13.256$ & $<-12.656$ \\
\hline $1012+008 \ldots \ldots$ & -12.927 & -13.324 & -13.472 & -13.288 & -13.713 & -14.148 & -13.527 \\
\hline $1028+313 \ldots \ldots$ & -12.904 & -13.406 & $<-13.855$ & -13.440 & -13.711 & $<-14.321$ & -12.551 \\
\hline $1100+772 \ldots \ldots$ & $\ldots$ & $\ldots$ & $<-13.572$ & -13.122 & -13.637 & -14.048 & -12.280 \\
\hline $1116+215 \ldots \ldots$ & -12.478 & -12.951 & -13.081 & -12.796 & -13.140 & $<-13.920$ & -12.050 \\
\hline $1121+422 \ldots \ldots$ & -12.720 & -13.736 & -13.719 & -13.247 & -13.534 & $<-14.302$ & -13.074 \\
\hline $1137+661 \ldots \ldots$ & $\ldots$ & $\ldots$ & $\ldots$ & $\ldots$ & $\ldots$ & $<-13.316$ & -12.441 \\
\hline $1202+281 \ldots \ldots$ & -13.828 & -14.312 & $<-14.182$ & -14.107 & $<-14.332$ & $<-14.615$ & -13.420 \\
\hline $1211+143 \ldots \ldots$ & -12.217 & -12.884 & -12.556 & -12.521 & -12.697 & $<-13.639$ & $\ldots$ \\
\hline $1217+023 \ldots \ldots$ & -12.408 & -12.713 & $<-13.284$ & -12.733 & -13.202 & $<-13.670$ & -12.100 \\
\hline $1226+023 \ldots \ldots$ & -11.609 & -12.378 & -12.236 & -12.160 & -12.532 & $<-12.898$ & $\ldots$ \\
\hline $1307+085 \ldots \ldots$ & -12.542 & -12.992 & -13.641 & -12.710 & -13.328 & $<-13.914$ & $\ldots$ \\
\hline $1351+640 \ldots \ldots$ & -12.573 & -12.596 & $<-13.119$ & -12.845 & -12.969 & $<-13.414$ & $\ldots$ \\
\hline $1407+265 \ldots \ldots$ & $\ldots$ & $\ldots$ & $\ldots$ & $\ldots$ & $\ldots$ & $\ldots$ & $\ldots$ \\
\hline $1416-129 \ldots \ldots$ & $\ldots$ & -13.236 & $<-14.148$ & -13.318 & $\ldots$ & $\ldots$ & $\ldots$ \\
\hline $1426+015 \ldots \ldots$ & -12.480 & -12.937 & -13.117 & -12.807 & -13.019 & $<-13.824$ & $\ldots$ \\
\hline $1501+106 \ldots \ldots$ & -12.397 & -12.519 & -13.139 & -12.758 & -13.189 & -13.599 & $\cdots$ \\
\hline $1613+658 \ldots \ldots$ & -12.515 & -13.008 & -13.404 & -13.075 & -13.715 & -13.881 & $\begin{array}{l}\cdots \\
\cdots\end{array}$ \\
\hline $1704+608 \ldots \ldots$ & -13.171 & -12.953 & $<-13.944$ & -13.593 & -13.812 & $<-13.997$ & -12.694 \\
\hline $1721+343 \ldots \ldots$ & -12.393 & -12.504 & $<-13.128$ & -12.591 & -13.057 & $<-13.596$ & -12.801 \\
\hline $1803+676 \ldots \ldots$ & -12.824 & -13.211 & $<-13.912$ & -13.003 & -13.888 & $<-14.229$ & $\ldots$ \\
\hline $2130+099 \ldots \ldots$ & -12.390 & -12.888 & -12.659 & -12.793 & -13.130 & $<-13.796$ & $\ldots$ \\
\hline $2135-147 \ldots \ldots$ & -12.950 & -12.966 & $<-14.225$ & -13.258 & -13.995 & $<-13.528$ & $\ldots$ \\
\hline $2304+042 \ldots \ldots$ & -12.347 & -12.579 & $<-13.504$ & -12.795 & -13.107 & -13.645 & $\ldots$ \\
\hline
\end{tabular}

${ }^{a}$ The fluxes are in ergs $\mathrm{cm}^{-2} \mathrm{~s}^{-1}$.

with $L(1-2 \mathrm{keV})$ and a marginal correlation with $L(0.1-0.2$ $\mu \mathrm{m})$. A C III]-X-ray correlation was reported by Green (1996) in a sample of quasars observed by the Einstein and IUE satellites. However, our C III] line sample has more than $50 \%$ upper limits, the correlation looks unconvincing (Fig. 4b), and no related correlation between the C III] flux and X-ray flux is seen ( $\S 3.2$ ). This suggests that the $\mathrm{EW}(\mathrm{C} \mathrm{III])} \mathrm{versus} L(1-2 \mathrm{keV})$ correlation is spurious, and we cannot confirm the correlation reported by Green (1996).

The traditional Baldwin effect for the $\mathrm{C}$ IV line is not present in our sample (Fig. 5a). However, a significant correlation $(P<1 \%$ in all cases) is found for both $C$ IV and $\mathrm{C}$ III] lines if we omit seven objects with low $\log v L_{v}(0.1-$ $0.2 \mu$ ), i.e., I $\mathrm{Zw} 1$, NAB $0205+024$, PG $0844+349$, PG $1012+008$, PG $1211+143$, PG 1351+640, and II Zw 136. These objects have relatively weak carbon lines [EW(C Iv)/ $\mathrm{EW}(\mathrm{Ly} \alpha)<0.55]$, as can be seen in Figures $5 a, 5 b$, and $5 c$, where they are indicated by filled circles. They also have narrow FWHM of $\mathrm{H} \beta$ (less than $2500 \mathrm{~km} \mathrm{~s}^{-1}$; Table 7) falling into the range of narrow-line Seyfert 1 (Sy1) objects (NLSy1; Osterbrock \& Pogge 1985; except for PG $0844+349$ and PG $1351+640$, which are broad absorption line QSOs). This suggests that NLSy1s may have a systematically lower carbon-line EW and so do not follow the general Baldwin effect for the rest of the sample.

The only significant correlation involving $\alpha_{o x}$ is a strong anticorrelation with $\mathrm{EW}(\mathrm{C}$ IV) (Fig. 6a). A marginal correlation with $\mathrm{EW}(\mathrm{H} \beta)$ becomes significant when one object, PG $1001+054$, is omitted (Table 13). For the O vI line, marginal anticorrelations of $\mathrm{EW}\left(\mathrm{O}\right.$ vI) with $L_{\mathrm{opt}}\left(P_{K}=\right.$ $\left.1.7 \%, P_{S}=3.8 \%\right)$, and with $\alpha_{o x}\left(P_{K}=3.1 \%, P_{S}=4.1 \%\right)$ are present in our sample. The latter correlation was reported by Zheng, Kriss, \& Davidsen (1995) in their sample of 30 QSOs and two Sy1s $(z>0.15)$, which had both $I U E$ and Einstein X-ray fluxes. However, with only 10 points in our sample, we cannot draw strong conclusions from our lack of a correlation here. 
TABLE 6

REST Frame UV LINE WIDTHS ${ }^{\mathrm{a}}$

\begin{tabular}{|c|c|c|c|c|c|}
\hline \multirow[b]{2}{*}{ NAME } & \multicolumn{5}{|c|}{ FWHM } \\
\hline & $\operatorname{Ly} \alpha \mathrm{N} \mathrm{v}$ & $\operatorname{Ly} \beta$ O vI & $\mathrm{C}$ IV & Si IV O IV] & $\mathrm{Al}$ III C III] \\
\hline $0007+106 \ldots \ldots$ & 3777.78 & $\ldots$ & 3660.42 & 8756.05 & $<3665.08$ \\
\hline $0026+129 \ldots \ldots$ & 1975.31 & $\ldots$ & 2734.66 & 4680.60 & $\ldots$ \\
\hline $0044+030 \ldots \ldots$ & 10764.71 & 4467.18 & 5364.74 & $\ldots$ & 4496.84 \\
\hline $0050+124 \ldots \ldots$ & 2629.63 & $\ldots$ & 5581.07 & 5337.08 & $<11324.01$ \\
\hline $0054+145 \ldots \ldots$ & 11780.27 & $\ldots$ & 7669.45 & $\ldots$ & $\ldots$ \\
\hline $0133+207 \ldots \ldots$ & $\ldots$ & $\ldots$ & $\ldots$ & $\ldots$ & $\ldots$ \\
\hline $0134+329 \ldots \ldots$ & $<7550.88$ & $<3572.99$ & $<4469.97$ & $\ldots$ & $<2827.03$ \\
\hline $0205+024 \ldots \ldots$ & 1627.16 & $\ldots$ & 2519.69 & $<4498.93$ & $<5942.90$ \\
\hline $0312-770 \ldots \ldots$ & 3074.08 & 6935.13 & 4491.28 & $\ldots$ & $\ldots$ \\
\hline $0637-752 \ldots \ldots$ & 2985.19 & 6592.44 & 2829.56 & $\ldots$ & $<2985.36$ \\
\hline $0804+761 \ldots \ldots$ & 4498.77 & $\ldots$ & 4038.08 & 4810.31 & 11507.97 \\
\hline $0837-120 \ldots \ldots$ & 5572.85 & $\ldots$ & 3960.61 & $\ldots$ & $\ldots$ \\
\hline $0844+349 \ldots \ldots$ & 4054.33 & $\ldots$ & 4289.86 & 6719.19 & $<7603.27$ \\
\hline $0903+169 \ldots \ldots$ & $\ldots$ & $\ldots$ & $\ldots$ & $\ldots$ & $\ldots$ \\
\hline $0915+165 \ldots \ldots$ & $\ldots$ & $\ldots$ & $\ldots$ & $\ldots$ & $\ldots$ \\
\hline $0923+392 \ldots \ldots$ & $\ldots$ & $\ldots$ & $\ldots$ & $\ldots$ & $\ldots$ \\
\hline $1001+054 \ldots \ldots$ & $\ldots$ & $\ldots$ & $\ldots$ & $\begin{array}{l}\cdots \\
\cdots\end{array}$ & $\begin{array}{l}\cdots \\
\cdots\end{array}$ \\
\hline $1004+130 \ldots \ldots$ & 11884.96 & $\ldots$ & $\ldots$ & $<11703.44$ & $\ldots$ \\
\hline $1012+008 \ldots \ldots$ & 4822.23 & $\ldots$ & 5697.86 & 3100.93 & $\ldots$ \\
\hline $1028+313 \ldots \ldots$ & 3454.33 & $\ldots$ & 3424.14 & $<4767.79$ & $<4886.77$ \\
\hline $1100+772 \ldots \ldots$ & 3753.09 & $<7980.63$ & 7772.10 & $<4171.66$ & $<9246.97$ \\
\hline $1116+215 \ldots \ldots$ & 3241.98 & $\ldots$ & 6161.90 & $<5097.00$ & 10209.39 \\
\hline $1121+422 \ldots \ldots$ & $\ldots$ & $\ldots$ & $\ldots$ & $\ldots$ & $\ldots$ \\
\hline $1137+661 \ldots \ldots$ & 4355.56 & $<7709.96$ & 3710.77 & $<11596.07$ & 3146.21 \\
\hline $1202+281 \ldots \ldots$ & 4017.29 & $\ldots$ & 3780.50 & 3665.71 & $<5551.86$ \\
\hline $1211+143 \ldots \ldots$ & 2076.55 & $\ldots$ & 2733.50 & 7985.76 & 4436.14 \\
\hline $1217+023 \ldots \ldots$ & 4037.04 & 3868.34 & 3788.24 & 5274.16 & 6245.26 \\
\hline $1226+023 \ldots \ldots$ & 3634.57 & $\ldots$ & 4522.26 & 3708.66 & 4528.91 \\
\hline $1307+085 \ldots \ldots$ & 3807.41 & $\ldots$ & 4545.51 & 5074.45 & $\ldots$ \\
\hline $1351+640 \ldots \ldots$ & 2370.37 & $\ldots$ & 2112.97 & 7193.99 & 6025.60 \\
\hline $1407+265 \ldots \ldots$ & $\ldots$ & $\ldots$ & $\ldots$ & $\ldots$ & $\ldots$ \\
\hline $1416-129 \ldots \ldots$ & 5767.66 & $\ldots$ & 7355.70 & $<8199.00$ & $\ldots$ \\
\hline $1426+015 \ldots \ldots$ & 5234.58 & $\ldots$ & 4245.31 & 10146.32 & 5834.41 \\
\hline $1501+106 \ldots \ldots$ & $\ldots$ & $\ldots$ & $\ldots$ & $\ldots$ & $\ldots$ \\
\hline $1613+658 \ldots \ldots$ & 8203.47 & $\ldots$ & 8104.44 & $<6270.58$ & $<8394.62$ \\
\hline $1704+608 \ldots \ldots$ & 6251.86 & $\ldots$ & 2424.79 & $<2235.08$ & 4613.19 \\
\hline $1721+343 \ldots \ldots$ & 3446.92 & 3818.97 & 4208.51 & 7342.17 & $<9141.15$ \\
\hline $1803+676 \ldots \ldots$ & 6637.54 & $\ldots$ & 3648.80 & 9098.79 & $<6982.36$ \\
\hline $2130+099 \ldots \ldots$ & 2133.34 & $\ldots$ & 2362.81 & 4464.57 & 3908.32 \\
\hline $2135-147 \ldots \ldots$ & 7430.14 & $\ldots$ & 8140.08 & $<5499.43$ & $<11830.29$ \\
\hline $2304+042 \ldots \ldots$ & $\ldots$ & $\ldots$ & $\ldots$ & $\ldots$ & $\ldots$ \\
\hline
\end{tabular}

${ }^{\mathrm{a}}$ FWHMs are in $\mathrm{km} \mathrm{s}^{-1}$.

\subsubsection{Balmer Lines}

The primary correlation for $\operatorname{EW}(\mathrm{H} \beta)$ is with $L(0.4-0.8$ $\mu \mathrm{m})($ Fig. $4 c)$. The $\mathrm{EW}(\mathrm{H} \beta)$ also anticorrelates with the $L(100-10 \mu \mathrm{m})$ IR luminosity, $L(0.1-0.2 \mu \mathrm{m})$, OUV luminosities, and X-ray $L(1-10 \mathrm{keV})$ luminosity (Table 13$)$. A closer look shows that the "correlation" between $\operatorname{EW}(\mathrm{H} \beta)$ and $L(1-10 \mathrm{keV})$ is due to a systematically higher $L(1-10$ $\mathrm{keV}$ ) for RLQs (Fig. 4d). The $L(1-10 \mathrm{keV}$ ) luminosities are determined by extrapolating the Einstein soft X-ray (0.1-3.5 $\mathrm{keV}$ ) flux and energy index. The systematically flatter $\alpha_{\mathrm{x}}$ and stronger X-ray flux of the RLQs (Wilkes \& Elvis 1987) exaggerates the systematic difference between $L(1-10 \mathrm{keV})$ in the two classes.

Similar to $\mathrm{H} \beta$, the EWs of the other Balmer lines anticorrelate with the far-IR luminosity, $L(10-100 \mu \mathrm{m})$.

\subsubsection{Fe II Line}

No significant correlations are found for the EW of the
Fe II $\lambda 4570$ optical multiplet. The one with the lowest probability of chance occurrence $\left(P_{K}=2.1 \%, P_{S}=3.5 \%\right)$ is an anticorrelation with $L(1-2 \mathrm{keV})$ (reported as significant by Boroson \& Green 1992 and others). However, Figure $7 a$ does show a trend for objects with larger $L(1-2 \mathrm{keV})$ luminosity and smaller EW (Fe II) to be RLQs, while radio-quiet objects have a much larger range in EW (Fe II) and concentrate toward smaller X-ray luminosities.

No correlation between the EW (Fe II) line and $\alpha_{\mathrm{x}}$ is present in our sample (see Fig. 7b), nor in the RQQ and RLQ subsamples. Such correlations have been found by Wilkes, Elvis, \& McHardy (1987) and Shastri et al. (1993) in samples observed by the Einstein satellite. However, Boroson (1989), studying a radio-selected sample, Walter \& Fink (1993), who studied the Seyfert galaxies observed in the ROSAT All Sky Survey, and Zheng \& O'Brien (1990) failed to confirm this correlation. Thirty-one objects in our sample are also included in the Shastri et al. (1993) sample; 
TABLE 7

Rest Frame Optical Line Widths ${ }^{\mathrm{a}}$

\begin{tabular}{|c|c|c|c|c|c|c|c|c|c|}
\hline \multirow[b]{2}{*}{ NAME } & \multicolumn{9}{|c|}{ FWHM } \\
\hline & $\mathrm{H} \beta$ & {$[\mathrm{O} \mathrm{III}]$} & $\mathrm{H} \gamma$ & $\mathrm{H} \delta$ & {$[\mathrm{Ne}$ III] } & $\mathrm{NeHe}$ & {$[\mathrm{O} \mathrm{II}]$} & $\mathrm{Ne} v$ & Mg II \\
\hline $0007+106 \ldots \ldots$ & 3840.59 & 527.92 & 3994.68 & 3712.33 & 2973.24 & 617.21 & 504.54 & 2300.36 & $\ldots$ \\
\hline $0026+129 \ldots \ldots$ & 1518.21 & 395.45 & 2968.88 & 2847.15 & 459.68 & 471.44 & $\ldots$ & 507.88 & $\ldots$ \\
\hline $0044+030 \ldots \ldots$ & $\ldots$ & $\ldots$ & $\ldots$ & $\ldots$ & $\ldots$ & $\ldots$ & $\ldots$ & $\ldots$ & 6374.92 \\
\hline $0050+124 \ldots \ldots$ & 2589.60 & $<2193.52$ & 6121.62 & 4304.73 & 4538.57 & $\ldots$ & $\ldots$ & $\ldots$ & $\ldots$ \\
\hline $0054+145 \ldots \ldots$ & 4895.93 & 970.04 & 4087.99 & 1386.64 & 656.25 & 692.42 & $\ldots$ & 934.33 & 7447.43 \\
\hline $0133+207 \ldots \ldots$ & $\ldots$ & $\ldots$ & 1216.58 & 1772.79 & 870.97 & $<1577.14$ & 580.36 & 2758.33 & 12130.76 \\
\hline $0134+329 \ldots \ldots$ & $\ldots$ & $\ldots$ & 3781.77 & 2695.76 & 2880.78 & 1635.30 & 1138.67 & 1162.53 & 4370.25 \\
\hline $0205+024 \ldots \ldots$ & 1579.31 & 906.53 & 2554.13 & 1980.50 & 1940.02 & 932.79 & $\ldots$ & $\ldots$ & $\ldots$ \\
\hline $0312-770 \ldots \ldots$ & 3185.16 & 813.66 & 2839.61 & $\ldots$ & $\ldots$ & $\ldots$ & $\ldots$ & $\ldots$ & $\ldots$ \\
\hline $0637-752 \ldots \ldots$ & 3860.34 & 1011.56 & 4312.65 & 3800.09 & 4324.61 & 2119.92 & $\ldots$ & 1215.42 & $\ldots$ \\
\hline $0804+761 \ldots \ldots$ & 3454.24 & 799.88 & 3465.19 & 2146.51 & 1305.70 & 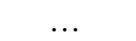 & 429.84 & $<1598.96$ & $\ldots$ \\
\hline $0837-120 \ldots \ldots$ & 4038.08 & 1499.64 & 3944.49 & 3202.58 & 3247.23 & $<1645.38$ & $\ldots$ & $\ldots$ & $\ldots$ \\
\hline $0844+349 \ldots \ldots$ & 2555.54 & 534.45 & 3107.82 & 1951.24 & 2302.93 & 739.72 & 560.24 & $<534.15$ & $\ldots$ \\
\hline $0903+169 \ldots \ldots$ & $\ldots$ & $\ldots$ & 2006.67 & 3311.55 & $<2008.37$ & 673.04 & 668.10 & 848.51 & 6905.65 \\
\hline $0915+165 \ldots \ldots$ & $<2950.02$ & $<1000.60$ & $\ldots$ & $\ldots$ & $\ldots$ & $\ldots$ & $\ldots$ & $\ldots$ & $\ldots$ \\
\hline $0923+392 \ldots \ldots$ & $\ldots$ & $\ldots$ & $\ldots$ & $\ldots$ & $\ldots$ & $\ldots$ & $\ldots$ & $\ldots$ & 5950.12 \\
\hline $1001+054 \ldots \ldots$ & 2516.16 & 1026.96 & 1826.26 & 1932.23 & 1442.54 & 459.03 & $\ldots$ & 578.81 & 1475.33 \\
\hline $1004+130 \ldots \ldots$ & 4728.06 & 1185.14 & 4188.64 & $<2932.71$ & $\ldots$ & $\ldots$ & $\ldots$ & $\ldots$ & $\ldots$ \\
\hline $1012+008 \ldots \ldots$ & 2112.54 & 1210.30 & 2732.47 & 2272.30 & 2311.24 & 517.96 & 590.83 & $<3679.44$ & 3439.59 \\
\hline $1028+313 \ldots \ldots$ & 5882.15 & 769.32 & 4717.71 & 4719.40 & 945.06 & 1052.98 & 410.52 & 1501.76 & 7347.18 \\
\hline $1100+772 \ldots \ldots$ & $\ldots$ & $\ldots$ & 4622.32 & 1697.46 & 2801.92 & 690.87 & 445.13 & 1876.54 & 5528.21 \\
\hline $1116+215 \ldots \ldots$ & 3086.42 & 1455.36 & 2771.87 & 3225.25 & 2639.37 & $<2231.57$ & $\ldots$ & $\ldots$ & 3853.45 \\
\hline $1121+422 \ldots \ldots$ & 2985.82 & 693.23 & 3732.07 & 2881.74 & 1787.30 & $<2677.42$ & $\ldots$ & 1151.49 & 3593.98 \\
\hline $1137+661 \ldots \ldots$ & $\ldots$ & $\ldots$ & $\ldots$ & $\ldots$ & $\ldots$ & $\ldots$ & $\ldots$ & $\ldots$ & 2864.89 \\
\hline $1202+281 \ldots \ldots$ & 3306.13 & 517.08 & 4390.07 & 5752.07 & $<1179.44$ & $<2341.68$ & $\ldots$ & $\ldots$ & 6458.87 \\
\hline $1211+143 \ldots \ldots$ & 1845.93 & 1118.03 & 2662.66 & 2594.83 & 1698.47 & $<2780.55$ & $\ldots$ & 1435.21 & $\ldots$ \\
\hline $1217+023 \ldots \ldots$ & 2123.65 & 624.32 & 4001.52 & 3768.65 & $<3411.52$ & 655.98 & $\ldots$ & 855.52 & 3848.09 \\
\hline $1226+023 \ldots \ldots$ & 4364.56 & $<2172.01$ & 4150.20 & 3426.37 & 3325.11 & $\ldots$ & $\ldots$ & 3001.94 & $\ldots$ \\
\hline $1307+085 \ldots \ldots$ & 5384.10 & 964.05 & 5030.15 & 4179.67 & $<2770.77$ & 1017.31 & 599.68 & $<1352.46$ & $<4290.90$ \\
\hline $1351+640 \ldots \ldots$ & 855.38 & 775.31 & 2056.44 & 4781.57 & 1517.39 & $<2692.15$ & 587.61 & $\ldots$ & $\ldots$ \\
\hline $1407+265 \ldots \ldots$ & $\ldots$ & $\ldots$ & $\ldots$ & $\ldots$ & $\ldots$ & $\ldots$ & $\ldots$ & $\ldots$ & 7611.48 \\
\hline $1416-129 \ldots \ldots$ & 4360.24 & 1097.66 & 4105.27 & 1906.63 & 1054.69 & 1413.54 & 567.64 & 2551.24 & $\ldots$ \\
\hline $1426+015 \ldots \ldots$ & 5835.25 & 1257.64 & 4677.62 & 5723.55 & 1601.31 & 1170.84 & $\ldots$ & 1023.65 & $\ldots$ \\
\hline $1501+106 \ldots \ldots$ & 1865.67 & 418.21 & 4409.28 & 2477.08 & 719.76 & 593.95 & 436.28 & 686.52 & $\ldots$ \\
\hline $1613+658 \ldots \ldots$ & 6997.36 & 1270.22 & 5177.39 & 3983.66 & 3720.52 & 606.35 & 1000.54 & 2990.99 & $\ldots$ \\
\hline $1704+608 \ldots \ldots$ & 1209.02 & 977.83 & 3116.11 & $<2320.28$ & 2269.66 & 2273.44 & 426.62 & 1811.04 & 7817.77 \\
\hline $1721+343 \ldots \ldots$ & 2400.75 & 699.82 & 3787.72 & 1334.71 & 1010.08 & 968.46 & 668.10 & 639.23 & 3716.21 \\
\hline $1803+676 \ldots \ldots$ & 2848.19 & 948.89 & 4054.81 & 1582.64 & 873.24 & 505.55 & 459.62 & 1884.42 & $\ldots$ \\
\hline $2130+099 \ldots \ldots$ & 2058.85 & 608.15 & 2664.73 & 2138.47 & 2207.67 & 714.91 & 610.95 & 2621.03 & $\ldots$ \\
\hline $2135-147 \ldots \ldots$ & 2511.84 & 827.50 & 4255.55 & 1191.37 & 1168.10 & 999.48 & 760.67 & $\ldots$ & 4185.83 \\
\hline $2304+042 \ldots \ldots$ & 4093.01 & 620.73 & 3325.69 & 4333.25 & 1209.68 & 894.02 & 474.91 & 718.92 & $\ldots$ \\
\hline
\end{tabular}

${ }^{\mathrm{a}}$ FWHMs are in $\mathrm{km} \mathrm{s}^{-1}$.

however, only 16 of them have Fe II measurements. A comparison of our Figure $7 b$ with their Figure $5 a$ shows small disagreements between the measured values and one incorrect slope. PG $0844+349$ has an X-ray energy index $\alpha_{\mathrm{x}}$ of 1.6 in Shastri et al. (1993), whereas the correct value from the Einstein data is 0.6 (E94).

Given the apparently discrepant results in the literature, we conclude (as did Lawrence et al. 1997) that there is no direct correlation between Fe II and $\alpha_{x}$, but rather a zone of avoidance such that objects with flat X-ray spectra do not have strong Fe II. A similar effect, that objects with stronger $\mathrm{X}$-ray luminosities do not have strong $\mathrm{Fe}$ II, would also explain our marginal correlation between Fe II and $L(1-2$ $\mathrm{keV}$ ) (Fig. 7a). This also agrees with the long-established tendency toward weak Fe II in RLQs (Peterson, Foltz, \& Byard 1981; Phillips 1977; Osterbrock 1977).

Lawrence et al. (1997) note a much stronger correlation between $\mathrm{Fe}$ iI and the near-IR-X-ray slope $\alpha_{\mathrm{ix}}$. We tested for this in our sample and found no significant correlation $\left(P_{K}=5.0 \%, P_{S}=6.9 \%\right)$. However, our data cover only about $60 \%$ of the range in $\alpha_{\mathrm{ix}}$ and a smaller range in $\log (\mathrm{Fe}$ II $/ \mathrm{H} \beta)$ than that of Lawrence et al. $(1997 ;-1.5$ to 0 in our sample; see -1 to 0.7 in theirs).

\subsection{Line Flux versus Continuum Flux Correlations}

The OUV line flux-continuum flux correlations are presented in Table 14, with similar coding to Table 13. This analysis was performed identically in both flux and luminosity space to guard against purely redshift-induced results. However, given the small range in redshift $(z<0.4$ for all but five sources; Fig. 1), there is no significant difference between the two sets of results. All the primary correlations for these lines are shown in Figure 8. For Ly $\alpha$ and $\mathrm{C}$ IV lines the primary correlations are with the continuum regions closest to the driving continuum, as is expected. However, we note that simultaneous measurements of both 
TABLE 8

SED DETAILS FOR OBJeCtS Not INCLUDED IN E94 ${ }^{\mathrm{a}}$

\begin{tabular}{|c|c|c|c|c|c|c|c|c|}
\hline Name & Radio Data & Far-IR Data & Optical Data & UV Data & X-Ray Data & $E(B-V)$ & Starlight $\log v L_{v \mathrm{H}}$ & Starlight Reference \\
\hline $0044+030 \ldots \ldots$ & 1 & 12 & 15,16 & $24^{\mathrm{b}}$ & 26 & 0.06 & $45.30_{-0.15}^{+0.15}$ & 31 \\
\hline $0133+207 \ldots \ldots$ & $2,3,4,5$ & 13 & 17,18 & 25 & 30 & 0.056 & $45.10_{-0.15}^{+0.15}$ & 31 \\
\hline $0903+169 \ldots \ldots$ & $4,5,6$ & 13 & 15 & $25^{\mathrm{c}}$ & 26,27 & 0.076 & $44.42_{-0.12}^{+0.12}$ & 32 \\
\hline $0923+392 \ldots \ldots$ & $5,6,7,8$ & 14 & 15,19 & $25^{\mathrm{d}}$ & 27 & 0.032 & $45.60_{-0.15}^{+0.15}$ & 31 \\
\hline $1001+054 \ldots \ldots$ & 1,9 & 14 & $15,19,20$ & $\ldots$ & 26 & 0.04 & $44.20_{-0.12}^{+0.12}$ & 34 \\
\hline $1004+130 \ldots \ldots$ & $\ldots$ & 12,13 & $16,20,21,22,23$ & 24 & 26 & 0.07 & $44.80_{-0.12}^{+0.12}$ & 33 \\
\hline $1012+008 \ldots \ldots$ & 1 & 12 & 16,17 & 24 & $\ldots$ & 0.065 & $44.91_{-0.12}^{+0.12}$ & 33 \\
\hline $1121+422 \ldots \ldots$ & 1 & 12 & $15,16,17$ & 25 & $\ldots$ & 0.047 & $44.65_{-0.12}^{+0.12}$ & 33 \\
\hline $1217+023 \ldots \ldots$ & 7,10 & 13 & $15,17,20,23$ & 24 & $27,28,29$ & 0.039 & $44.58_{-0.12}^{+0.12}$ & 35 \\
\hline $1351+640 \ldots \ldots$ & 1,11 & 14 & 16,21 & 24 & 26 & 0.05 & $44.38_{-0.12}^{+0.11}$ & 34 \\
\hline $2304+042 \ldots \ldots$ & 1 & 13 & 15,17 & $\ldots$ & $\ldots$ & 0.110 & $43.66_{-0.15}^{+0.15}$ & 36 \\
\hline
\end{tabular}

a Elvis et al. 1994.

b The UV spectrum was deleted, as the difference between optical and UV spectra was 0.5 in $\log v L_{v}$.

${ }^{c}$ UV grayshifted by -0.193 in $v L_{v}$.

d UV grayshifted by -0.308 in $v L_{1}$.

REFERENCES.-Radio data: (1) Kellermann et al. 1989; (2) Bentley et al. 1975; (3) Swarup, Sinha, \& Hilldrup 1984; (4) Pooley \& Henbest 1974; (5) Steppe et al. 1988; (6) Owen et al. 1978; (7) Owen \& Puschell 1982; (8) Parley 1982; (9) Condon et al. 1981; (10) Miley \& Hartsuijker 1978; (11) Robson et al. 1985. Far-IR data: (12) Sanders et al. 1989; (13) E94; (14) Neugebauer et al. 1986. Optical and near-IR data: (15) this paper; (16) Neugebauer et al. 1987; (17) E94; (18) Penfold 1979; (19) Ennis \& Neugebauer 1982; (20) Hyland \& Allen 1982; (21) Neugebauer et al. 1979; (22) Sitko et al. 1982; (23) Cutri et al. 1985. UV data: (24) IUE data from Lanzetta, Turnshek, \& Sandoval 1993; (25) HST data; A. Dobrzycki 1998, private communication. X-ray data: (26) Wilkes et al. 1994; (27) Wilkes \& Elvis 1987; (28) Comastri et al. 1992; (29) Williams et al. 1992; (30) Wilkes et al. 1994. Starlight Reference: (31) $\frac{1}{3}$ total $L$; (32) Romanishin \& Hintzen 1989; (33) McLeod \& Rieke 1994a; (34) McLeod \& Rieke 1994b; (35) Taylor et al. 1996; (36) Smith et al. 1986.

PG0044+030 (PKS0044+030)

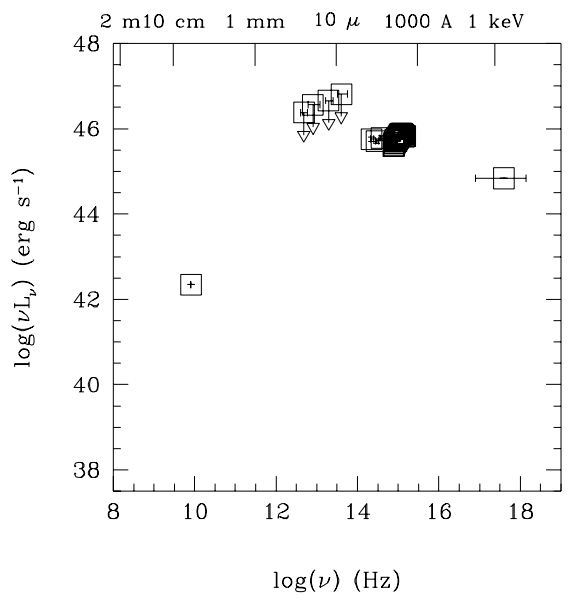

3C 215 (PKS0903+169)

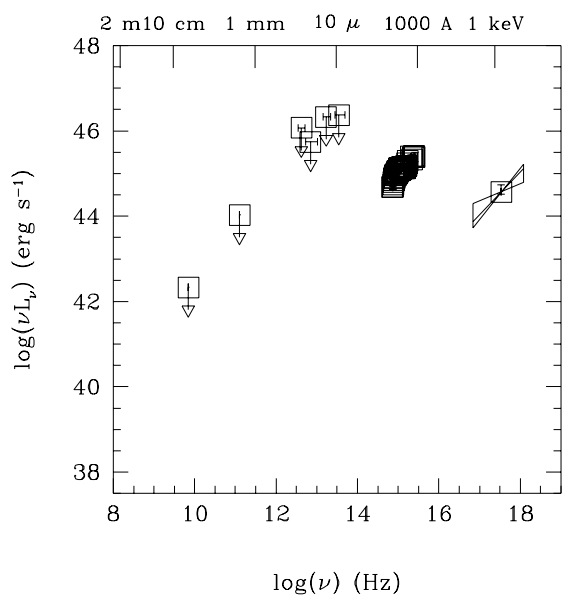

3C 47 (PKSO133+207)

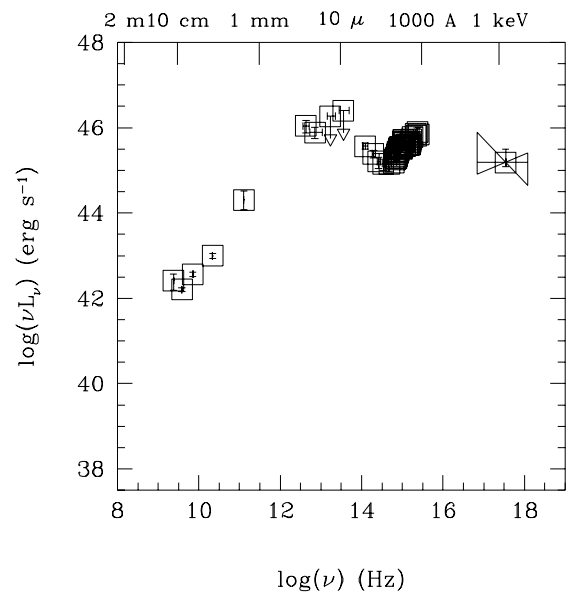

4C39.25 (B2 0923+392)

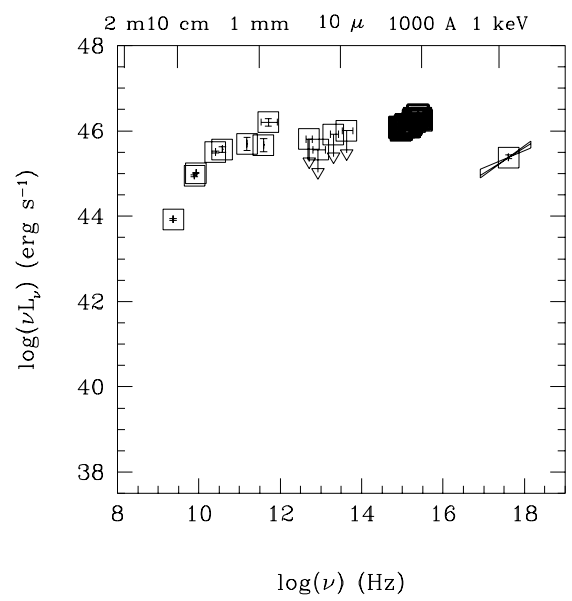

FIG. 3.-Radio-X-ray SEDs for the 11 new objects (Table 8) on a $\log v L_{v}$ vs. $\log v$ scale. 
PG1001+054

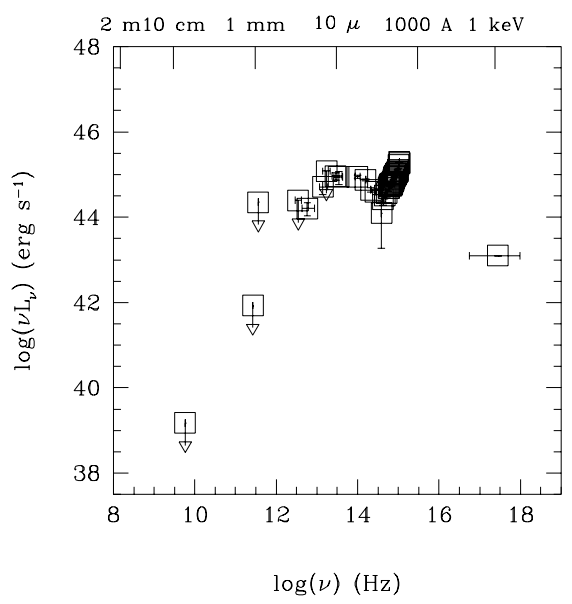

PG1012+008

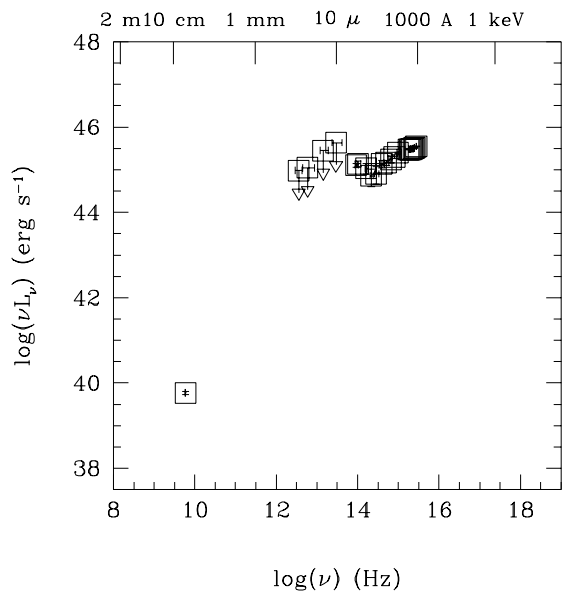

PG1004+130(PKS1004+130,4C 13.41)

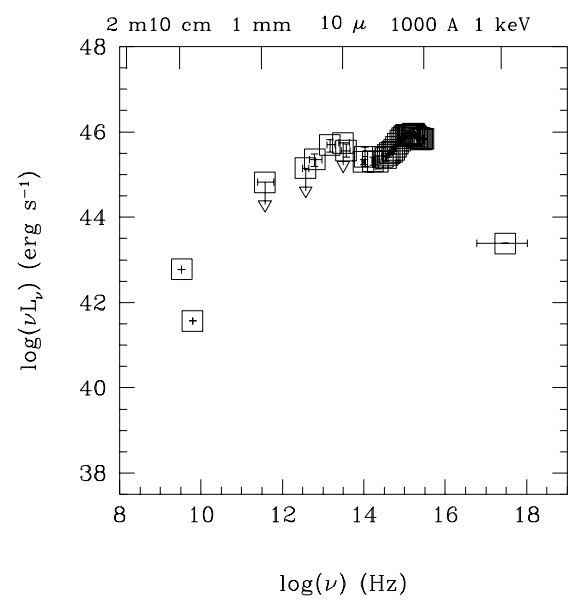

PG1 121+422

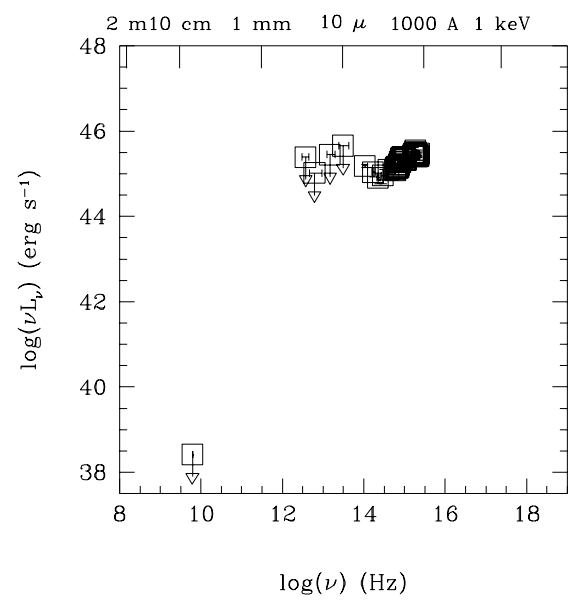

FIG. 3.-Continued

UV lines and continuum (as is the case in our sample) will result in smaller scatter and lower probabilities for these correlations. In other bands, small continuum variations or systematic shifts between the measurements will add scatter.

For the $\mathrm{C} \mathrm{III]} \mathrm{line} \mathrm{the} \mathrm{primary} \mathrm{correlation} \mathrm{is} \mathrm{with} \mathrm{the}$ optical flux, far from its driving continuum. No conclusions can be drawn for the Si IV line, as it is not clear what the driving continuum is for this line (T. Kallman 1997, private communication). Correlations for Ly $\alpha$ flux and EW are the most significant (usually $P \ll 0.1 \%$; compare $P \gg 0.3 \%$; see Table 14) and have the smallest scatter among the UV lines (Table 16). The predominance of the $\mathrm{EW}(\mathrm{Ly} \alpha)$ correlations reported in the previous section may be due to the primary correlation being with the local continuum for this line (Table 14). Thus the EW is determined by only two parameters, the line flux and the local continuum, whereas for lines whose primary correlation is not with the local continuum, nonsimultaneity and calibration uncertainties between the different continuum regions can induce additional scatter.

\subsubsection{Line Flux versus Driving Continuum Correlations}

We investigate the correlations between the strengths of the broad emission lines such as Ly $\alpha, \mathrm{O}$ VI, C IV, C III], Balmer lines, Fe II, and their driving continua. To determine the driving continua, the SEDs were linearly interpolated between observational points in the OUV. In the X-rays the observed $\alpha_{\mathrm{x}}$ was used, and the EUV continuum was determined by a linear interpolation between the lowest energy point in the X-ray range and the highest in the UV. Then the spectrum was integrated over the energy range of the driving (ionizing) continuum of each line (see Table 14) following the definitions of Krolik \& Kallman (1988; see their Table 4). The probability of a correlation between each line and its driving continuum, as given by the Kendall and Spearman rank tests, is shown in Table 14. The slopes of the linear regression and the scatter of the observational points around the regression line are shown in Table 16. All correlations between the lines and driving continuum are shown in Figure 9. While these correlations are strong, surprisingly in no case were they the primary line versus continuum correlations. In addition, the slopes of the regression lines were always smaller than the expected value of 1 . Possible explanations include differing line and continuum reddening, optically thin (matter bounded) clouds, and continuum emission from an accretion disk (see the discussion in $\S 4.1 .3$ ).

\subsection{Line FWHM versus Continuum Luminosity Correlations}

Only two significant correlations were found between optical/UV line widths and continuum luminosity param- 
PKS1217+023

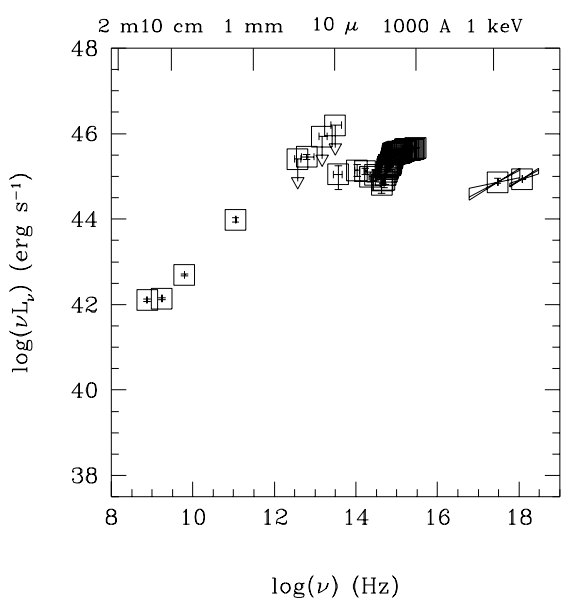

PG2304+042 (PB 5250)

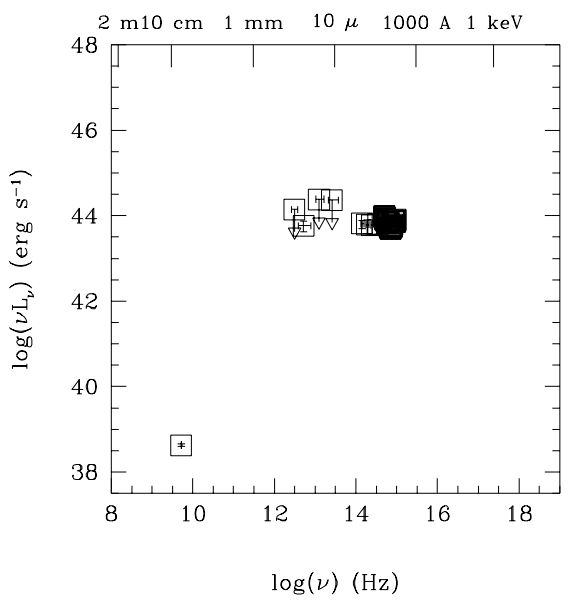

PG $1351+640$

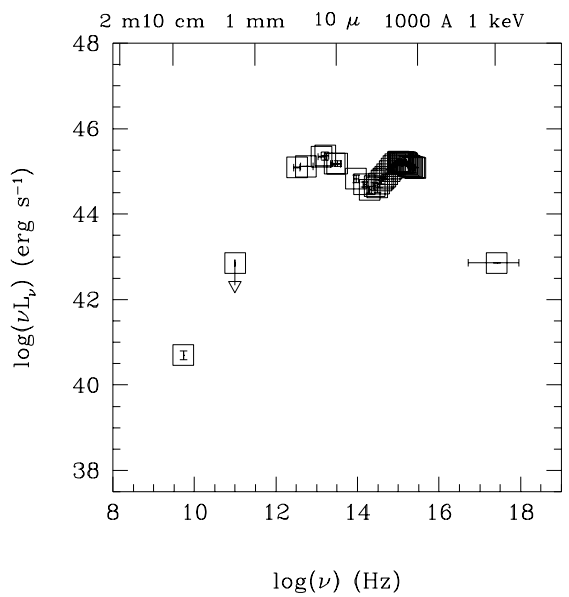

FIG. 3.-Continued

eters: FWHM(NeHe) with far-IR $(10-100 \mu \mathrm{m})$ luminosity $\left(P_{K}=1.9 \%, P_{S}=1.5 \%\right)$ and FWHM ([O III]) with $L_{\text {Ion }}$ $(1.3 \%, 1.3 \%)$. NeHe also correlates but less significantly with $L(0.1-0.2 \mu \mathrm{m})(2.4,2.7 \%)$ and $L_{\text {bol }}(2.2 \%, 1.7 \%)$.

As has been noted in Table 15, the number of spurious correlations with a probability of a chance correlation less than $2 \%$ is expected to be six for the 315 correlations tested. Since we found fewer than six significant correlations, we consider it likely that they are spurious.

\subsection{Correlations vetween Emission-Line Parameters}

We have also studied correlations between the various parameters of each emission line. We shall present here only those correlations that are significant and/or interesting.

The EWs of the Balmer lines correlate with each other, and the $\operatorname{FWHM}(\mathrm{H} \beta)$ correlates with $\operatorname{FWHM}(\mathrm{H} \gamma)$. This is consistent with photoionization models, as the Balmer lines are expected to form in the same region.

The Ly $\alpha$ and C IV line EW and FWHM also correlate with each other (Fig. 10). These correlations are easily understood in the light of standard photoionization models, as it is generally believed that these lines originate in clouds located at the same distance from the continuum source.
However, the presence of a correlation, also reported by Corbin (1991) and Corbin \& Boroson (1996), between the $\mathrm{EW}(\mathrm{Ly} \alpha), \mathrm{EW}(\mathrm{C} \mathrm{IV})$, and $\mathrm{EW}(\mathrm{H} \beta)$, as well as $\mathrm{FWHM}(\mathrm{Ly} \alpha)$, FWHM(C IV), and FWHM(H $\beta)$ (and also FWHM of $\mathrm{H} \gamma$ ), is interesting. $\mathrm{H} \beta$ (and $\mathrm{H} \gamma$ ) is a low-ionization line and is expected to form in a different region than the $\mathrm{C}$ IV and $\mathrm{Ly} \alpha$ lines. If both the high- and low-ionization components scale similarly with luminosity, two models are applicable. First, the Collin-Souffrin et al. (1988) model, where low-ionization lines (LILs) are formed in the atmosphere of the accretion disk, while high-ionization lines (HILs) form in a distinct spherical component. Second, the standard model, where LILs and HILs are formed in the same cloud but in zones of differing ionization.

We see no correlations between the EW and FWHM of individual lines in our sample. An anticorrelation has been reported for C iv in some samples (e.g., Francis et al. 1992; Wills et al. 1993; Corbin \& Francis 1994).

The $\mathrm{EW}([\mathrm{O} \mathrm{III}])$ anticorrelates with $\mathrm{EW}(\mathrm{Fe}$ II) (see Fig. 11). This relation was noted by Boroson \& Green (1992) and dominates their first eigenvector. They rule out an interpretation based on the standard beaming model partly because of the strong dependence on [O III], which is 
TABLE 9

CONTINUUM PARAMETERS

\begin{tabular}{|c|c|c|c|c|c|c|c|}
\hline Name & $\alpha_{o x}$ & $\alpha_{\mathrm{x}}$ & $C_{\mathrm{UV} / \mathrm{IR}}$ & Radio Loud/Quiet & $L_{\mathrm{opt}}{ }^{\mathrm{a}}$ & $L_{\mathrm{x}}$ & $\alpha_{\text {ouv }}{ }^{b}$ \\
\hline III Zw 2 . & 1.14 & 0.4 & 0.47 & $\mathrm{~L}$ & 29.74 & 26.59 & $-0.30 \pm 0.02$ \\
\hline PG $0026+129 \ldots \ldots \ldots$ & 1.41 & 0.9 & 0.49 & Q & 30.58 & 26.92 & $-0.26 \pm 0.03$ \\
\hline $0044+030 \ldots \ldots \ldots \ldots$ & 1.59 & $\ldots$ & $\ldots$ & $\mathrm{L}$ & 31.49 & 27.34 & $\ldots$ \\
\hline I Zw $1 \ldots \ldots \ldots \ldots \ldots$ & 1.42 & 1.7 & $\ldots$ & Q & 30.07 & 26.36 & $\ldots$ \\
\hline PHL $909 \ldots \ldots \ldots \ldots$ & 1.28 & 0.3 & -0.08 & Q & 30.09 & 26.27 & $\ldots$ \\
\hline 3С $47 \ldots \ldots \ldots \ldots \ldots$ & 1.01 & 0.9 & 0.00 & $\mathrm{~L}$ & 30.34 & 27.71 & $\ldots$ \\
\hline $3 \mathrm{C} 48 \ldots \ldots \ldots \ldots \ldots \ldots$ & 1.28 & 0.7 & 0.18 & $\mathrm{~L}$ & 30.77 & 27.45 & $-0.61 \pm 0.16$ \\
\hline NAB $0205+024 \ldots \ldots$ & 1.33 & 1.2 & 0.56 & $\mathrm{~L}$ & 30.33 & 26.87 & $-0.43 \pm 0.06$ \\
\hline PKS $0312-770 \ldots \ldots$ & 1.32 & 0.1 & $\ldots$ & $\mathrm{L}$ & 30.54 & 27.08 & $\ldots$ \\
\hline PKS $0637-752 \ldots \ldots$ & 1.29 & 0.5 & $\ldots$ & $\mathrm{L}$ & 31.67 & 28.35 & $\ldots$ \\
\hline PG $0804+760 \ldots \ldots \ldots$ & 1.28 & 0.0 & 0.60 & Q & 30.14 & 26.80 & $-0.36 \pm 0.08$ \\
\hline 3C $206 \ldots \ldots \ldots \ldots \ldots$ & 1.24 & 0.7 & 0.49 & $\mathrm{~L}$ & 30.59 & 27.36 & $0.51 \pm 0.03$ \\
\hline PG $0844+349 \ldots \ldots \ldots$ & 1.65 & 0.6 & 0.44 & Q & 30.21 & 25.93 & $-0.61 \pm 0.04$ \\
\hline 3C $215 \ldots \ldots \ldots \ldots \ldots$ & 1.14 & 0.0 & 0.00 & $\mathrm{~L}$ & 30.11 & 27.16 & $\ldots$ \\
\hline Mrk $704 \ldots \ldots \ldots \ldots$ & 1.35 & 0.3 & $\ldots$ & Q & 29.15 & 25.63 & $\ldots$ \\
\hline $0923+392 \ldots \ldots \ldots \ldots$ & 1.12 & 0.4 & 0.64 & $\mathrm{~L}$ & 30.82 & 27.97 & $\ldots$ \\
\hline PG $1001+054 \ldots \ldots \ldots$ & $>1.76$ & $\ldots$ & $\ldots$ & Q & 30.16 & $<25.57$ & $\ldots$ \\
\hline PG $1004+130 \ldots \ldots \ldots$ & $>1.84$ & $\ldots$ & $\ldots$ & Q & 30.64 & $<25.86$ & $\ldots$ \\
\hline PG $1012+008 \ldots \ldots \ldots$ & $\ldots$ & $\ldots$ & 0.25 & Q & 30.36 & $\ldots$ & \\
\hline B21028 + $103 \ldots \ldots \ldots$ & 1.11 & 0.5 & 0.46 & $\mathrm{~L}$ & 29.87 & 27.00 & $-0.26 \pm 0.04$ \\
\hline 3C $249.1 \ldots \ldots \ldots \ldots$ & 1.33 & 1.0 & 0.30 & $\mathrm{~L}$ & 30.89 & 27.45 & $-0.45 \pm 0.02$ \\
\hline PG $1116+215 \ldots \ldots \ldots$ & 1.39 & 1.0 & 0.59 & $\mathrm{Q}$ & 30.63 & 27.02 & $-0.24 \pm 0.05$ \\
\hline PG $1121+422 \ldots \ldots \ldots$ & $\ldots$ & $\ldots$ & $\ldots$ & Q & 30.26 & $\ldots$ & \\
\hline 3C $263 \ldots \ldots \ldots \ldots \ldots$ & 1.31 & 0.7 & 0.70 & $\mathrm{~L}$ & 31.31 & 27.91 & $\ldots$ \\
\hline GQ Comae ........... & 1.38 & 1.1 & 0.25 & Q & 30.63 & 27.04 & $-0.70 \pm 0.07$ \\
\hline PG $1211+143 \ldots \ldots \ldots$ & 1.17 & 1.8 & 0.46 & Q & 30.19 & 27.16 & $-0.87 \pm 0.04$ \\
\hline PKS $1217+023 \ldots \ldots$ & 1.16 & 0.5 & 0.48 & $\mathrm{~L}$ & 30.38 & 27.32 & $\ldots$ \\
\hline 3C $273 \ldots \ldots \ldots \ldots \ldots$ & 1.30 & 0.3 & 0.32 & $\mathrm{~L}$ & 31.46 & 28.07 & $\ldots$ \\
\hline PG $1307+085 \ldots \ldots \ldots$ & 1.43 & 0.9 & 0.61 & Q & 30.47 & 26.97 & $-0.40 \pm 0.04$ \\
\hline PG $1351+640 \ldots \ldots \ldots$ & $>1.76$ & $\ldots$ & $\ldots$ & Q & 29.91 & $<25.32$ & $\ldots$ \\
\hline PG $1407+265 \ldots \ldots \ldots$ & 1.44 & 1.2 & 0.64 & Q & 32.01 & 28.47 & $\ldots$ \\
\hline PG $1416-129 \ldots \ldots \ldots$ & 1.26 & 0.9 & 0.53 & Q & 30.39 & 26.93 & $-0.07 \pm 0.05$ \\
\hline PG $1426+015 \ldots \ldots \ldots$ & 1.36 & 0.9 & 0.69 & Q & 30.04 & 26.70 & $0.09 \pm 0.03$ \\
\hline Mrk $841 \ldots \ldots \ldots \ldots$ & 1.30 & 1.0 & 0.38 & $\mathrm{Q}$ & 29.24 & 26.32 & $\ldots$ \\
\hline PG $1613+658 \ldots \ldots \ldots$ & 1.32 & 1.1 & 0.28 & Q & 30.27 & 26.85 & $-0.28 \pm 0.05$ \\
\hline 3C $351 \ldots \ldots \ldots \ldots \ldots$ & 1.60 & 0.1 & 0.40 & $\mathrm{~L}$ & 31.02 & 26.86 & $\ldots$ \\
\hline $1721+343 \ldots \ldots \ldots \ldots$ & 0.98 & 0.5 & 0.72 & $\mathrm{~L}$ & 30.09 & 27.54 & $-0.10 \pm 0.04$ \\
\hline KAZ $102 \ldots \ldots \ldots \ldots$ & 1.41 & -0.2 & 0.40 & Q & 30.11 & 26.44 & $-0.46 \pm 0.04$ \\
\hline II Zw $136 \ldots \ldots \ldots \ldots$ & 1.46 & 0.8 & 0.42 & $\mathrm{Q}$ & 29.96 & 26.32 & $-0.49 \pm 0.06$ \\
\hline PHL 1657 ........... & 1.32 & 0.5 & 0.15 & $\mathrm{~L}$ & 30.62 & 27.19 & $-0.78 \pm 0.04$ \\
\hline PG $2304+042 \ldots \ldots \ldots$ & $\ldots$ & $\ldots$ & $\ldots$ & Q & $\ldots$ & $\ldots$ & \\
\hline
\end{tabular}

${ }^{\text {a }} L_{\text {opt }}$ is a standard definition of luminosity at $2500 \AA$ obtained by extrapolating $B$ (or $V$ ) magnitude and assuming a continuum slope of 0.5 .

b Slope $\left(f_{v} \propto v^{-\alpha}\right)$ between 1285 and $5100 \AA ̊$, taken from Kuhn 1996.

thought to be isotropic and partly because of the positive correlation of absolute continuum magnitude with the magnitude of [O III], which is not expected if beaming causes the range in optical continuum flux. Instead, they favor a model based on a geometric effect intrinsic to the quasar, such as shadowing of the narrow-line region (NLR) by a coplanar, toroidal-shaped broad-line region (BLR) so that the covering factor, perhaps determined by the ratio of accretion rate to the Eddington rate, drives the correlations. Eddington-limited flow was also suggested to explain those objects with extremely strong Fe II emission (Pounds et al. 1996) based primarily on their unusually steep, soft X-ray spectra.

Since $[\mathrm{O} \mathrm{III}]$ and $[\mathrm{O} \mathrm{II}]$ originate in similar parts of the NLR, we expect their emission-line profiles to be similar. We find a correlation between their EWs, but we do not see a correlation for their FWHM. However, we do not place much weight on this latter result, as the narrow lines are often close to the spectral resolution, [O II] is weak, and we have only 19 measurements of variable quality.

\subsection{Continuum-Continuum Correlations}

All the continuum luminosities correlate with one another. The regression slopes for $L_{1}$ versus $L_{2}$ and $L_{2}$ versus $L_{1}$ relations are presented in Table 17. Most of the relations have regression slopes near unity. The exception is the $L(0.1-0.2 \mu \mathrm{m})$ versus $L(0.2-0.4 \mu \mathrm{m})$ relation, whose slope is less than 1 , indicating that $L(0.1-0.2 \mu \mathrm{m})$ increases faster with increasing bolometric luminosity than any other luminosity. This could be explained if the peak of the big blue bump (BBB) moves from the EUV into the UV as the luminosity increases.

Correlations with the spectral indices were also investigated. The only correlations found were those between $L_{\mathrm{x}}$, $L(1-2 \mathrm{keV})$, and $\alpha_{\mathrm{ox}}$ (regression slope $=-1.51 \pm 0.83$ and $-1.88 \pm 0.7$, respectively), showing the steepening of $\alpha_{o x}$ 
TABLE 10

DeCADE LUMINOSITIES ${ }^{\mathrm{a}}$

\begin{tabular}{|c|c|c|c|c|c|}
\hline \multirow[b]{2}{*}{ NAME } & \multicolumn{5}{|c|}{ LUMINOSITY } \\
\hline & $10-100 \mu \mathrm{m}$ & $1-10 \mu \mathrm{m}$ & $0.1-1 \mu \mathrm{m}$ & $0.1-1 \mathrm{keV}$ & $1-10 \mathrm{keV}$ \\
\hline $0007+106 \ldots \ldots$ & 45.110 & 45.240 & 45.410 & 44.300 & 44.970 \\
\hline $0026+129 \ldots \ldots$ & $<45.020$ & 45.390 & 45.720 & 44.790 & 44.920 \\
\hline $0044+030 \ldots \ldots$ & $<46.910$ & 45.880 & 46.110 & $\ldots$ & $\ldots$ \\
\hline $0050+124 \ldots \ldots$ & 45.670 & 45.540 & 45.270 & 45.070 & $\ldots$ \\
\hline $0054+145 \ldots \ldots$ & 45.660 & 45.630 & 45.590 & 44.240 & 44.820 \\
\hline $0133+207 \ldots \ldots$ & 46.240 & 45.930 & 45.930 & 45.550 & $\ldots$ \\
\hline $0134+329 \ldots \ldots$ & 46.610 & 46.040 & 46.020 & 45.090 & $\ldots$ \\
\hline $0205+024 \ldots \ldots$ & 45.350 & 45.370 & 45.680 & 44.550 & $\ldots$ \\
\hline $0312-770 \ldots \ldots$ & $<45.560$ & 45.210 & 45.910 & 44.350 & $\ldots$ \\
\hline $0637-752 \ldots \ldots$ & 46.770 & 46.820 & 47.030 & 45.880 & 46.240 \\
\hline $0804+761 \ldots \ldots$ & 45.330 & 45.530 & 45.700 & 44.710 & 44.690 \\
\hline $0837-120 \ldots \ldots$ & 45.200 & 45.400 & 45.640 & 45.060 & 45.220 \\
\hline $0844+349 \ldots \ldots$ & 44.800 & 44.730 & 45.180 & 43.470 & $\ldots$ \\
\hline $0903+169 \ldots \ldots$ & $<46.490$ & $<46.430$ & 45.550 & 44.680 & $\ldots$ \\
\hline $0915+165 \ldots \ldots$ & 44.620 & 44.640 & 44.160 & 43.120 & $\ldots$ \\
\hline $0923+392 \ldots \ldots$ & $<46.170$ & $<46.540$ & 46.540 & 45.340 & 45.750 \\
\hline $1001+054 \ldots \ldots$ & 44.960 & 45.340 & 45.330 & $\ldots$ & $\ldots$ \\
\hline $1004+130 \ldots \ldots$ & 45.860 & 45.780 & 46.200 & $\ldots$ & $\ldots$ \\
\hline $1012+008 \ldots \ldots$ & $<45.690$ & 45.290 & 45.560 & $\ldots$ & $\ldots$ \\
\hline $1028+313 \ldots \ldots$ & $<45.640$ & 45.160 & 45.520 & 44.520 & 44.970 \\
\hline $1100+772 \ldots \ldots$ & 45.590 & 45.870 & 46.220 & 45.260 & $\ldots$ \\
\hline $1116+215 \ldots \ldots$ & $<45.470$ & 45.890 & 46.250 & 44.740 & $\ldots$ \\
\hline $1121+422 \ldots \ldots$ & $<45.730$ & 45.480 & 45.580 & $\ldots$ & $\ldots$ \\
\hline $1137+661 \ldots \ldots$ & $<46.460$ & 46.120 & 46.730 & 45.730 & 45.880 \\
\hline $1202+281 \ldots \ldots$ & 45.560 & 45.420 & 45.380 & 44.980 & $\ldots$ \\
\hline $1211+143 \ldots \ldots$ & 45.410 & 45.410 & 45.640 & 45.620 & 44.710 \\
\hline $1217+023 \ldots \ldots$ & 45.670 & 45.450 & 46.000 & 44.960 & 45.300 \\
\hline $1226+023 \ldots \ldots$ & 46.440 & 46.480 & 46.790 & 45.480 & 46.020 \\
\hline $1307+085 \ldots \ldots$ & 45.320 & 45.290 & 45.780 & 44.640 & 44.920 \\
\hline $1351+640 \ldots \ldots$ & 45.590 & 45.250 & 45.430 & $\ldots$ & $\ldots$ \\
\hline $1407+265 \ldots \ldots$ & 46.780 & 46.840 & 47.040 & 46.450 & 46.190 \\
\hline $1416-129 \ldots \ldots$ & $<45.420$ & 44.750 & 45.410 & 44.900 & 44.880 \\
\hline $1426+015 \ldots \ldots$ & 45.190 & 45.180 & 45.640 & 44.830 & 44.700 \\
\hline $1501+106 \ldots \ldots$ & 44.680 & 44.540 & 44.720 & 44.030 & 44.150 \\
\hline $1613+658 \ldots \ldots$ & 45.690 & 45.420 & 45.530 & 44.830 & $\ldots$ \\
\hline $1704+608 \ldots \ldots$ & 46.270 & 46.240 & 46.310 & 44.410 & $\ldots$ \\
\hline $1721+343 \ldots \ldots$ & 45.700 & 45.560 & 46.070 & 45.060 & 45.470 \\
\hline $1803+676 \ldots \ldots$ & $<44.940$ & 45.280 & 45.520 & 43.650 & $\ldots$ \\
\hline $2130+099 \ldots \ldots$ & 45.100 & 45.090 & 45.160 & 43.750 & 44.040 \\
\hline $2135-147 \ldots \ldots$ & 45.660 & 45.570 & 45.780 & 44.940 & 45.340 \\
\hline $2304+042 \ldots \ldots$ & 44.100 & 44.160 & $\ldots$ & $\ldots$ & $\ldots$ \\
\hline
\end{tabular}

${ }^{\text {a }}$ The luminosities in the table are $\log v L_{v}$, in ergs $\mathrm{s}^{-1}$.

with decreasing X-ray luminosity and the correlation between $L_{\mathrm{x}}$ and $L_{\mathrm{opt}}$ (regression slope $=0.74 \pm 0.15$, which is in agreement with the Wilkes et al. 1994 slope of 0.71). We do not find the correlation between $\alpha_{\text {ouv }}$ and $\alpha_{\mathrm{x}}$ reported by Puchnarewicz et al. $(1996)$ or that of $\alpha_{o x}$ with $\alpha_{x}$ also reported by Laor et al. (1997; see $\S 4.1 .5$ ).

The mean SEDs for high- and low-luminosity quasars in our sample are shown separately in Figure 12. The quasars that contribute to the high-luminosity mean are $0637-752$, $1226+023,1407+265$, and $1704+608[L(0.8-1.6 \mu \mathrm{m})>$ 45.5], and those that contributed to the low-luminosity mean are: $0007+106,0026+129,0804+761,1416-129$, $1501+106[L(0.8-1.6 \mu \mathrm{m})<44.2]$, and $\mathrm{EW}(\mathrm{H} \beta)>70 \AA$. The low-luminosity object $1001+054$ was omitted while making the mean, as we had no information on the X-ray slope, and the large errors in starlight caused large uncertainties in the $\sim 1 \mu \mathrm{m}$ region. The higher luminosity SEDs show less dispersion around the median in the IR-UV bands than the lower luminosity SEDs. The range in X-rays is systematically shifted to lower X-rays for the higher luminosity SEDs, and the BBB is stronger (in the UV) for lower luminosity SEDs (meaning, as was also noticed from the slope analysis, that the peak of the BBB moves from the EUV into the UV as the luminosity increases). However, the range in the SEDs present is broad and overlaps significantly between the two samples.

\section{DISCUSSION}

\subsection{The Baldwin Effect}

In a simple, radiation-bounded photoionization model, one would expect the line flux in the HILs such as C IV and $\mathrm{Ly} \alpha$ to be proportional to the strength of the ionizing continuum as a larger fraction of emitting material reaches the ionization state of the line. Since the EW is the ratio of line flux to that in the local continuum (i.e., in the same wave- 
TABLE 11

OCTAVE LUMINOSITIES $^{\text {a }}$

\begin{tabular}{|c|c|c|c|c|c|c|}
\hline \multirow[b]{2}{*}{ NAME } & \multicolumn{6}{|c|}{ LUMINOSITY } \\
\hline & $0.8-1.6 \mu \mathrm{m}$ & $0.4-0.8 \mu \mathrm{m}$ & $0.2-0.4 \mu \mathrm{m}$ & $0.1-0.2 \mu \mathrm{m}$ & $0.15-0.3 \mathrm{keV}$ & $1-2 \mathrm{keV}$ \\
\hline $0007+106 \ldots \ldots$ & 44.470 & 44.640 & 44.970 & 45.020 & 43.650 & 44.140 \\
\hline $0026+129 \ldots \ldots$ & 44.870 & 45.010 & 45.230 & 45.340 & 44.250 & 44.350 \\
\hline $0044+030 \ldots \ldots$ & 45.580 & 45.470 & 45.640 & 45.640 & $\ldots$ & $\ldots$ \\
\hline $0050+124 \ldots \ldots$ & 44.770 & 44.750 & 44.830 & 44.650 & 44.630 & 43.890 \\
\hline $0054+145 \ldots \ldots$ & 44.880 & 45.010 & 45.150 & 45.070 & 43.570 & 44.120 \\
\hline $0133+207 \ldots \ldots$ & 45.090 & 45.030 & 45.420 & 45.650 & 45.030 & 45.030 \\
\hline $0134+329 \ldots \ldots$ & 45.020 & 45.260 & 45.580 & 45.660 & 44.510 & 44.760 \\
\hline $0205+024 \ldots \ldots$ & 44.570 & 44.870 & 45.260 & 45.320 & 44.110 & 44.010 \\
\hline $0312-770 \ldots \ldots$ & 44.760 & 45.120 & 45.260 & 45.680 & 43.610 & 44.350 \\
\hline $0637-752 \ldots \ldots$ & 46.230 & 46.330 & 46.460 & 46.720 & $<44.960$ & 45.540 \\
\hline $0804+761 \ldots \ldots$ & 44.700 & 44.880 & 45.310 & 45.310 & 44.190 & 44.180 \\
\hline $0837-120 \ldots \ldots$ & 44.630 & 44.690 & 45.070 & 45.400 & 44.470 & 44.760 \\
\hline $0844+349 \ldots \ldots$ & 44.100 & 44.470 & 44.770 & 44.760 & 42.850 & 43.230 \\
\hline $0903+169 \ldots \ldots$ & $<45.480$ & 44.980 & 44.910 & 45.180 & 44.050 & 44.470 \\
\hline $0915+165 \ldots \ldots$ & 43.610 & 43.590 & 43.750 & 43.570 & 42.450 & 42.990 \\
\hline $0923+392 \ldots \ldots$ & $<46.030$ & $<46.100$ & 45.950 & 46.110 & 44.680 & 45.180 \\
\hline $1001+054 \ldots \ldots$ & 44.790 & 44.330 & 45.000 & 44.870 & $\ldots$ & 0.000 \\
\hline $1004+130 \ldots \ldots$ & 45.190 & 45.470 & 45.860 & 45.730 & $\ldots$ & 0.000 \\
\hline $1012+008 \ldots \ldots$ & 44.780 & 44.800 & 44.970 & 45.260 & $\ldots$ & 0.000 \\
\hline $1028+313 \ldots \ldots$ & 44.590 & 44.730 & 44.960 & 45.250 & 43.930 & 44.300 \\
\hline $1100+772 \ldots \ldots$ & 45.270 & 45.460 & 45.730 & 45.870 & 44.740 & 44.740 \\
\hline $1116+215 \ldots \ldots$ & 45.200 & 45.390 & 45.790 & 45.950 & 44.220 & 44.220 \\
\hline $1121+422 \ldots \ldots$ & 44.810 & 45.010 & 45.160 & $\ldots$ & $\ldots$ & $\ldots$ \\
\hline $1137+661 \ldots \ldots$ & 45.630 & 45.940 & 46.210 & 46.430 & 45.150 & 45.400 \\
\hline $1202+281 \ldots \ldots$ & 44.410 & 44.500 & 44.940 & 45.050 & 44.470 & 44.390 \\
\hline $1211+143 \ldots \ldots$ & 44.760 & 44.960 & 45.220 & 45.200 & 45.190 & 44.370 \\
\hline $1217+023 \ldots \ldots$ & 44.890 & 45.280 & 45.680 & 45.480 & 44.330 & 44.740 \\
\hline $1226+023 \ldots \ldots$ & 45.870 & 46.030 & 46.320 & 46.440 & 44.850 & 45.330 \\
\hline $1307+085 \ldots \ldots$ & 44.740 & 45.010 & 45.300 & 45.450 & 44.100 & 44.190 \\
\hline $1351+640 \ldots \ldots$ & 44.490 & 44.790 & 45.040 & 44.960 & $\ldots$ & $\ldots$ \\
\hline $1407+265 \ldots \ldots$ & 46.040 & 46.240 & 46.570 & 46.720 & 45.960 & 45.800 \\
\hline $1416-129 \ldots \ldots$ & 44.210 & 44.450 & 45.020 & 45.060 & 44.360 & 44.450 \\
\hline $1426+015 \ldots \ldots$ & 44.530 & 44.660 & 45.120 & 45.390 & 44.300 & 44.200 \\
\hline $1501+106 \ldots \ldots$ & 43.680 & 43.820 & 44.290 & 44.410 & 43.540 & 43.610 \\
\hline $1613+658 \ldots \ldots$ & 44.560 & 44.750 & 45.090 & 45.180 & 44.310 & 44.220 \\
\hline $1704+608 \ldots \ldots$ & 45.510 & 45.640 & 45.810 & 45.940 & 43.750 & 44.250 \\
\hline $1721+343 \ldots \ldots$ & 44.940 & 45.210 & 45.600 & 45.750 & 44.430 & 44.850 \\
\hline $1803+676 \ldots \ldots$ & 44.640 & 44.760 & 45.060 & 45.150 & 42.810 & 43.800 \\
\hline $2130+099 \ldots \ldots$ & 44.120 & 44.340 & 44.740 & 44.810 & 43.240 & 43.420 \\
\hline $2135-147 \ldots \ldots$ & 44.860 & 45.200 & 45.380 & 45.220 & 44.340 & 44.560 \\
\hline $2304+042 \ldots \ldots$ & 43.640 & 43.990 & $\ldots$ & $\ldots$ & $\ldots$ & $\ldots$ \\
\hline
\end{tabular}

${ }^{\text {a }}$ The luminosities in the table are $\log v L_{v}, \mathrm{ergs} \mathrm{s}^{-1}$.

length region as the line), the Baldwin effect indicates that the line flux is increasing more slowly than the local continuum (or is constant). One possible interpretation is that the ionizing/heating continuum for the emission line (i.e., its driving continuum) is increasing more slowly than the local continuum, suggesting that the continuum shape correlates with the luminosity of a quasar. A number of papers have reported such a correlation. Tananbaum et al. (1986), Wilkes et al. (1994), and Green et al. (1995) note that the power law between UV and soft X-rays, $\alpha_{\mathrm{ox}}$, increases significantly with luminosity. This results in the soft X-ray luminosity being weaker relative to the UV in higher luminosity quasars.

\subsubsection{Zheng \& Malkan Model}

Zheng \& Malkan (1993), studying optical and UV properties of quasars and Seyfert galaxies, interpreted their color-luminosity relations as the result of a shift of the BBB toward lower energies in higher OUV luminosity objects. In this scenario higher luminosity quasars are predicted to have a lower fraction of higher energy ionizing photons available relative to the optical/UV continuum. This change in continuum shape would cause the EWs of highionization emission lines sensitive to the $\mathrm{X}$-ray continuum, such as $\mathrm{C}$ IV and $\mathrm{He}$ II, to decrease at high luminosities relative to lower ionization emission lines, such as $\mathrm{Ly} \alpha$, $\mathrm{C}$ III], and the Balmer lines. The scenario predicts a stronger and more easily detectable Baldwin effect for higher ionization lines. We do see a shift in the SEDs, but our data do not favor this model, as we see the Baldwin effect in both HILs and LILs.

\subsubsection{Mushotzky \& Ferland Model}

Mushotzky \& Ferland (1984) explain the Baldwin effect as due to a systematic decrease in ionization parameter, $U$, as the luminosity increases. As the ionization parameter 
TABLE 12

BROADBAND LUMINOSITIES

\begin{tabular}{|c|c|c|c|c|}
\hline Name & $L_{\text {UVOIR }}{ }^{\mathrm{a}}$ & $L_{\mathrm{bol}}{ }^{\mathbf{b}}$ & Lyman Continuum $^{c}$ & $L_{\text {Ion }}{ }^{\mathrm{d}}$ \\
\hline $0007+106 \ldots$ & 45.750 & 45.920 & 45.320 & 44.730 \\
\hline $0026+129 \ldots \ldots$ & 45.920 & 46.120 & 45.640 & 45.200 \\
\hline $0044+030 \ldots \ldots$ & 46.340 & 46.370 & $\ldots$ & $\ldots$ \\
\hline $0050+124 \ldots \ldots$ & 46.000 & 46.100 & 45.320 & 44.690 \\
\hline $0054+145 \ldots \ldots$ & 46.100 & 46.220 & 45.490 & 45.000 \\
\hline $0133+207 \ldots \ldots$ & 46.540 & 46.750 & 46.170 & 45.630 \\
\hline $0134+329 \ldots \ldots$ & 46.790 & 46.920 & 45.930 & 45.490 \\
\hline $0205+024 \ldots \ldots$ & 45.970 & 46.170 & 45.720 & 45.290 \\
\hline $0312-770 \ldots \ldots$ & 46.010 & 46.240 & 45.780 & 45.510 \\
\hline $0637-752 \ldots \ldots$ & 47.370 & 47.550 & 46.920 & 46.560 \\
\hline $0804+761 \ldots \ldots$ & 46.020 & 46.160 & 45.550 & 45.150 \\
\hline $0837-120 \ldots \ldots$ & 45.920 & 46.220 & 45.870 & 45.390 \\
\hline $0844+349 \ldots \ldots$ & 45.430 & 45.490 & 44.610 & 44.320 \\
\hline $0903+169 \ldots \ldots$ & 46.020 & 45.790 & 45.550 & 45.060 \\
\hline $0915+165 \ldots \ldots$ & 45.000 & 45.050 & 43.890 & 43.370 \\
\hline $0923+392 \ldots \ldots$ & 46.810 & 47.100 & 46.390 & 45.940 \\
\hline $1001+054 \ldots \ldots$ & 45.720 & 45.780 & 44.880 & 44.570 \\
\hline $1004+130 \ldots \ldots$ & 46.460 & 46.550 & 45.690 & 45.430 \\
\hline $1012+008 \ldots \ldots$ & 45.760 & 45.770 & $\ldots$ & $\ldots$ \\
\hline $1028+313 \ldots \ldots$ & 45.730 & 46.040 & 45.720 & 45.270 \\
\hline $1100+772 \ldots \ldots$ & 46.440 & 46.630 & 46.150 & 45.780 \\
\hline $1116+215 \ldots \ldots$ & 46.440 & 46.580 & 45.980 & 45.720 \\
\hline $1121+422 \ldots \ldots$ & 46.020 & $\ldots$ & $\ldots$ & $\ldots$ \\
\hline $1137+661 \ldots \ldots$ & 46.850 & 47.110 & 46.720 & 46.320 \\
\hline $1202+281 \ldots \ldots$ & 45.940 & 46.110 & 45.550 & 45.020 \\
\hline $1211+143 \ldots \ldots$ & 45.980 & 46.260 & 45.920 & 45.330 \\
\hline $1217+023 \ldots \ldots$ & 46.240 & 46.430 & 45.850 & 45.360 \\
\hline $1226+023 \ldots \ldots$ & 47.080 & 47.280 & 46.690 & 46.280 \\
\hline $1307+085 \ldots \ldots$ & 46.000 & 46.160 & 45.660 & 45.270 \\
\hline $1351+640 \ldots \ldots$ & 45.920 & 46.000 & 44.920 & 44.650 \\
\hline $1407+265 \ldots \ldots$ & 47.380 & 47.640 & 47.270 & 46.830 \\
\hline $1416-129 \ldots \ldots$ & 45.500 & 45.810 & 45.500 & 44.910 \\
\hline $1426+015 \ldots \ldots$ & 45.870 & 46.090 & 45.640 & $<45.530$ \\
\hline $1501+106 \ldots \ldots$ & 45.130 & 45.350 & 44.930 & 44.420 \\
\hline $1613+658 \ldots \ldots$ & 46.040 & 46.170 & 45.480 & $<45.190$ \\
\hline $1704+608 \ldots \ldots$ & 46.750 & 46.820 & 45.910 & 45.670 \\
\hline $1721+343 \ldots \ldots$ & 46.310 & 46.510 & 46.010 & 45.530 \\
\hline $1803+676 \ldots \ldots$ & 45.870 & 45.740 & 45.050 & 44.760 \\
\hline $2130+099 \ldots \ldots$ & 45.600 & 45.730 & 45.110 & 44.720 \\
\hline $2135-147 \ldots \ldots$ & 46.160 & 46.320 & 45.720 & $<45.280$ \\
\hline $2304+042 \ldots \ldots$ & 44.620 & $\ldots$ & $\cdots$ & $\cdots$ \\
\hline
\end{tabular}

${ }^{\text {a }}$ UV/OIR luminosity between 100 and $0.1 \mu \mathrm{m}$.

b Bolometric luminosity $1 \mathrm{~m}-10 \mathrm{keV}$.

c Lyman continuum $912 \AA-10 \mathrm{keV}$.

${ }^{\mathrm{d}}$ Ionizing photon rate multiplied by 1 Ryd. As $1 \mathrm{Ryd}=R=2.18$ $\times 10^{-11}$ ergs, $N_{\text {Ion }} R=10^{44} N_{44}$ ergs $\mathrm{s}^{-1}$ and $N_{\text {Ion }}=4.6 \times 10^{54} N_{44}$ photons $\mathrm{s}^{-1}$.

depends on the number of ionizing photons, $U$ depends on the continuum shape. Their model assumes a single-zone BLR, i.e., determines the ionization for one "slab" at a given distance from the central source and assumes spherical symmetry. The model predicts the presence of a Baldwin effect for the $\mathrm{C}$ IV line, as the C IV luminosity increases rapidly with increasing $U$ (decreasing UV luminosity and $\left.\alpha_{o x}\right)$ in the range $30-32$ in $L_{1450}\left(\log L_{v}[2450 \AA]\right)$. This relation flattens around $L_{1450}<30$ and so predicts a weaker/or no Baldwin effect for lower luminosity active galactic nuclei (AGNs; see Fig. 1 in Mushotzky \& Ferland 1984). In addition, their calculation showed that there should not exist a Baldwin effect in Ly $\alpha, \mathrm{C}$ III], $\mathrm{H} \alpha$, and $\mathrm{H} \beta$ lines, as the luminosity of these lines decreases with increasing $U$ (i.e., decreasing luminosity) in the same luminosity range. It also follows that the EWs of Ly $\alpha, \mathrm{C}$ III], and C IV should, in this scenario, be relatively independent of both $\alpha_{o x}$ and $\alpha_{x}$, as the lines do not originate in the X-ray heated zones deep in the emission-line clouds.

In our sample this model matches only the $\mathrm{C}$ IV line, for which we see the Baldwin effect for $L_{1450}>29.7$ [ $L(0.1-0.2$ $\mu \mathrm{m})>45]$. The four narrow-line low-luminosity AGNs that have $L(0.1-0.2 \mu \mathrm{m})<45$ destroy the anticorrelation (with these objects the probability of a chance correlation is $P>5 \%$, without $P<1 \%$ ) and fall into the region where the model predicts no Baldwin effect (as it is the region where $\mathrm{C}$ IV remains fairly constant with increasing $U$ ). However, we see a strong correlation for EW(C IV) with $\alpha_{\mathrm{ox}}$, as well as less significant correlations for $\mathrm{Ly} \alpha, \mathrm{H} \beta$, and $\mathrm{O}$ VI with spectral shape, none of which are consistent with the Mushotzky \& Ferland model. Our data also show a Baldwin effect for Ly $\alpha$ and the Balmer lines that the model does not predict. The model also predicts that the $\mathrm{C}$ IV/Ly $\alpha$ ratio should decrease with increasing luminosity, but no such relation is found in our sample. We conclude that this model does not provide a good explanation for the relations present in our sample and so reject a pure photoionization scenario for the BELR.

\subsubsection{The Accretion Disk Model}

Netzer $(1985,1987)$ and Netzer, Laor, \& Gondhalekar (1992), using an optically thick and geometrically thin accretion disk, explain the Baldwin effect in a different way. The model assumes a distribution of identical clouds at distances from the center of the disk greater than the size of the UV-emitting part of the disk. The incident ionizing flux from the accretion disk (AD) is composed of two components: the UV continuum, which is viewing-angle dependent because of limb darkening and change in projected surface area, and the X-ray component, which is viewingangle independent. The UV emission is strongest when the disk is viewed face-on. Because the BELR radiates more isotropically than the UV disk, a random selection of objects differing only in viewing angle would result in measurements of constant line luminosities but varying UV continuum luminosities, producing an anticorrelation of $\mathrm{EW}$ with $L_{\mathrm{Uv}}$. In addition, an anticorrelation between EW and $\alpha_{o x}$ is expected, as objects viewed face-on would have steeper $\alpha_{\text {ox }}$ (due to relatively higher UV-to-X-ray emission) and smaller EW than those seen edge-on. Correlations of the more isotropic X-ray emission with line EW would be much weaker.

The continuum viewed by the BELR clouds themselves is a function of viewing angle. As a result, clouds at small viewing angles produce strong $\mathrm{Ly} \alpha, \mathrm{O} \mathrm{VI}$, and Balmer continuum emission because of the stronger UV ionizing continuum. Clouds at large viewing angles, where the fraction of high-energy X-ray photons is largest, emit low-excitation lines such as Fe II and $\mathrm{Mg}$ II. The model predicts that in clouds at these viewing angles, Fe II emission can reach an intensity comparable with that of $\operatorname{Ly} \alpha$ and more than 10 times that of $\mathrm{H} \beta$. Under our assumption of optically thin emitting clouds, this viewing-angle dependence will not affect the observed line strengths unless external effects, such as viewing-angle-dependent reddening, are also present.

In general the results presented here show good agreement with the predictions of the AD model. We see an anticorrelation of EW with UV luminosity for $\mathrm{Ly} \alpha, \mathrm{H} \beta, \mathrm{H} \delta$, and a less significant relation for $\mathrm{C}$ III], although this 


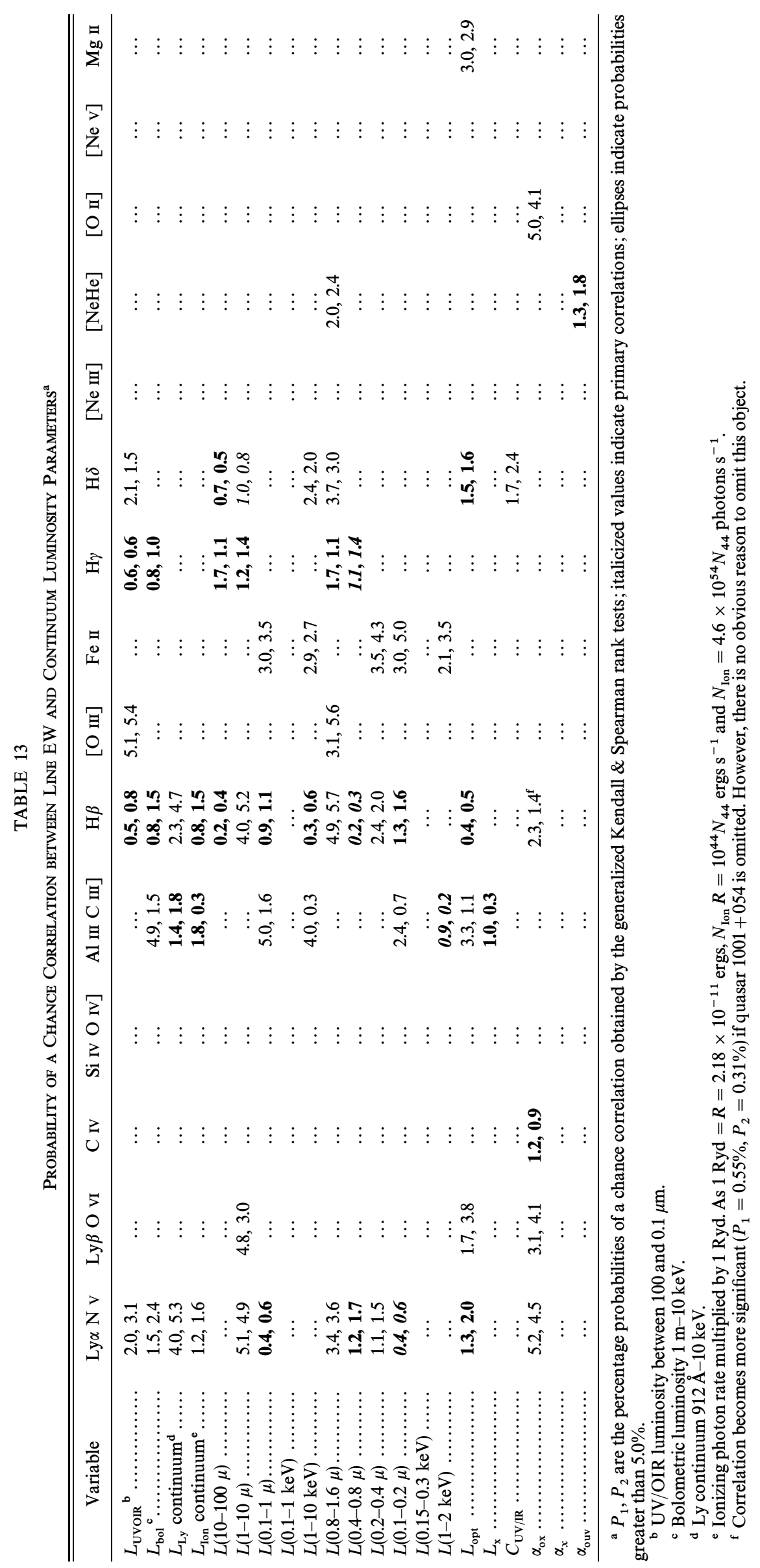




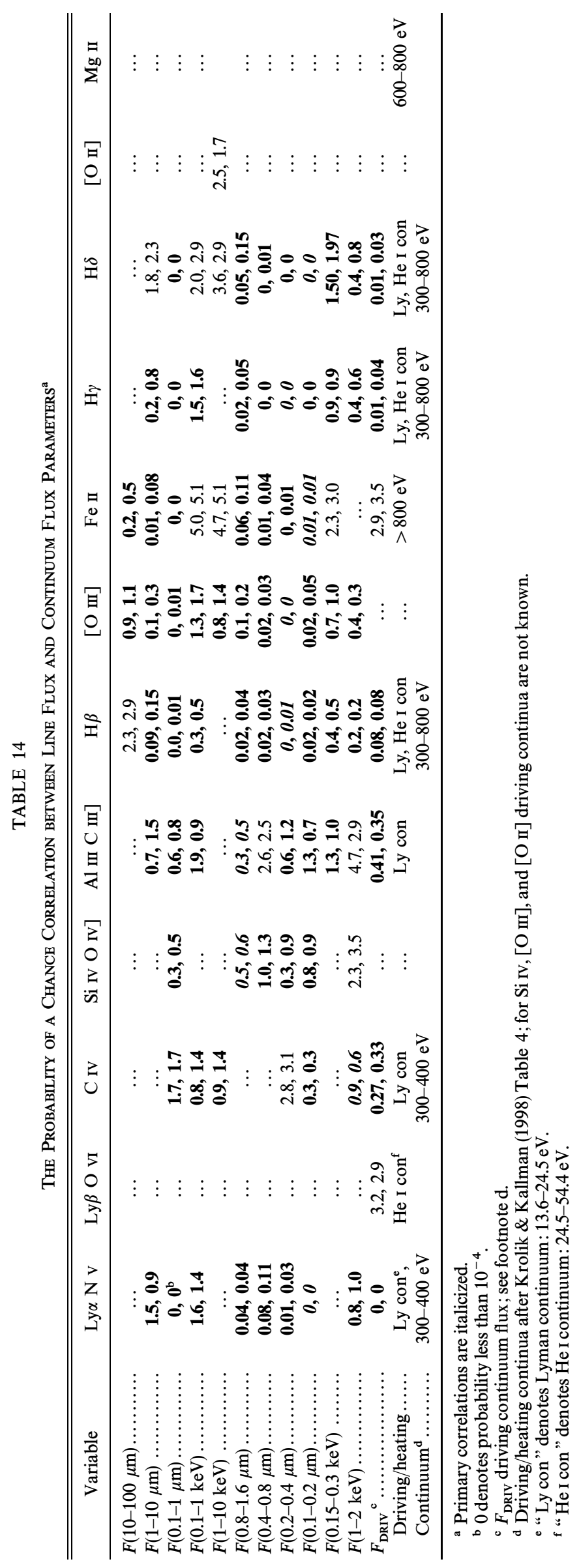


TABLE 15

CORRELATIONS

\begin{tabular}{|c|c|c|c|}
\hline Variables & Number of Correlations Tested & $\begin{array}{c}\text { Number of Random, Significant } \\
\text { Correlations Expected }\end{array}$ & $\begin{array}{l}\text { Number of Significant } \\
\text { Correlations Found }\end{array}$ \\
\hline EW vs. continuum parameters $\ldots \ldots \ldots \ldots$ & 315 & 6 & 31 \\
\hline FWHM vs. continuum parameters...... & 315 & 6 & 2 \\
\hline EW vs. line EW ......................... & 105 & 2 & 8 \\
\hline FWHM vs. line FWHM ............... & 105 & 2 & 8 \\
\hline EW vs. FWHM of same line ............ & 15 & 0.3 & 1 \\
\hline Line flux vs. continuum flux ............ & 131 & 3 & 68 \\
\hline Line flux vs. driving continuum .......... & 8 & 0.2 & 6 \\
\hline Sum & 995 & 20 & 124 \\
\hline
\end{tabular}

becomes significant after excluding the low-luminosity narrow-line AGNs (probably NLSy1; see § 3.1.1). A similar relation for $\mathrm{C}$ IV (the traditional Baldwin effect) also appears when these NLSy1s are omitted. We see a strong correlation between $\mathrm{EW}$ and $\alpha_{\mathrm{ox}}$ for $\mathrm{C}$ IV only and less significant relations for $\mathrm{Ly} \alpha, \mathrm{H} \beta$, and probably for $\mathrm{O}$ VI (although we do not have enough data to draw strong conclusions here). None of the lines correlate with X-ray luminosity, agreeing with the model predictions.

To make a more quantitative comparison between the
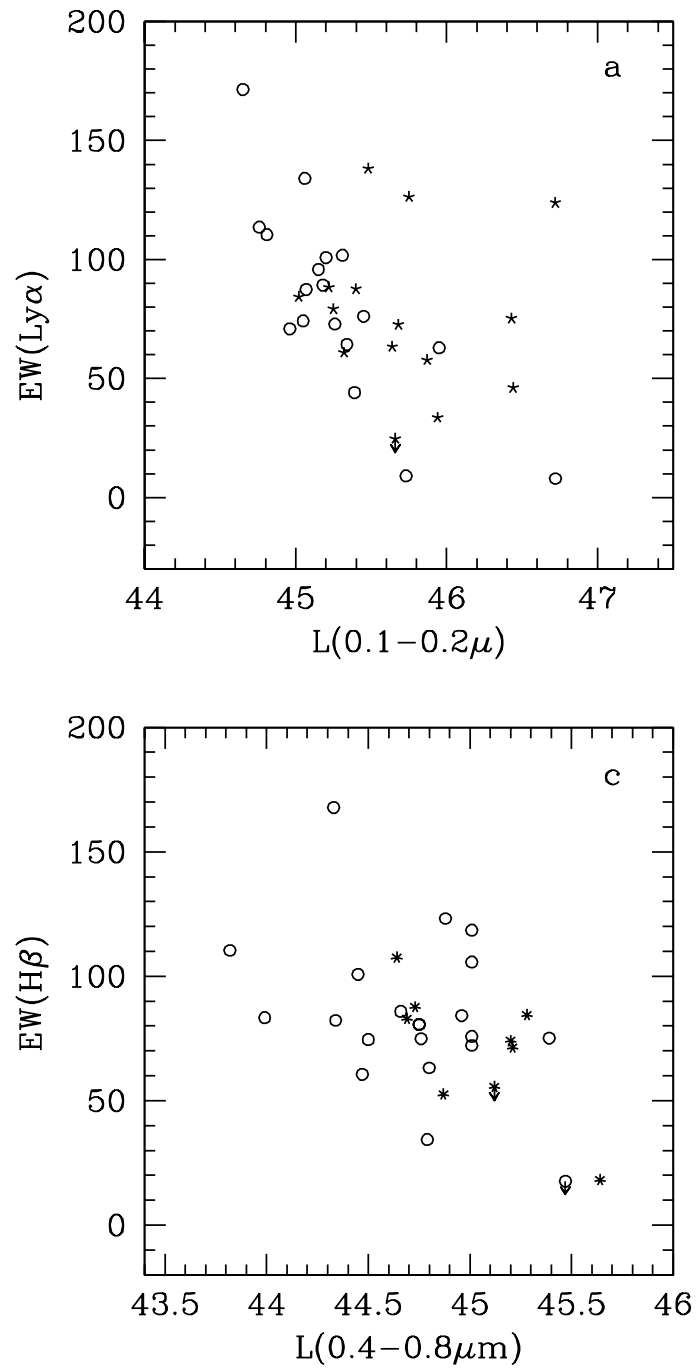

model and our data, we assume that the AD and BELR are hidden by a dusty torus similar to that postulated for Sy1 and Sy2 galaxies. Adopting an opening angle of $\sim 60^{\circ}$ (Phillips, Charles, \& Baldwin 1983) and following Netzer et al. (1992), the AD model predicts that the range in disk inclination yields a range in OUV luminosity of a factor of 2 $(0.3$ in $\log$ ). This modifies the slope $\alpha$ of the line luminosity versus driving continuum correlations in the following way:

$$
\alpha=1-0.3 /\left(\log F_{\text {cont }}^{\max }-\log F_{\text {cont }}^{\min }\right) .
$$
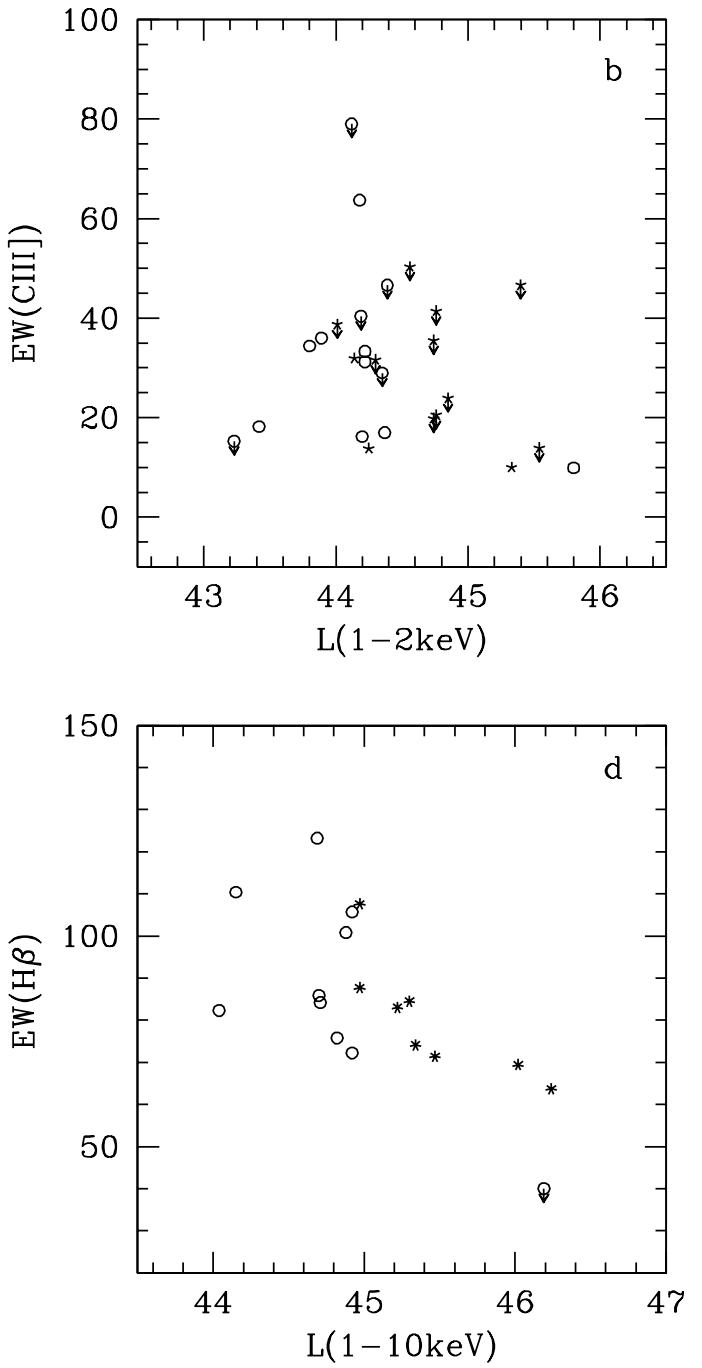

FIG. 4.-Primary correlations for UV and optical line EWs vs. continuum luminosities. RLQs are indicated by stars and RQQs by circles. 

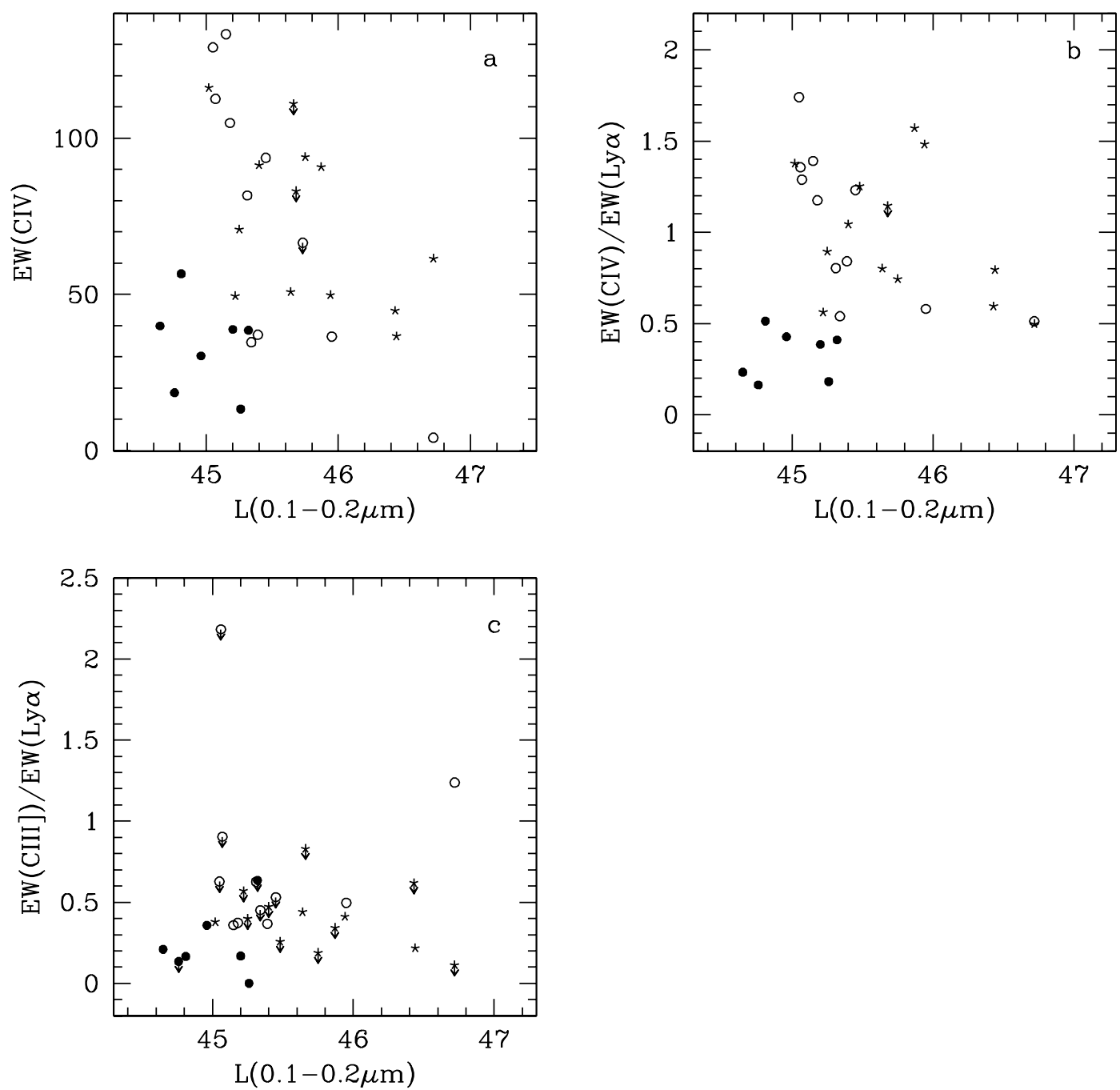

FIG. 5.-(a) Lack of Baldwin effect for EW(C Iv). (b) C IV/Ly $\alpha$ and $(c) \mathrm{C} \mathrm{III]/Ly} \alpha$ vs. UV luminosity relations. Filled circles denote NLSy1s, which decrease the significance of the $\mathrm{C}$ IV Baldwin effect in our sample.

The range in flux continuum $F_{\text {cont }}$ in our sample is $\sim 2.5$ orders of magnitude, so the expected slope is $\sim 1-0.12=$ 0.88. Most of the lines fit into this scenario $(\mathrm{Ly} \alpha, \mathrm{H} \gamma$, and probably $\mathrm{H} \beta, \mathrm{H} \delta, \mathrm{Fe}$ II, $\mathrm{O}$ VI, although the errors are large;

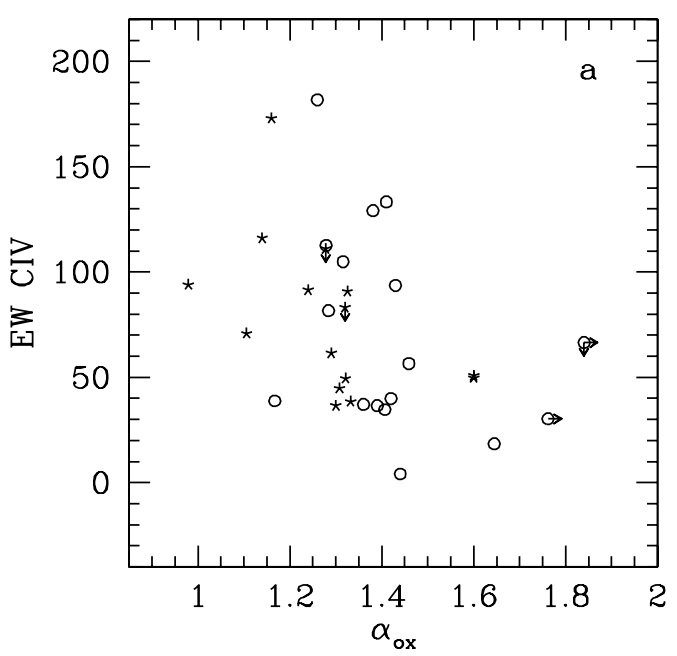

see Table 16). However, $\mathrm{C}$ IV and $\mathrm{C} \mathrm{III]} \mathrm{have} \mathrm{flatter} \mathrm{slopes}$ $(0.57 \pm 0.14$ for $\mathrm{C}$ IV and $0.69 \pm 0.19$ for $\mathrm{C}$ III]). Exclusion of the seven discrepant NLSy1 objects (see § 3.1.1) yields slopes still not steep enough $(0.64 \pm 0.14$ for C IV and $0.64 \pm 0.25$

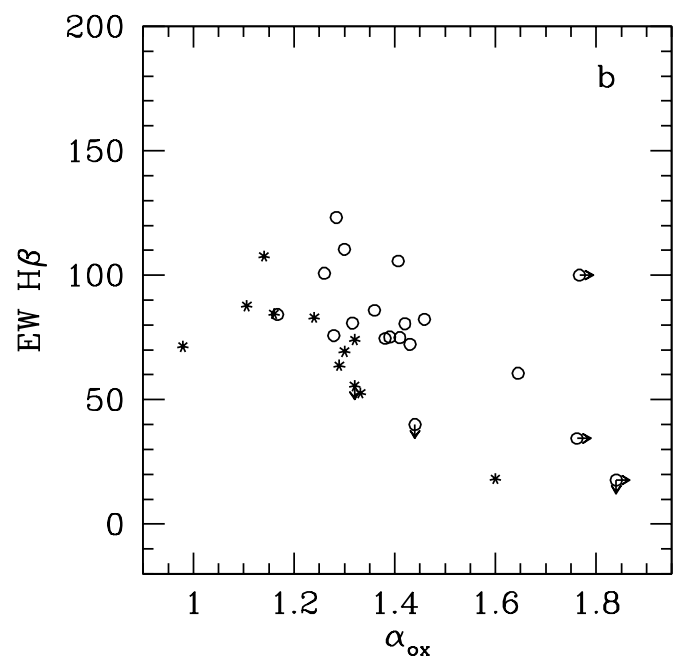

FIG. 6.-(a) $\mathrm{EW}\left(\mathrm{C}\right.$ Iv) vs. $\alpha_{\mathrm{ox}}$ correlation. (b) $\mathrm{EW}(\mathrm{H} \beta)$ vs. $\alpha_{\mathrm{ox}}$ relation. Notice quasar $1001+054$ in the upper right corner, which drastically spoils this correlation. 

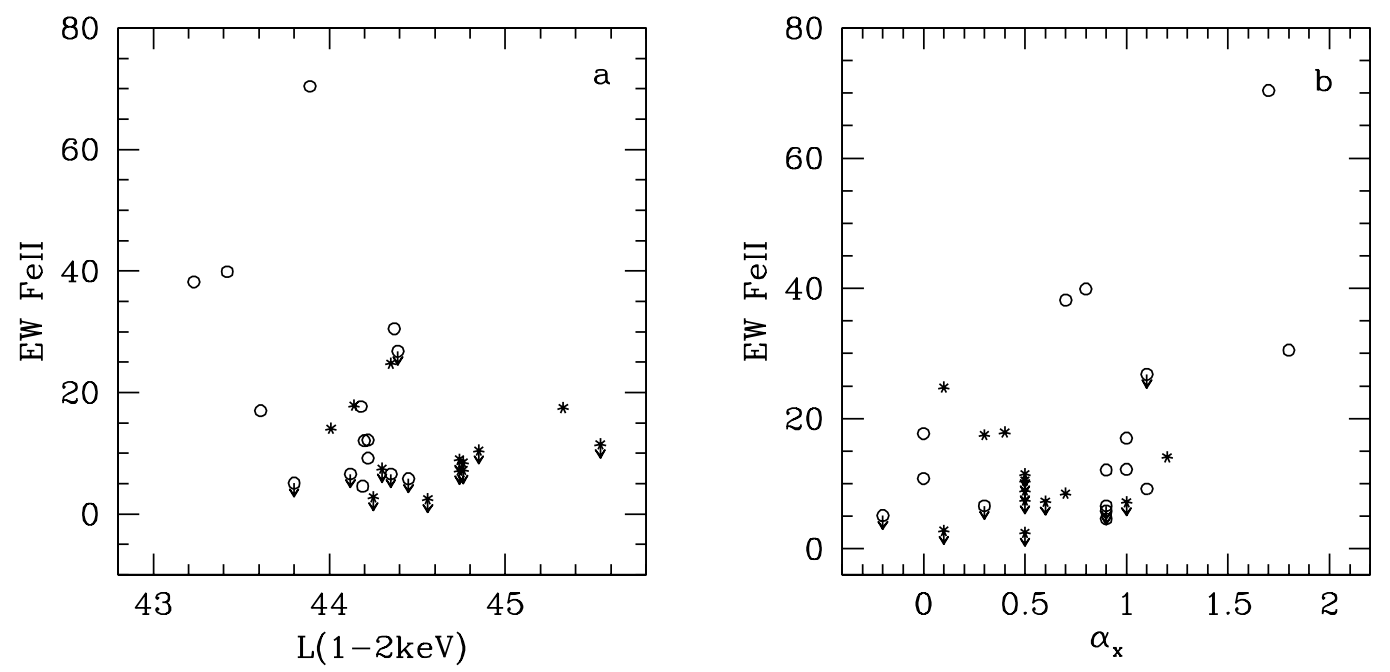

FIG. 7.- (a) Relation between the EW(Fe II) and the soft X-ray luminosity. (b) The relation between $\mathrm{EW}\left(\mathrm{Fe}\right.$ II) and $\alpha_{\mathrm{x}}$. We see no correlations but rather zones of avoidance such that objects with stronger X-ray luminosities and/or flat spectra do not have strong Fe II.
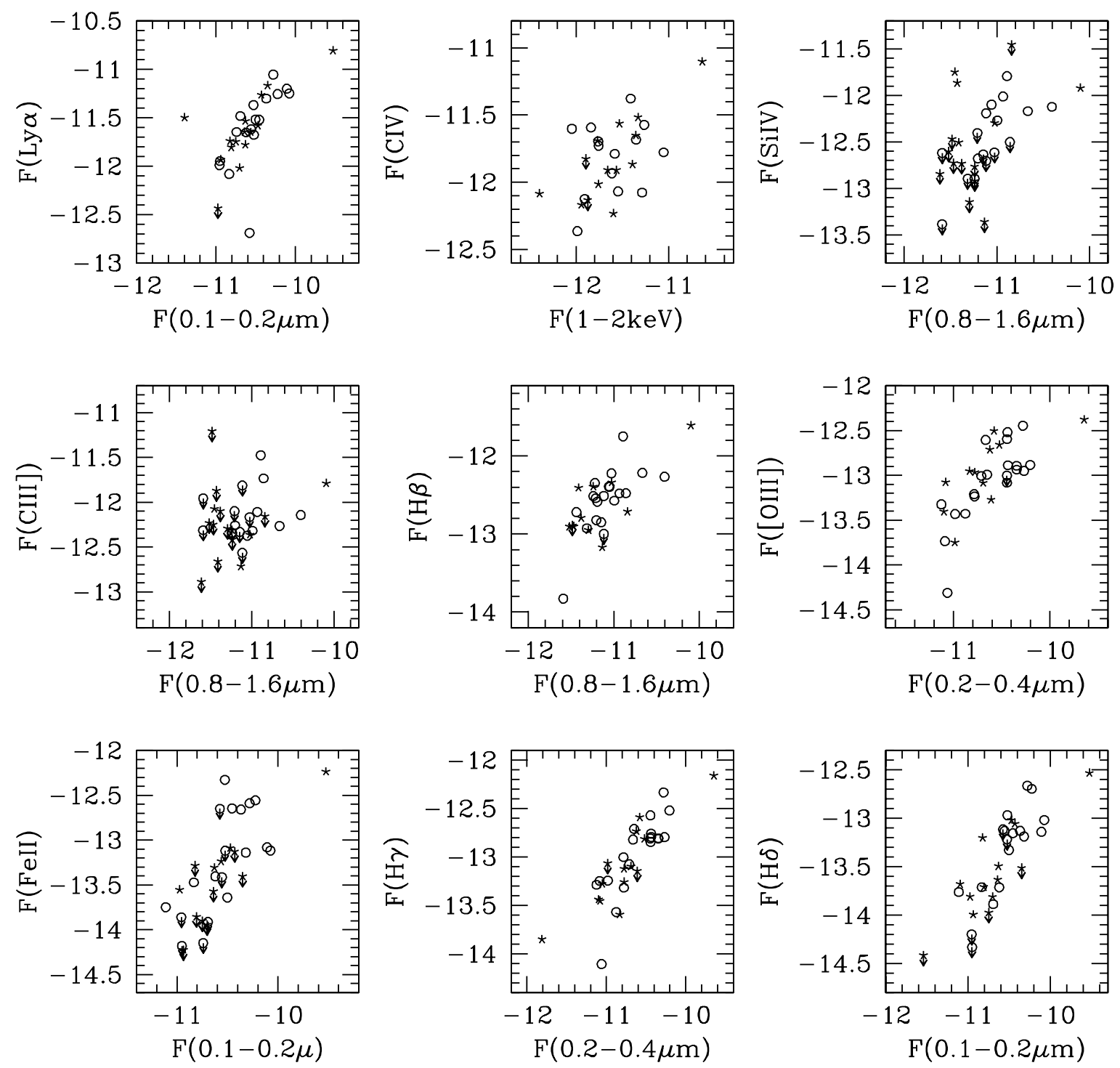

FIG. 8.-Primary correlations for the line vs. continuum fluxes 


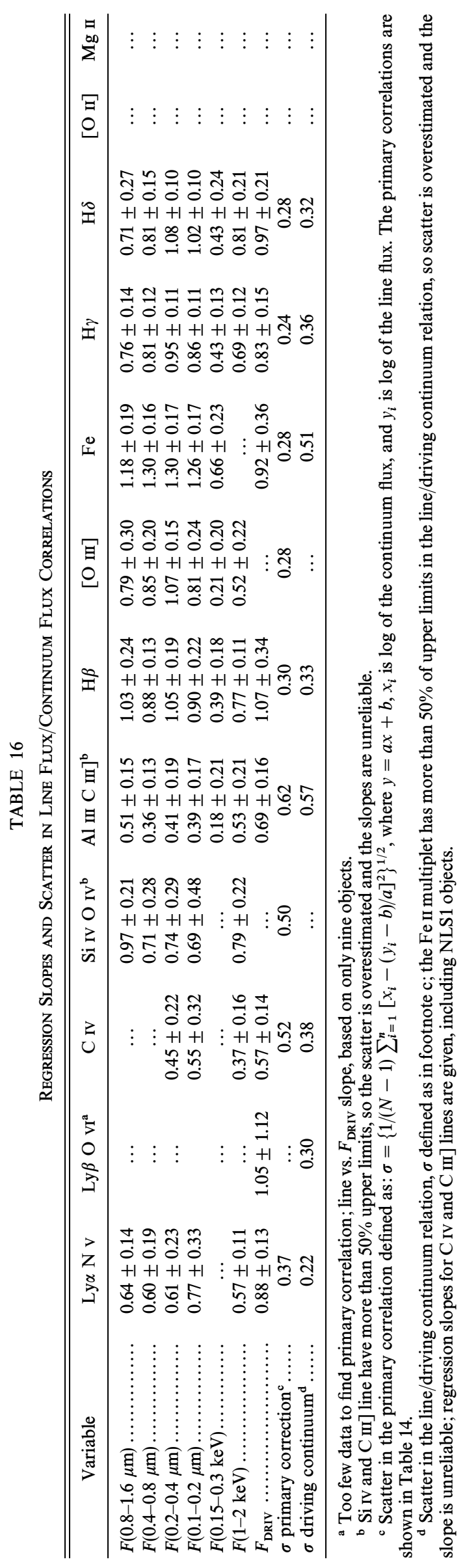



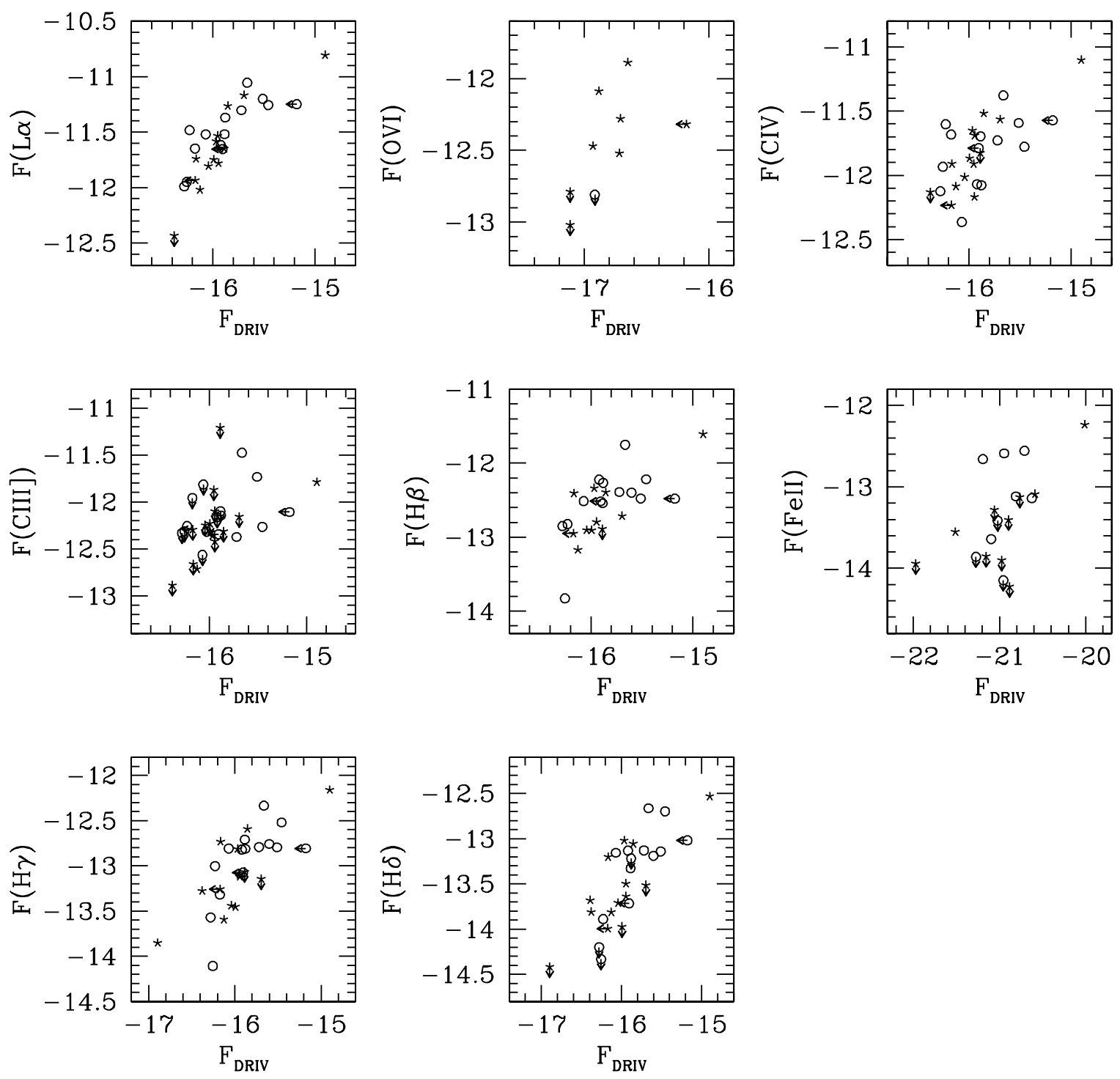

FIG. 9.-Correlations for UV and optical line fluxes vs. driving continuum fluxes

for $\mathrm{C}$ III]). (We note that the $\mathrm{C}$ III] line sample has greater than $50 \%$ upper limits, so the errors in the regression slopes are probably underestimated.) This may be due to a significant contribution from collisional excitation, which is not directly related to the ionizing continuum. In contrast, the $\mathrm{Fe}$ II line flux versus continuum flux correlations are too steep $(\sim 1.3$; see Table 16$)$ to fit this scenario. One possible explanation for this behavior is reddening by dust, which

TABLE 17

Summary OF CORRelations BetWeEn CONTINUUM PaRAMETERS

\begin{tabular}{ccccc}
\hline \hline $\log y$ & $\log x$ & $\alpha_{1}\left(y=x^{\alpha_{1}}\right)$ & $\alpha_{2}\left(x=y^{\alpha_{2}}\right)$ & $1 / \alpha_{2}$ \\
\hline$L(10-100 \mu \mathrm{m}) \ldots \ldots$ & $L(1-10 \mu \mathrm{m})$ & $1.01 \pm 0.06$ & $0.73 \pm 0.06$ & 1.37 \\
$L(10-100 \mu \mathrm{m}) \ldots \ldots$ & $L(0.1-1 \mu \mathrm{m})$ & $0.70 \pm 0.10$ & $0.71 \pm 0.20$ & 1.41 \\
$L(1-10 \mu \mathrm{m}) \ldots \ldots .$. & $L(0.1-1 \mu \mathrm{m})$ & $0.73 \pm 0.08$ & $0.97 \pm 0.13$ & 1.03 \\
$L(0.8-1.6 \mu \mathrm{m}) \ldots \ldots$. & $L(0.4-0.8 \mu \mathrm{m})$ & $0.95 \pm 0.07$ & $0.92 \pm 0.06$ & 1.07 \\
$L(0.4-0.8 \mu \mathrm{m}) \ldots \ldots$ & $L(0.2-0.4 \mu \mathrm{m})$ & $0.92 \pm 0.05$ & $0.94 \pm 0.07$ & 1.06 \\
$L(0.2-0.4 \mu \mathrm{m}) \ldots \ldots$ & $L(0.1-0.2 \mu \mathrm{m})$ & $0.88 \pm 0.02$ & $1.06 \pm 0.04$ & 0.94 \\
$L(0.1-0.2 \mu \mathrm{m}) \ldots \ldots$. & $L(1-2 \mathrm{keV})$ & $0.88 \pm 0.08$ & $0.84 \pm 0.03$ & 1.19 \\
$L(1-2 \mathrm{keV}) \ldots \ldots \ldots$ & $L(1-10 \mathrm{keV})$ & $1.04 \pm 0.12$ & $0.75 \pm 0.06$ & 1.33 \\
\hline
\end{tabular}

increases with increasing viewing angle. Given the predominance of $\mathrm{Fe}$ II emission from large viewing angles described above, this line would be reddened more than the continuum. This could lead to a stronger viewing-angle dependence than the continuum and so a steeper relation, as is observed.

Another discrepancy between our results and the predictions involves the scatter around the mean slope in the line versus continuum correlations, which is larger than that expected (i.e., greater than 0.15 around the mean; Table 16). Effects that could increase the scatter include a nonspherical geometry of the BLR clouds, random obscuration by dust in the NLR, or a different opening angle for the dusty torus. These possibilities are discussed in the following sections.

\subsubsection{The Two-Zone BELR}

The BLR is sometimes suggested to include two distinct regions: the HIL region (Ly $\alpha, \mathrm{C}$ IV, $\mathrm{C}$ III]) and LIL region (Balmer, Fe II, and Mg II lines). Collin-Soufrin et al. (1988) assumed, for example, that the LILs have a disk geometry, while the HILs do not. In this scenario, a random selection 

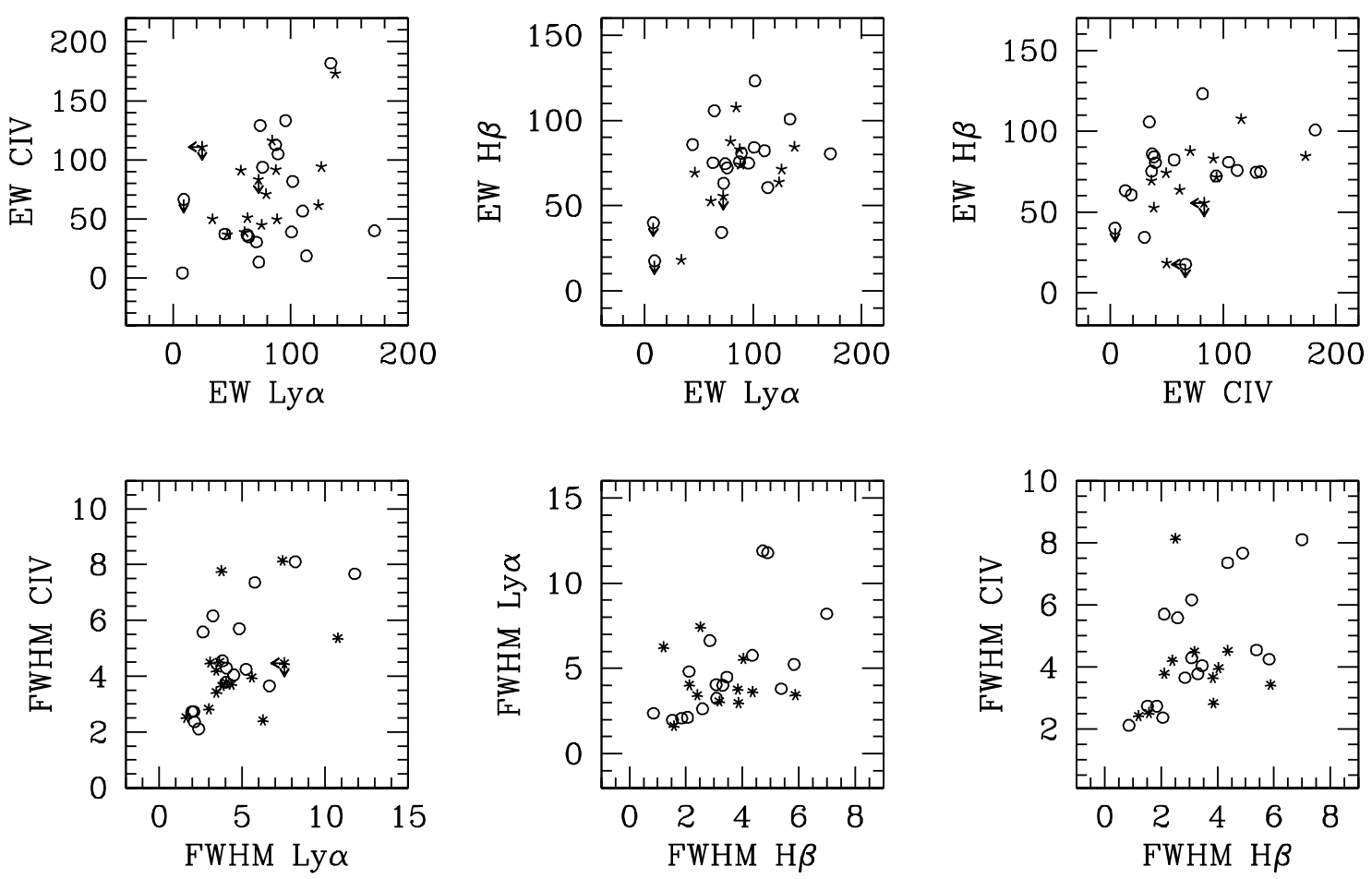

FIG. 10.-Correlations between the EW (in $\AA$ ) and FWHM (in units of $10^{-3} \mathrm{~km} \mathrm{~s}^{-1}$ ) of Ly $\alpha, \mathrm{C}$ IV, and $\mathrm{H} \beta$ lines

of objects differing only in disk inclination would lead to a scatter around the mean in a plot of $F_{\text {line }}$ versus $F_{\text {DRIV }}$ that is larger and a slope that is flatter for the HILs (as these lines have a spherical symmetry) than for the LILs. In our sample the mean slopes for the LIL Balmer and Fe II lines are indeed steeper than those for the HILs (i.e. Ly $\alpha, \mathrm{C}$ IV, and $\mathrm{C} \mathrm{III]}$; see Table 16). However, the scatter in the correlations is larger for LILs than HILs, which is contrary to the predictions. This scenario potentially offers an explana-

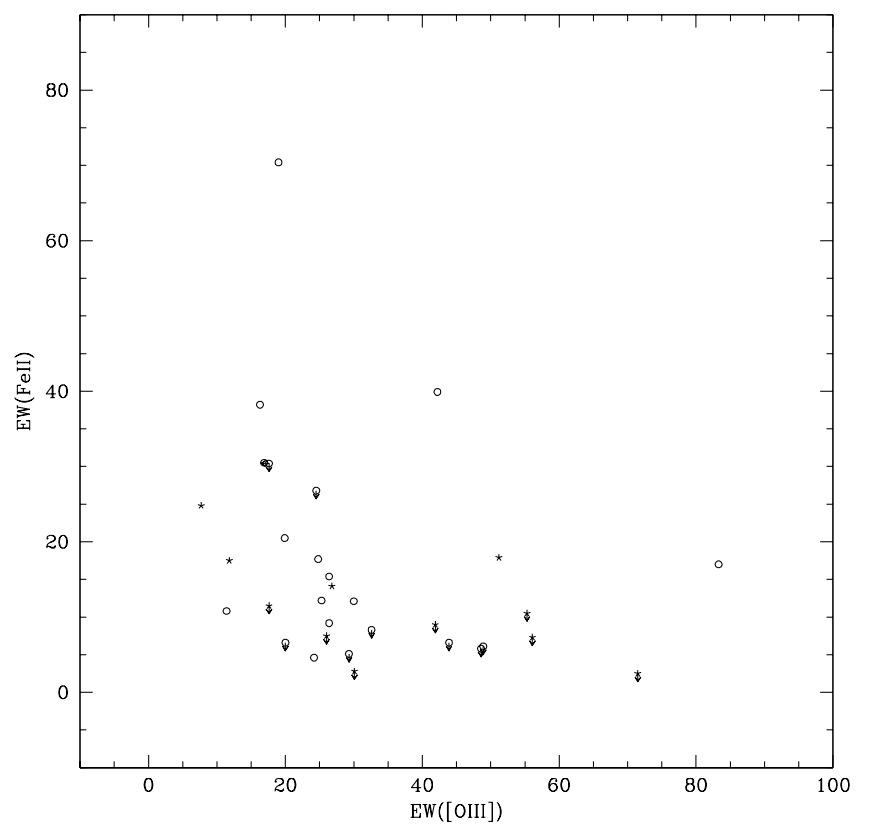

FIG. 11.-Correlation between [O III] and optical Fe II EWs (the first Boroson \& Green eigenvector). tion for the steepness of the Fe II versus OUV flux correlations if distributions of $\mathrm{Fe}$ II and OUV continuum emission from the AD differ. However, given the partial agreement between model predictions and the observations and the large uncertainties in the derived slopes, we are unable to draw strong conclusions on the applicability of this model.

\subsubsection{The Role of Dust}

The presence of dust in quasars has been discussed by many authors (e.g., Webster et al. 1995; Rawlings et al. 1995; Sprayberry \& Foltz 1992; Low et al. 1989; McLeod \& Rieke 1994a, 1994b). Baker (1997), for example, studied a complete sample of RLQs and found correlations between optical continuum and emission lines and the orientation indicator $R$ (the ratio of radio-core to lobe flux density). These correlations, and the result that core-dominated quasars are 3-5 mag brighter in the optical region than lobe-dominated quasars, were explained as due to viewingangle-dependent dust extinction. In addition, strong correlations of the EWs of [O II] and $\mathrm{Mg}$ II and the [O II $] /[\mathrm{O}$ III] ratio with $R$, the lack of significant trends for $\mathrm{C}$ III], C IV, $\mathrm{H} \beta$, and a weaker trend for [O III] suggest that the BLR and the inner part of the NLR suffer extinction similar to the continuum. The Balmer decrements $(\mathrm{H} \alpha / \mathrm{H} \beta$ and $\mathrm{H} \beta / \mathrm{H} \gamma)$ also correlate with $R$, although for $\mathrm{H} \beta / \mathrm{H} \gamma$ the correlation is less significant, probably because of contamination of $\mathrm{H} \gamma$ by [O III] $\lambda 4363$. Given the lack of measured $R$ values for the quasars in our sample, we can provide only weak, direct support for this scenario by confirming the lack of any relation between the Balmer decrement, $\mathrm{H} \beta / \mathrm{H} \gamma$, and the EW of the broad emission lines expected from the above correlations with $\mathrm{R}$.

Puchnarewicz et al. (1996), studying a sample of X-rayselected Sy1 galaxies and quasars, covering a wide range in 


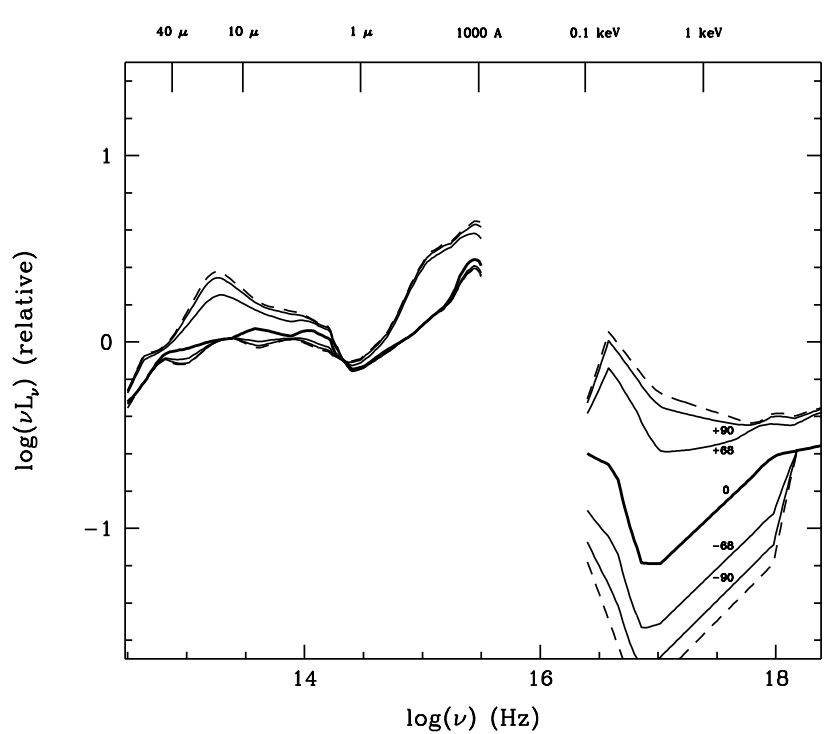

FIG. $12 a$

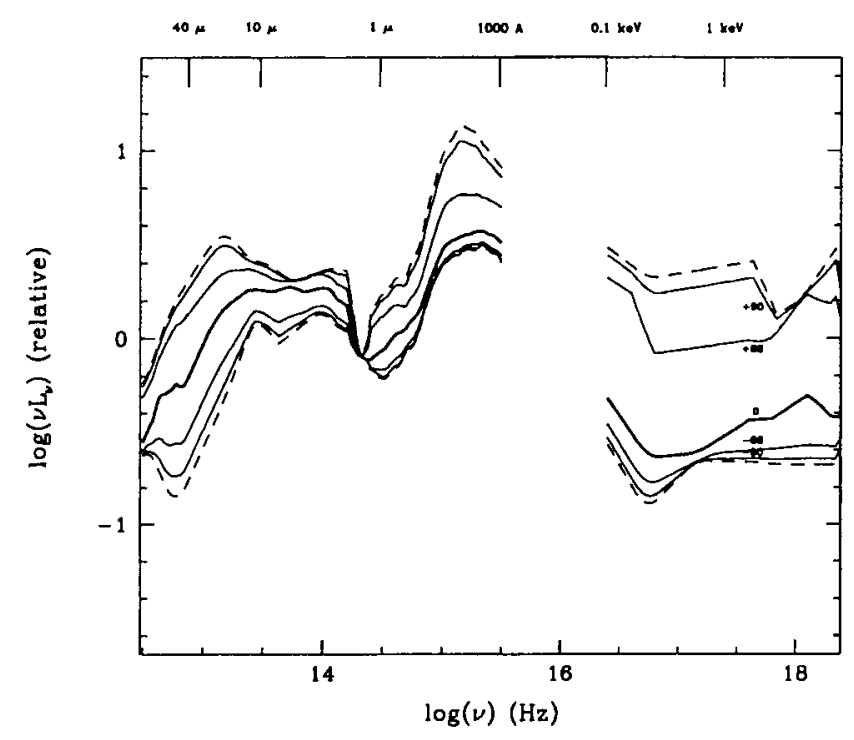

FIG. $12 b$

FIG. 12.-(a) Mean energy distributions for high-luminosity quasars, normalized at $1.5 \mu \mathrm{m}$ and the 68, 90, and 100 (dashed line) Kaplan-Meier percentile envelopes. (b) Similar mean energy distributions for lowluminosity quasars.

redshift and X-ray spectral slope, reported that the opticalto-X-ray spectra change from convex to concave as the X-ray slope, $\alpha_{x}$, hardens (i.e., $\alpha_{x}$ and $\alpha_{o x}$ anticorrelate). This was interpreted in terms of an intrinsic convex spectrum being absorbed by differing amounts of cold gas and dust, possibly depending on viewing angle and so similar to the Baker (1997) scenario. Given the small range of $\alpha_{\mathrm{x}}$ in our sample ( -0.3 to 0.9 ; see -2 to 3 for Puchnarewicz et al. 1996) and the large scatter in the correlation, we do not see a significant correlation here. However, dividing our sample according to $\alpha_{\mathrm{x}}$ and applying the median test reveals a significant trend (greater than $99 \%$ ) for $\alpha_{\text {ox }}$ to be smaller when $\alpha_{x}$ is hard. We do not find a significant correlation between $\alpha_{\text {opt }}$ (i.e., $\alpha_{\text {ouv }}$ in our sample) and $\alpha_{x}$; again, this is not surprising given our small range of $\alpha_{\mathrm{x}}$. We also note that Puchnarewicz et al. (1996) show a weak correlation for the high-redshift objects only $\left(z>1.0, P_{S}=4 \%\right)$ : their lower redshift objects do not show a correlation, which is consistent with our sample.

There are a number of other properties of our sample that could potentially be explained by dust reddening. These include the larger-than-canonical "case B" Balmer decrements $2.85<\mathrm{H} \alpha / \mathrm{H} \beta<7$ (case $\mathrm{B} \mathrm{H} \alpha / \mathrm{H} \beta=2.85$ ) and $2.1<\mathrm{H} \beta / \mathrm{H} \gamma<5.3$ (case B $\mathrm{H} \beta / \mathrm{H} \gamma=2.1$ ), implying a reddening of $A_{v}<2.04 \mathrm{mag}$; the existence of primary correlations for optical and UV lines that are not with the driving continuum (see $\S 4.1 .3$ ); the increased scatter in the line luminosity versus driving continuum relations above that predicted by the disk model; and the larger scatter within the line/continuum correlation of $\mathrm{H} \gamma$ compared with $\mathrm{H} \beta$ (see Table 16). We investigate dust as a contributor to the correlations in detail in the next section.

\subsubsection{Simulations of the Effects of Reddening}

We investigate the effects of dust on the results of our analysis by applying random amounts of dust reddening to a simulated sample of quasars with properties similar to our actual sample. First we selected the quasar in our sample with the largest $\mathrm{BBB}$, i.e., the one with largest difference: $L(0.1-0.2 \mu \mathrm{m})-L(0.8-1.6 \mu \mathrm{m})($ PG $1426+015)$. Since this object also has a ratio $\mathrm{H} \beta / \mathrm{H} \gamma$ equal to the unreddened "case B" value (2.1), we assume it is unreddened. A hypothetical sample of 26 objects was then constructed with the continuum shape of PG $1426+015$ but covering the range in bolometric luminosity present in the current sample [2.5 in $L(0.8-1.6 \mu \mathrm{m})]$, spaced by 0.1 in $\log v L_{v}$. The maximum reddening was determined from the difference in strength of the strongest and weakest BBBs in the real sample. Assuming that the dust lies outside the BELR, a random amount of reddening up to the maximum was applied to both the SED and the two extreme emission lines (in $\lambda$ ), Ly $\alpha$ and $\mathrm{H} \beta$. The process was performed twice, using two extinction curves: the standard Galactic extinction curve (Seaton 1979) and the Small Magellanic Cloud (SMC) extinction curve (Prévot et al. 1984), which corresponds well to the extinction by dust dominated by amorphous carbon grains (Czerny et al. 1995). The estimated maximum reddening was $E(B-V)=0.32$ for the Seaton extinction curve and $E(B-V)=0.20$ for the SMC extinction curve. We note that this is much less than that estimated from the Balmer decrement, implying a different amount of obscuration. We then performed a correlation analysis similar to that applied to the real sample to look for the primary correlations between $\mathrm{Ly} \alpha$ and $\mathrm{H} \beta$ and various parts of the SED and to estimate the scatter in the resulting line versus driving continuum correlations. The results are as follows: for the Seaton extinction curve the primary correlation for $L y \alpha$ was with the driving continuum and for $\mathrm{H} \beta$ with $L(0.2-0.4 \mu \mathrm{m})$, the scatter for the line versus driving continuum correlation for Ly $\alpha$ was $\sigma=0.12$ and for $\mathrm{H} \beta \sigma=0.42$; for the SMC extinction curve the primary correlation for $\mathrm{Ly} \alpha$ was with $L(0.1-$ $0.2 \mu \mathrm{m})$ and for $\mathrm{H} \beta$ was with $L(0.2-0.4 \mu \mathrm{m})$, the line versus driving continuum correlation scatter for $\operatorname{Ly} \alpha$ was $\sigma=0.12$ and for $\mathrm{H} \beta \sigma=0.20$.

A comparison of these results with the current sample (Tables 14 and 16) reveals that the simulation using the SMC extinction curve has the same primary correlations for $\mathrm{Ly} \alpha$ and $\mathrm{H} \beta$ lines as the real sample, and the scatter in the line versus driving continuum correlations is comparable with the additional scatter required to bring the results into agreement with the AD model (see Table 16). The scatter 
introduced by the Netzer AD model (§ 4.1.3) for both Ly $\alpha$ and $\mathrm{H} \beta$ is $\sigma=0.15$. The addition of dust yields an overall scatter, $\sigma \sim 0.27$ for $\operatorname{Ly} \alpha$ and $\sigma \sim 0.35$ for $\mathrm{H} \beta$, which is slightly larger than in the real sample (compare with $\sigma=0.22$ and $\sigma=0.33$, respectively). However, this slightly larger scatter is probably due to the simplifying assumption that the difference between objects with larger $\mathrm{BBB}$ and those with smaller BBB is entirely due to dust, neglecting any component due to the viewing angle of the accretion disk. Taking this into account would reduce the maximum $E(B-V)$ and improve agreement with the observations.

We conclude that the Netzer AD disk model surrounded by an optically thick dusty torus, with the addition of small amounts of dust outside the BELR, fits well into our linecontinuum correlations. Our conclusion that the SMC dust extinction curve, which corresponds well to extinction by dust dominated by small amorphous carbon grains (see Czerny et al. 1995), fits better than the Seaton extinction curve is consistent with previous results that the dust in Seyfert galaxies and quasars may differ from the dust in our Galaxy, showing a depletion of silicates (Czerny, Loska, \& Szczerba 1991; Laor \& Draine 1993).

\subsubsection{The Torus}

The assumption that quasars have the same dusty torus as the Sy1/Sy2 galaxies ( $\S 4.1 .3)$ may not be valid. Perhaps the opening angle and radius or thickness of a torus depends on the luminosity of the central engine. More luminous quasars would then have larger opening angles and larger radius/thinner tori. A large torus opening angle would naturally explain the flat slope and large scatter for the carbon $\mathrm{C}$ IV and $\mathrm{C}$ III] line versus driving continuum correlations. This scenario could also explain why we see the Sy1/Sy2 dichotomy, when there is nothing similar for quasars. However, the other lines would then be too steep for the AD model, so this scenario would require a different emitting region for the carbon lines. This seems contrived and an unlikely possibility given the generally similar behavior of the UV lines.

\subsubsection{LOC Model}

As was mentioned earlier, the majority of existing BELR models have been based on one or two zones of clouds. In recent papers, Baldwin et al. (1995) and Korista et al. (1997) model the BELR from many clouds covering a wide range in distance $r$ from the ionizing source and with a wide range in gas density $n_{\mathrm{H}}$ at each $r$. The integrated BELR spectrum is determined by integrating over the full distribution function of clouds at all radii and densities. This is called the locally optimally emitting cloud (LOC) model. This model predicts that the integrated spectrum depends only weakly on the shape of the ionizing spectrum, the column density of the clouds, and the cloud distribution with $r$ (see Fig. 2 in Baldwin 1997). This implies that the BELR spectrum is determined more by selection effects than by details of the cloud properties. Since we have concluded that the behavior of the emission lines in our sample is not purely due to photoionization effects, it seems likely that a combination of a BELR based on the LOC model, an AD providing the ionizing continuum, and a distribution of dust external to both AD and BELR would be able to fully describe our sample.

\section{CONCLUSIONS}

We investigate the relations between various emissionline parameters and continuum parameters in a sample of low-redshift $(z<1)$ quasars. The sample consists of radioloud and radio-quiet objects for which IR to soft-X-ray SEDs are available and is biased toward AGNs with strong $\mathrm{X}$-ray emission relative to the optical. Eleven newly compiled SEDs and optical spectra for all the quasars are presented. We have measured all the emission-line parameters uniformly to minimize the scatter generally introduced when combining data sets from different techniques and different authors. We use survival analysis that allows for the presence of upper limits.

We find anticorrelations between the line EWs and UV luminosities, i.e., the Baldwin effect, for the $\operatorname{Ly} \alpha$ and $\mathrm{H} \beta$ lines. Exclusion of narrow-line, low-luminosity AGNs reveals the Baldwin effect also for the $\mathrm{C}$ IV and $\mathrm{C}$ III] lines. This suggests that NLSy1 objects may have systematically lower carbon EW. We also find a significant correlation between C IV and $\alpha_{\text {ox }}$.

No correlations between the EW and the X-ray luminosity or slope are reported. However, Fe II $\lambda 4750$ shows a tendency for objects with flat X-ray spectra and/or strong $\mathrm{X}$-ray luminosities to have weak Fe II (which is consistent with Peterson et al. 1981; Phillips 1977; Osterbrock 1977). The anticorrelation between the $\mathrm{EW}([\mathrm{O} \mathrm{III}])$ and $\mathrm{EW}(\mathrm{Fe}$ II) (i.e., the first Boroson \& Green 1992 eigenvector) is present in our sample.

Correlations between the various parameters for each line were also studied. We confirm the correlations between the FWHMs and EWs of $\mathrm{C}$ IV, $\mathrm{Ly} \alpha$, and $\mathrm{H} \beta$ lines previously found by Corbin (1991) and Corbin \& Boroson (1996), indicating that the HIL and LIL components in the BLR scale similarly with luminosity. This is consistent with both a model where LILs and HILs are formed in the same clouds but in zones of differing ionization, as well as a model where LILs are formed in the atmosphere of an AD, while HILs form in a distinct spherical component.

The continuum-continuum correlations reveal that the peak of the BBB strengthens and perhaps shifts from the EUV into the UV as the luminosity increases. We confirm that the X-rays are systematically lower relative to the optical for higher luminosity objects (Wilkes et al. 1994). However, both high- and low-luminosity objects show a broad range in continuum shapes, and the distributions of SEDs for these subsamples overlap.

We investigate correlations between the line flux and various continuum fluxes, including the driving continuum flux, i.e., the part of the continuum responsible for ionizing each line. While the line-driving continuum correlations are strong, in no case were they the primary line versus continuum correlations. In a pure photoionization scenario this is surprising but can be explained by the presence of dust that reddens both the lines and continuum similarly.

All the above correlations fit into the accretion-disk model of Netzer et al. (1992 and references therein), where an optically thick, geometrically thin $\mathrm{AD}$ is composed of two components: a viewing-angle-dependent UV continuum, due to limb darkening and the change in projected surface area, and a viewing-angle-independent X-ray continuum. The model is consistent with our data, as both the predicted EW anticorrelations with UV luminosity and $\alpha_{o x}$ and the lack of correlations with the X-ray continuum are seen in our sample. A quantitative look at the line flux versus continuum flux correlations (i.e., the primary correlations, regression slopes, and scatter) reveals discrepancies that can be explained if dust is present, reddening both the 
lines and the continuum. Simulations suggest that the composition of the dust matches that of the SMC rather than our Galaxy, i.e., showing a depletion of silicates (consistent with previous studies: Czerny et al. 1995; Laor \& Draine 1993). The slopes of the line flux versus driving continuum flux correlations for $\mathrm{C}$ IV and $\mathrm{C}$ III] lines are flatter than predicted and with too large a scatter to fit into the AD plus dust model. This can be explained if, as is typical, there is a significant contribution from collisional excitation. The correlation would then be flatter, as only a part of the line flux would be directly related to the ionizing continuum.

We are grateful to Adam Dobrzycki for providing us with the HST data, Kim McLeod for precise estimates of star- light for a few objects in our sample, and Ken Lanzetta for the IUE Atlas of AGN spectra. We thank Bożena Czerny and Martin Elvis for valuable discussions and the referee for comments that improved the paper. B. J. W., J. C. M., and P. J. G. gratefully acknowledge support provided by NASA through contract NAS8-39073 (ASC). J. K. acknowledges the support of a Smithsonian predoctoral fellowship at Harvard-Smithsonian Center for Astrophysics and KBN grant 2P03D00410. S. M. acknowledges support by NASA grant NAG5-3249 (LTSA) and P. J. G. grant HF-1032.0192A awarded by the Space Telescope Science Institute, which is operated by the Association of Universities for Research in Astronomy, Inc., under NASA contract NAS526555 .
Baker, J. 1997, MNRAS, 286, 23

Baldwin, J. A. 1977, ApJ, 214, 679

1997, in ASP Conf. Ser. 113, IAU Colloq. 159, Emission Lines in

Active Galaxies: New Methods and Techniques, ed. B. M. Peterson, F. Z. Chang, \& A. S. Wilson (San Francisco: ASP), 80

Baldwin, J. A., Burke, W. L., Gaskell, C. M., \& Wampler, E. J. 1978, Nature, 273, 431

Baldwin, J. A., Ferland, G., Korista, K., \& Verner, D. 1995, ApJ, 455, L119

Baldwin, J. A., Wampler, E. J., \& Gaskell, C. M. 1989, ApJ, 338, 630

Bentley, M., Haves, P., Spencer, R. E., \& Stannard, D. 1975, MNRAS, 173, 93P

Boller, T., Brandt, W. N., \& Fink, H. 1996, A\&A, 305, 53

Boroson, T. A. 1989, ApJ, 343, 9

Boroson, T. A., \& Green, R. F. 1992, ApJS, 80, 109

Boyle, B. J., Jones, L. R., \& Shanks, T. 1991, MNRAS, 251, 482

Burstein, D., \& Heiles, C. 1978, ApJ, 225,40

Coleman, G. D., Wu, C. C., \& Weedman, D. W. 1980, ApJS, 43, 393

Collin-Soufrin, S., Dyson, J. E., McDowell, J. C., \& Perry, J. J. 1988, MNRAS, 232, 539

Comastri, A., Setti, G., Zamorani, G., Elvis, M., Giommi, P., Wilkes, B. J., \& McDowell, J. C. 1992, ApJ, 384, 62

Condon, J. J., O'Dell, S. L., Puschell, J. J., \& Stein, W. A. 1981, ApJ, 246, 624

Corbin, M. R. 1991, ApJ, 371, 51

Corbin, M. R., \& Boroson, T. A. 1996, ApJS, 107, 69

Corbin, M. R., \& Francis, P. J. 1994, AJ, 108, 2016

Crampton, D., Cowley, A. P., \& Hartwick, F. D. A. 1990, AJ, 100, 47

Cutri, R. M., Wiśniewski, W. Z., Rieke, G. M., \& Lebofsky, M. J. 1985, ApJ, 296,423

Czerny, B., Loska, Z., \& Szczerba, R. 1991, in Physics of Active Galactic Nuclei, ed. W. J. Duschl \& S. J. Wagner (Berlin: Springer), 198

Czerny, B., Loska, Z., Szczerba, R., Cukierska, J., \& Madejski, G. 1995, Acta Astron., 45, 623

Elvis, M., et al. 1994, ApJS, 95, 1 (E94)

Elvis, M., Green, R. F., Bechtold, J., Schmidt, M., Neugebauer, G., Soifer, B. T., Matthews, K., \& Fabbiano, G. 1986, ApJ, 310, 291

Ennis, D. J., \& Neugebauer, G. 1982, ApJ, 262, 460

Francis, P. J., Hewett, P. C., Foltz, C. B., \& Chaffee, F. H. 1992, ApJ, 398, 476

Green, P. J. 1996, ApJ, 467, 61

Green, P. J., et al. 1995, ApJ, 450, 51

Hyland, A. R., \& Allen, D. A. 1982, MNRAS, 199, 943

Isobe, T., Feigelson, E. D., \& Nelson, P. I. 1986, ApJ, 306, 490

Kellermann, K. I., Schmidt, M., Shaffer, D. B., \& Green, R. 1989, AJ, 98, 1195

Kendal, M., \& Stuart, A. 1976, The Advanced Theory of Statistics, Vol. 2 (New York: Macmillan)

Korista, K., Baldwin, J. A., Ferland, G., \& Verner, D. 1997, ApJS, 108, 401

Krolik, J. H., \& Kallman, T. R. 1988, ApJ, 324, 714

Kuhn, O. 1996, Ph.D. thesis, Harvard Univ.

Lanzetta, K. M., Turnshek, D. A., \& Sandoval, J. 1993, ApJS, 84, 109

Laor, A., \& Draine, B. T. 1993, ApJ, 402, 441

Laor, A., Fiore, F., Elvis, M., Wilkes, B. J., \& McDowell, J. C. 1997, ApJ, 477,93

Lawrence, A., Elvis, M., Wilkes, B. J., McHardy, I., \& Brandt N. 1997, MNRAS, 285, 879

Low, F. J., Cutri, R. M., Kleinmann, S. G., \& Huchra, J. P. 1989, ApJ, 340, L1

McDowell, J. C., Canizares, C., Elvis, M., Lawrence, A., Markoff, S., Mathur, S., \& Wilkes, B. J. 1995, ApJ, 450, 585

McLeod, K. K., \& Rieke, G. H. 1994a, ApJ, 431, 137 .1994b, ApJ, 420, 58

Miley, G. K., \& Hartsuijker, A. P. 1978, A\&AS, 34, 129

Murdoch, H. S. 1983, MNRAS, 202, 987

Mushotzky, R., \& Ferland, G. J. 1984, ApJ, 278, 558

Netzer, H. 1985, MNRAS, 216, 63
Netzer, H. 1987, MNRAS, 225, 55

Netzer, H., Laor, A., \& Gondhalekar, P. M. 1992, MNRAS, 254, 15

Neugebauer, G., Green, R. F., Matthews, K., Schmidt, M., Soifer, B. T., \& Bennett, J. 1987, ApJS, 63, 615

Neugebauer, G., Miley, G. K., Soifer, B. T., \& Clegg, P. E. 1986, AJ, 308, 815

Neugebauer, G., Oke, J. B., Becklin, E. E., \& Matthews, K. 1979, ApJ, 230, 79

Osmer, P. S. 1980, ApJS, 42, 523

Osterbrock, D. E. 1977, ApJ, 215, 733

Osterbrock, D. E., \& Pogge, R. W. 1985, ApJ, 297, 166

Owen, F. N., Porcas, R. W., Mufson, S. L., \& Moffett, T. J. 1978, AJ, 83, 685

Owen, F. N., \& Puschell, J. J. 1982, AJ, 87, 595

Parley, R. A. 1982, AJ, 82, 859

Penfold, J. E. 1979, MNRAS, 186, 297

Peterson, J. G., Foltz, C. B., \& Byard, P. L. 1981, ApJ, 251, 4

Phillips, M. M. 1977, ApJ, 215, 746

Phillips, M. M., Charles, P. A., \& Baldwin, J. A. 1983, ApJ, 266, 485

Pogge, R. W., \& Peterson, B. M. 1992, AJ, 103, 1084

Pooley, G. G., \& Henbest, S. N. 1974, MNRAS, 169, 477

Pounds, K. A., Done, C., \& Osborne, J. P. 1996, MNRAS, 277, 1430

Prévot, M. L., Lequeux, J., Maurice, E., Prévot, E., \& Rocca-Volmerange, B. 1984, A\&A, 132, 389

Puchnarewicz, E. M., et al. 1996, MNRAS, 281, 1243

Rawlings, S., Lacy, M., Sivia, D. S., \& Eales, S. A. 1995, MNRAS, 273, 821

Robertson, J. G. 1986, PASP, 98, 1220

Robson, E. I., Gear, W. K., Smith, M. G., Ade, P. A. R., \& Nolt, I. G. 1985, MNRAS, 213, 355

Romanishin, W., \& Hintzen, P. 1989, ApJ, 341, 41

Sanders, D. B., Phinney, E. S., Neugebauer, G., Soifer, B. T., \& Matthews, K. 1989, ApJ, 347, 29

Seaton, M. J. 1979, MNRAS, 187, 73P

Shastri, P., Wilkes, B. J., Elvis, M., \& McDowell, J. 1993, ApJ, 410, 29

Sitko, M. L., Stein, W. A., Zhang, Y.-X., \& Wiśniewski, W. Z. 1982, ApJ, 259,486

Smith, E. P., Heckman, T. M., Bothun, G. D., Romanishin, W., \& Balick, B. 1986, ApJ, 306, 64

Sprayberry, D., \& Foltz, C. B. 1992, ApJ, 390, 39

Steppe, M., Salter, C. J., Chini, R., Kreysa, E., Brunswig, W., \& Perez, J. L. 1988, A\&AS, 75, 317

Swarup, G., Sinha, R. P., \& Hilldrup, K. 1984, MNRAS, 208, 813

Tananbaum, H., Avni, Y., Green, R. F., Schmidt, M., \& Zamorani, G. 1986, ApJ, 305, 57

Taylor, G. L., Dunlop, J. S., Hughes, D. H., \& Robson, E. I. 1996, MNRAS, 283,930

Tytler, D., \& Fan, X.-M. 1992, ApJS, 79, 1

Walter, R., \& Fink, H. H. 1993, A\&A, 274, 105

Wampler, E. J., Gaskell, C. M., Burke, W. L., \& Baldwin, J. A. 1984, ApJ, 276,403

Webster, R. L., Francis, P. J., Peterson, B. A., Drinkwater, M. J., \& Masci, F. J. 1995, Nature, 375, 469

Wilkes, B. J., \& Elvis, M. 1987, ApJ, 323, 243

Wilkes, B. J., Elvis, M., \& McHardy, I. 1987, ApJ, 321, 23

Wilkes, B. J., \& McDowell, J. C. 1995, in ASP Conf. Ser. 61, Astronomical Data Analysis and Software Systems III, ed. D. Crabtree, R. Hanisch, \& J. Barnes (San Francisco: ASP), 423

Wilkes, B. J., Tananbaum, H., Worrall, D. M., Avni, Y., Oey, M. S., \& Flanagan, J. 1994, ApJS, 92, 53

Williams, O. R., et al. 1992, ApJ, 389, 157

Wills, B. J., Brotherton, M. S., Fang, D., Steidel, C. C., \& Sargent, W. L. W. 1993, ApJ, 415, 563

Zamorani, G., Marano, B., Mignoli, M., Zitelli, V., \& Boyle, B. J. 1992, MNRAS, 256, 238

Zheng, W., Kriss G. A., \& Davidsen, A. F. 1995, ApJ, 440, 606

Zheng, W., \& Malkan, M. A. 1993, ApJ, 415, 517

Zheng, W., \& O'Brien, P. T. 1990, ApJ, 353, 433 